\title{
Synthesis of 2,3-Disubstituted Benzo[b]selenophenes via Electrophilic Cyclization
}

\author{
Tanay Kesharwani, Shilpa A. Worlikar and Richard C. Larock* \\ Department of Chemistry, Iowa State University, Ames, Iowa 50011 \\ larock@iastate.edu
}

\section{Table of Contents}

Pages

General

S2-S2

Preparation of 2-iodo(methylselenyl)arenes

S2-S3

Preparation 1-(1-alkynyl)-2-(methylselenyl)arenes

Iodocyclization of 1-(alkynyl)-2-(methylselenyl)arenes

S7-S11

Bromocyclization of 1-(alkynyl)-2-(methylselenyl)arenes

S11-S12

$\mathrm{PhSeCl}$ and $\mathrm{PhSeBr}$ cyclization of 1-(alkynyl)-2-(methylselenyl)arenes

S12-S13

Derivatization of 3-iodobenzo[b]selenophenes

S13-S15

Copies of ${ }^{1} \mathrm{H}$ and ${ }^{13} \mathrm{C}$ NMR spectra

S16-S81 
General. The ${ }^{1} \mathrm{H}$ and ${ }^{13} \mathrm{C}$ NMR spectra were recorded at 300 and $75.5 \mathrm{MHz}$. Thin layer chromatography was performed using commercially prepared 60-mesh silica gel plates, and visualization was effected with short wavelength UV light $(254 \mathrm{~nm})$. All melting points are uncorrected.

Reagents. All reagents were used directly as obtained commercially unless otherwise noted.

General preparation of 2-iodo(methylselenyl)arenes. To a stirred solution of methanol (30 mL) and $10 \mathrm{mmol}$ of the 2-iodoaniline, $20 \mathrm{mmol}$ of $\mathrm{HBF}_{4}$ (36 mL, 48\% solution) was added dropwise. After the addition was complete, the solution was allowed to cool to $0{ }^{\circ} \mathrm{C}$. To this solution an aqueous solution of $\mathrm{NaNO}_{2}(12 \mathrm{mmol}$ in $5 \mathrm{~mL}$ of water) was added dropwise to the reaction mixture, which turned a pale yellow to red brown. The mixture was allowed to warm to room temperature and methanol was removed under vacuum at room temperature. The mixture was filtered and washed with cold methanol. The diazonium salt was dried under vacuum and used for the next step without purification. A suspension of $8 \mathrm{mmol}$ of the crude diazonium salt in $25 \mathrm{~mL}$ of $\mathrm{CHCl}_{3}$ containing $10 \mathrm{~mol} \%$ of 18-crown-6 and $9 \mathrm{mmol}$ of dimethyl diselenide was stirred at $0{ }^{\circ} \mathrm{C}$. To this mixture, $16 \mathrm{mmol}$ of KOAc was added in small portions over a period of $10 \mathrm{~min}$ and the resulting solution was allowed to stir for $4 \mathrm{~h}$ and then filtered. The solid residue was washed with chloroform and the resulting filtrate was washed with water ( $2 \times 5 \mathrm{~mL})$, dried over anhydrous $\mathrm{Na}_{2} \mathrm{SO}_{4}$, and concentrated under vacuum. The crude product obtained was then purified by flash chromatography on silica gel using ethyl acetate/hexanes as the eluent.

2-Iodophenyl methyl selenide (1). The product was obtained as a yellow oil: ${ }^{1} \mathrm{H}$ NMR $\left(\mathrm{CDCl}_{3}\right) \delta 2.34(\mathrm{~s}, 3 \mathrm{H}), 6.89(\mathrm{dt}, J=1.4,7.7 \mathrm{~Hz}, 1 \mathrm{H}), 7.17-7.20(\mathrm{~m}, 1 \mathrm{H})$, 7.28-7.34 (m, 2H), 7.74 
(dd, $J=1.2,7.9 \mathrm{~Hz}, 1 \mathrm{H}) ;{ }^{13} \mathrm{C}$ NMR $\left(\mathrm{CDCl}_{3}\right) \delta$ 9.1, 100.0, 126.9, 128.2, 128.8, 139.4, 139.6; IR (neat, $\mathrm{cm}^{-1}$ ) 3050, 2998, 2923, 1561, 1422; HRMS calcd for $\mathrm{C}_{7} \mathrm{H}_{7} \mathrm{ISe} 297.87575$, found 297.87617.

Methyl 3-iodo-4-(methylselenyl)benzoate (2). The product was obtained as a orange semisolid: ${ }^{1} \mathrm{H}$ NMR $\left(\mathrm{CDCl}_{3}\right) \delta 2.35$ (s, 3H), 3.89 (s, 3H), 7.15 (d, $\left.J=8.2 \mathrm{~Hz}, 1 \mathrm{H}\right), 7.91$ (dd, $J=$ 8.3, $1.8 \mathrm{~Hz}, 1 \mathrm{H}), 8.35$ (d, $J=1.7 \mathrm{~Hz}, 1 \mathrm{H}) ;{ }^{13} \mathrm{C} \mathrm{NMR}\left(\mathrm{CDCl}_{3}\right) \delta$ 9.4, 52.4, 98.3, 126.9, 128.3, 129.4, 140.0, 147.2, 165.7; IR (neat, $\mathrm{cm}^{-1}$ ) 3060, 3010, 2954, 2920, 1719, 1290; HRMS calcd for $\mathrm{C}_{9} \mathrm{H}_{9} \mathrm{IO}_{2} \mathrm{Se} 355.88125$, found 355.88200.

General procedure for the palladium/copper-catalyzed formation of 1-(1-alkynyl)-2(methylselenyl)arenes. To a solution of $\mathrm{Et}_{3} \mathrm{~N}(10 \mathrm{~mL}), 2.0 \mathrm{mmol}$ of the 2iodo(methylselenyl)arene and $\mathrm{PdCl}_{2}\left(\mathrm{PPh}_{3}\right)_{2}$ (2 mol \%) (stirring for 5 min beforehand), $\mathrm{CuI}$ (1 mol \%) was added and the flask was sealed and flushed with Ar. 3.0 Mmol of terminal acetylene dissolved in $2 \mathrm{~mL}$ of $\mathrm{Et}_{3} \mathrm{~N}$ was then added dropwise and the reaction mixture was allowed to stir at room temperature for the desired time. After the reaction was over, the resulting solution was filtered, washed with satd aq $\mathrm{NaCl}$ and extracted with diethyl ether $(3 \times 15 \mathrm{~mL})$. The combined ether fractions were dried over anhydrous $\mathrm{Na}_{2} \mathrm{SO}_{4}$ and concentrated under vacuum to yield the crude product. The crude product was purified by flash chromatography on silica gel using ethyl acetate/hexanes as the eluent.

2-(1-Decynyl)phenyl methyl selenide (3). The product was obtained as a yellow oil: ${ }^{1} \mathrm{H}$ NMR $\left(\mathrm{CDCl}_{3}\right) \delta$ 0.86-0.90 (t, $\left.J=6.9 \mathrm{~Hz}, 3 \mathrm{H}\right), 1.28-1.30(\mathrm{~m}, 8 \mathrm{H}), 1.43-1.51(\mathrm{~m}, 3 \mathrm{H}), 1.60-1.69$ 
(m, 2H), 2.31 (s, 1H), 2.45-2.49 (t, $J=6.9 \mathrm{~Hz}, 2 \mathrm{H}), 7.05-7.11$ (m, 1H), 7.16-7.21 (m, 2H), 7.32$7.35(\mathrm{~d}, J=8.2 \mathrm{~Hz}, 1 \mathrm{H}) ;{ }^{13} \mathrm{C} \mathrm{NMR}\left(\mathrm{CDCl}_{3}\right) \delta$ 6.0, 14.3, 19.8, 22.9, 28.9, 29.1, 29.3, 29.4, 32.0, 79.2, 96.9, 124.4, 125.1, 127.0, 128.3, 132.3, 136.2; IR (neat, $\mathrm{cm}^{-1}$ ) 3057, 2926, 2853, 2226, 1581, 1460; HRMS calcd for $\mathrm{C}_{17} \mathrm{H}_{24}$ Se 308.10432, found 308.10500.

2-(3-Cyclohexylprop-1-ynyl)phenyl methyl selenide (4). The product was obtained as a yellow liquid: ${ }^{1} \mathrm{H}$ NMR $\left(\mathrm{CDCl}_{3}\right)$ $\delta$ 1.05-1.35 (m, 5H), 1.56-1.78 (m, 4H), 1.89-1.93 (m, 2H), 2.32 (s, 1H), 2.37-2.39 (d, $J=6.6 \mathrm{~Hz}, 2 \mathrm{H}), 7.06-7.13$ (m, 1H), 7.17-7.22 (m, 2H), 7.33-7.36 (d, $J=$ $7.4 \mathrm{~Hz}, 1 \mathrm{H}) ;{ }^{13} \mathrm{C}$ NMR $\left(\mathrm{CDCl}_{3}\right) \delta$ 6.1, 26.4, 26.5, 27.7, 33.0, 37.7, 80.1, 95.8, 124.5, 125.2, 127.0, 128.3, 132.4, 136.2; IR (neat, $\mathrm{cm}^{-1}$ ) 3058, 2923, 2850, 2227, 1460; HRMS calcd for $\mathrm{C}_{16} \mathrm{H}_{20} \mathrm{Se} 292.07302$, found 292.07347.

6-[2-(Methylselenyl)phenyl]hex-5-ynenitrile (5). The product was obtained as a yellow oil: ${ }^{1} \mathrm{H}$ NMR $\left(\mathrm{CDCl}_{3}\right) \delta$ 1.96-2.03 (q, $\left.J=5.3 \mathrm{~Hz}, 2 \mathrm{H}\right), 2.33$ (s, 3H), 2.64-2.69 (m, 4H), 7.08-7.14 (m, $1 \mathrm{H}), 7.22-7.24(\mathrm{~m}, 2 \mathrm{H}), 7.33-7.35(\mathrm{~d}, J=5.6 \mathrm{~Hz}, 1 \mathrm{H}) ;{ }^{13} \mathrm{C} \mathrm{NMR}\left(\mathrm{CDCl}_{3}\right) \delta 6.3,16.5,19.0$, 24.9, 81.2, 93.2, 119.6, 123.5, 125.3, 127.2, 128.9, 132.4, 136.3; IR (neat, $\mathrm{cm}^{-1}$ ) 3056, 3005, 2929, 2247, 1580, 1460; HRMS calcd for $\mathrm{C}_{13} \mathrm{H}_{13} \mathrm{NSe} 263.02132$, found 263.02180.

Methyl 6-[2-(methylselenyl)phenyl]hex-5-ynoate (6). The product was obtained as a yellow oil: ${ }^{1} \mathrm{H}$ NMR $\left(\mathrm{CDCl}_{3}\right) \delta$ 1.94-2.01 (q, $\left.J=5.3 \mathrm{~Hz}, 2 \mathrm{H}\right), 2.32(\mathrm{~s}, 3 \mathrm{H}), 2.55-2.60(\mathrm{~m}, 4 \mathrm{H})$, 3.69 (s, 3H), 7.08-7.13 (m, 1H), 7.19-7.23 (m, 2H), 7.33-7.35 (d, $J=6.1 \mathrm{~Hz}, 1 \mathrm{H}) ;{ }^{13} \mathrm{C}$ NMR $\left(\mathrm{CDCl}_{3}\right) \delta$ 6.1, 19.3, 24.1, 33.1, 51.8, 80.1, 95.2, 124.0, 125.2, 127.1, 128.6, 132.4, 126.3, 173.8; 
IR (neat, $\mathrm{cm}^{-1}$ ) 3056, 2997, 2948, 2841, 2226, 1735, 1581, 1460; HRMS calcd for $\mathrm{C}_{14} \mathrm{H}_{16} \mathrm{O}_{2} \mathrm{Se}$ 296.03155, found 296.03210.

2-Methyl-4-[2-(methylselenyl)phenyl]but-3-yn-2-ol (7). The product was obtained as a brown oil: ${ }^{1} \mathrm{H}$ NMR $\left(\mathrm{CDCl}_{3}\right) \delta 1.59$ (s, 6H), 2.17 (s, 1H), 2.26 (s, 3H), 7.01-7.08 (m, 1H), 7.137.19 (m, 2H), 7.27-7.30 (m, 1H); ${ }^{13} \mathrm{C}$ NMR $\left(\mathrm{CDCl}_{3}\right)$ 86.1, 31.6, 65.9, 80.8, 99.8, 123.1, 125.3, 127.3, 129.1, 132.3, 136.7; IR (neat, $\mathrm{cm}^{-1}$ ) 3419, 2977, 2868, 1642, 1444; HRMS calcd for $\mathrm{C}_{12} \mathrm{H}_{14} \mathrm{OSe} 254.02099$, found 254.02129.

Triethyl[(2-(methylselenyl)phenyl)ethynyl]silane (8). The product was obtained as a pale yellow oil: ${ }^{1} \mathrm{H}$ NMR $\left(\mathrm{CDCl}_{3}\right) \delta$ 0.66-0.74 (q, $\left.J=8.4 \mathrm{~Hz}, 6 \mathrm{H}\right), 1.05-1.10(\mathrm{t}, J=7.7 \mathrm{~Hz}, 9 \mathrm{H}), 2.23$ (s, 3H), 7.07-7.13 (m, 1H), 7.22-7.24 (m, 2H), 7.40-7.43 (m, $1 \mathrm{H}) ;{ }^{13} \mathrm{C}$ NMR $\left(\mathrm{CDCl}_{3}\right) \delta 4.68$, 6.10, 7.82, 98.43, 104.19, 123.73, 125.11, 127.09, 129.16, 132.95, 136.91; IR (neat, $\mathrm{cm}^{-1}$ ) 3017, 2955, 2931, 2874, 2152, 1457; HRMS calcd for $\mathrm{C}_{15} \mathrm{H}_{22} \mathrm{SeSi}$ 310.06560, found 310.06610.

2-(Cyclohex-1-enylethynyl)phenyl methyl selenide (9). The product was obtained as a yellow liquid: ${ }^{1} \mathrm{H}$ NMR $\left(\mathrm{CDCl}_{3}\right) \delta$ 1.57-1.72 (m, 4H), 2.12-2.24 (m, 2H), 2.25-2.31 (m, 5H), 6.24-6.26 (m, 1H), 7.04-7.14 (m, 1H), 7.16-7.22 (m, 2H), 7.34 (d, $J=7.6 \mathrm{~Hz}, 1 \mathrm{H}) ;{ }^{13} \mathrm{C}$ NMR $\left(\mathrm{CDCl}_{3}\right)$ 5.9, 21.6, 22.4, 25.9, 29.2, 85.3, 97.2, 120.7, 124.0, 125.1, 127.0, 128.4, 131.9, 135.6, 136.2; IR (neat, $\mathrm{cm}^{-1}$ ) 3054, 2927, 2856, 2197, 1578, 1458, 1433; HRMS calcd for $\mathrm{C}_{15} \mathrm{H}_{16} \mathrm{Se}$ 276.04171, found 276.042303. 
2-(Phenylethynyl)phenyl methyl selenide (10). The product was obtained as a yellow oil: ${ }^{1} \mathrm{H}$ NMR $\left(\mathrm{CDCl}_{3}\right) \delta 2.35$ (s, 3H), 7.13-7.17 (m, 1H), 7.22-7.37 (m, 5H), 7.46-7.48 (d, $J=7.6 \mathrm{~Hz}$, 1H), 7.56-7.59 (m, 2H); ${ }^{13} \mathrm{C} \mathrm{NMR}\left(\mathrm{CDCl}_{3}\right) \delta$ 6.26, 88.01, 95.23, 123.26, 123.78, 125.37, 127.52, 128.53, 128.64, 129.02, 131.77, 132.34, 136.67; IR (neat, $\mathrm{cm}^{-1}$ ) 3061, 3016, 2929, 2399, 1598, 1490; HRMS calcd for $\mathrm{C}_{15} \mathrm{H}_{12}$ Se 272.01042, found 272.01100.

Methyl 2-[(4-nitrophenyl)ethynyl]phenyl selenide (11). The product was obtained as a orange solid: mp 99-101 ${ }^{\circ} \mathrm{C} ;{ }^{1} \mathrm{H}$ NMR $\left(\mathrm{CDCl}_{3}\right) \delta 2.39$ (s, 3H), 7.17-7.22 (m, 1H), 7.31-7.33 (m, 2H), 7.49 (d, $J=7.6 \mathrm{~Hz}, 1 \mathrm{H}), 7.69-7.73(\mathrm{~m}, 2 \mathrm{H}), 8.21-8.25(\mathrm{~m}, 2 \mathrm{H}) ;{ }^{13} \mathrm{C} \mathrm{NMR}\left(\mathrm{CDCl}_{3}\right) \delta 6.4$, 93.1, 93.2, 122.6, 123.9, 125.5, 127.7, 130.0, 130.2, 132.4, 132.7, 137.3, 147.3; IR (neat, $\mathrm{cm}^{-1}$ ) 3018, 2933, 2214, 1595, 1518; HRMS calcd for $\mathrm{C}_{15} \mathrm{H}_{11} \mathrm{NO}_{2} \mathrm{Se} 316.99549$, found 316.99602 .

2-[(2-Methoxyphenyl)ethynyl]phenyl methyl selenide (12). The product was obtained as a pale yellow solid: mp $75-77{ }^{\circ} \mathrm{C} ;{ }^{1} \mathrm{H}$ NMR $\left(\mathrm{CDCl}_{3}\right) \delta 2.37$ (s, 3H), $3.93(\mathrm{~s}, 3 \mathrm{H}), 6.89-6.97$ (m, 2H), 7.12-7.17 (m, 1H), 7.21-7.24 (m, 1H), 7.26-7.34 (m, 2H), 7.49-7.57 (m, 2H); ${ }^{13} \mathrm{C}$ NMR $\left(\mathrm{CDCl}_{3}\right) \delta$ 6.4, 56.1, 91.7, 92.0, 110.9, 112.6, 120.7, 124.3, 125.4, 127.7, 128.8, 130.1, 132.5, 133.8, 136.5, 160.2; IR (neat, $\mathrm{cm}^{-1}$ ) 3013, 2962, 2932, 2836, 2212, 1596, 1494; HRMS calcd for $\mathrm{C}_{16} \mathrm{H}_{14} \mathrm{OSe}$ 302.02099, found 302.02150.

1,4-Bis[(2-(methylselenyl)phenylethynyl]benzene (13). The product was obtained as a yellow solid: mp 159-161 ${ }^{\circ} \mathrm{C} ;{ }^{1} \mathrm{H}$ NMR $\left(\mathrm{CDCl}_{3}\right) \delta 2.37$ (s, 6H), 7.14-7.16 (m, 2H), 7.24-7.32 (m, 4H), 7.48 (d, $J=7.3 \mathrm{~Hz}, 2 \mathrm{H}), 7.56$ (s, 4H); ${ }^{13} \mathrm{C} \mathrm{NMR}\left(\mathrm{CDCl}_{3}\right) \delta$ 6.4, 89.9, 94.9, 123.3, 123.6, 
125.5, 127.6, 129.3, 131.7, 132.5, 136.8; IR (neat, $\mathrm{cm}^{-1}$ ) 3053, 2986, 2926, 2852, 2305, 1717, 1437, 1265; HRMS calcd for $\mathrm{C}_{24} \mathrm{H}_{18} \mathrm{Se}_{2} 465.97387$, found 465.97450 .

Methyl 3-(3-cyclohexylprop-1-ynyl)-4-(methylselenyl)benzoate (14). The product was obtained as a pale yellow liquid: ${ }^{1} \mathrm{H}$ NMR $\left(\mathrm{CDCl}_{3}\right) \delta$ 1.08-1.32 (m, 5H), 1.59-1.78 (m, 4H), 1.871.92 (m, 2H), 2.34-2.40 (m, 5H), 3.89 (s, 3H), 7.23 (d, $J=8.3 \mathrm{~Hz}, 1 \mathrm{H}), 7.83$ (dd, $J=8.3,1.9 \mathrm{~Hz}$, 1H), $7.97(\mathrm{~d}, J=1.7 \mathrm{~Hz}, 1 \mathrm{H}) ;{ }^{13} \mathrm{C} \mathrm{NMR}\left(\mathrm{CDCl}_{3}\right) \delta$ 6.1, 26.3, 26.4, 27.6, 32.9, 37.6, 52.3, 79.2, 97.0, 124.0, 125.9, 126.8, 128.8, 132.9, 143.7, 166.8; IR (neat, $\mathrm{cm}^{-1}$ ) 3058, 2923, 2850, 1710, 1460; HRMS calcd for $\mathrm{C}_{18} \mathrm{H}_{22} \mathrm{O}_{2}$ Se 350.07850, found 350.07902 .

General procedure for iodocyclization. To a solution of $0.25 \mathrm{mmol}$ of the alkyne and 3 $\mathrm{mL}$ of $\mathrm{CH}_{2} \mathrm{Cl}_{2}$, 1.1 equiv of $\mathrm{I}_{2}$ or ICl dissolved in $2 \mathrm{~mL}$ of $\mathrm{CH}_{2} \mathrm{Cl}_{2}$ was added gradually. The reaction mixture was allowed to stir at room temperature for the desired time. The excess $I_{2}$ or ICl was removed by washing with satd aq $\mathrm{Na}_{2} \mathrm{~S}_{2} \mathrm{O}_{3}$. The mixture was then extracted by diethyl ether $\left(3 \times 10 \mathrm{~mL}\right.$ ). The combined ether layers were dried over anhydrous $\mathrm{Na}_{2} \mathrm{SO}_{4}$ and concentrated under vacuum to yield the crude product, which was purified by flash chromatography on silica gel using ethyl acetate/hexanes as the eluent.

3-Iodo-2-octylbenzo[b] selenophene (15). The product was obtained as a yellow oil: ${ }^{1} \mathrm{H}$ NMR $\left(\mathrm{CDCl}_{3}\right) \delta 0.91(\mathrm{t}, J=6.9 \mathrm{~Hz}, 3 \mathrm{H}), 1.31-1.48(\mathrm{~m}, 10 \mathrm{H}), 1.71-1.79(\mathrm{~m}, 2 \mathrm{H}), 3.02(\mathrm{t}, J=7.8$ $\mathrm{Hz}, 2 \mathrm{H})$, 7.24-7.29 (m, 1H), 7.39-7.45 (m, 1H), 7.77-7.83 (m, 2H); ${ }^{13} \mathrm{C} \mathrm{NMR}\left(\mathrm{CDCl}_{3}\right) \delta$ 14.4, 22.9, 29.4, 29.5, 29.6, 31.6, 32.1, 36.1, 83.4, 125.3, 125.5, 125.7, 127.8, 139.1, 143.2, 148.2; IR 
(neat, $\mathrm{cm}^{-1}$ ) 3056, 2953, 2923, 2852, 1450, 1432, 1242; HRMS calcd for $\mathrm{C}_{16} \mathrm{H}_{21}$ ISe 419.98532, found 419.98620 .

3-Iodo-2-phenylbenzo[b]selenophene (16). The product was obtained as a yellow oil: ${ }^{1} \mathrm{H}$ $\operatorname{NMR}\left(\mathrm{CDCl}_{3}\right) \delta 7.34(\mathrm{t}, J=5.5 \mathrm{~Hz}, 1 \mathrm{H}), 7.41-7.51(\mathrm{~m}, 4 \mathrm{H}), 7.61-7.63(\mathrm{~m}, 2 \mathrm{H}), 7.83-7.85(\mathrm{~d}, J=$ 5.9 Hz, 1H), 7.93-7.95 (d, $J=6.0 \mathrm{~Hz}, 1 \mathrm{H}) ;{ }^{13} \mathrm{C} \mathrm{NMR}\left(\mathrm{CDCl}_{3}\right) \delta 82.9,125.3,125.9,126.0,128.6$, 128.9, 129.1, 130.2, 137.1, 140.7, 143.8, 144.7; IR (neat, $\mathrm{cm}^{-1}$ ) 3052, 2926, 2919, 1481, 1430 , 1239; HRMS calcd for $\mathrm{C}_{14} \mathrm{H}_{9} \mathrm{SeI} 383.89142$, found 383.89200 .

2-(Cyclohex-1-enyl)-3-iodobenzo[b]selenophene (17). The product was obtained as a yellow oil: ${ }^{1} \mathrm{H}$ NMR $\left(\mathrm{CDCl}_{3}\right) \delta$ 1.65-1.83 (m, 4H), 2.21-2.23 (m, 2H), 2.41-2.43 (m, 2H), 6.10$6.20(\mathrm{~m}, 1 \mathrm{H}), 7.25$ (t, $J=6.7 \mathrm{~Hz}, 1 \mathrm{H}), 7.41(\mathrm{t}, J=7.9 \mathrm{~Hz}, 1 \mathrm{H}), 7.75$ (d, $J=7.8 \mathrm{~Hz}, 1 \mathrm{H}), 7.83$ (d, $J=8.1 \mathrm{~Hz}, 1 \mathrm{H}) ;{ }^{13} \mathrm{C}$ NMR $\left(\mathrm{CDCl}_{3}\right) \delta 21.9,23.0,25.8,30.6,80.9,125.4,125.5,125.7,128.5$, 132.1, 134.7, 139.5, 143.5, 148.0; IR (neat, $\mathrm{cm}^{-1}$ ) 3018, 2917, 2848, 1461, 1215; HRMS calcd for $\mathrm{C}_{14} \mathrm{H}_{13} \mathrm{ISe} 387.92272$, found 387.92340.

4-(3-Iodobenzo[b]selenophen-2-yl)butanenitrile (18). The product was obtained as a yellow oil: ${ }^{1} \mathrm{H}$ NMR $\left(\mathrm{CDCl}_{3}\right) \delta 2.12(\mathrm{q}, J=7.3 \mathrm{~Hz}, 2 \mathrm{H}), 2.48(\mathrm{t}, J=7.2 \mathrm{~Hz}, 2 \mathrm{H}), 3.18(\mathrm{t}, J=7.5$ $\mathrm{Hz}, 2 \mathrm{H})$, 7.25-7.33 (m, 1H), 7.41-7.45 (m, 1H), 7.77-7.82 (m, 2H); ${ }^{13} \mathrm{C} \mathrm{NMR}\left(\mathrm{CDCl}_{3}\right) \delta$ 16.7, 27.0, 34.6, 85.1, 119.4, 125.6, 125.9, 126.0, 128.2, 139.3, 143.0, 144.0; IR (neat, $\mathrm{cm}^{-1}$ ) 3053, 2931, 2851, 2245, 1449, 1432; HRMS calcd for $\mathrm{C}_{12} \mathrm{H}_{10} \mathrm{INSe} 374.90232$, found 374.90290. 
Methyl 4-(3-iodobenzo[b]selenophen-2-yl)butanoate (19). The product was obtained as a yellow oil: ${ }^{1} \mathrm{H}$ NMR $\left(\mathrm{CDCl}_{3}\right) \delta 2.08$ (q, $\left.J=7.5 \mathrm{~Hz}, 2 \mathrm{H}\right), 2.45(\mathrm{t}, J=7.5 \mathrm{~Hz}, 2 \mathrm{H}), 3.07$ (t, $J=7.2$ Hz, 2H), 3.69 (s, 3H), 7.24-7.29 (m, 1H), 7.39-7.44 (m, 1H), 7.76-7.80 (m, $2 \mathrm{H})$; ${ }^{13} \mathrm{C}$ NMR $\left(\mathrm{CDCl}_{3}\right) \delta$ 26.5, 33.2, 35.2, 51.9, 84.2, 125.5, 125.6, 125.8, 129.3, 143.1, 146.4, 173.7; IR (neat, $\mathrm{cm}^{-1}$ ) 3058, 2918, 2843, 1730, 1431; HRMS calcd for $\mathrm{C}_{13} \mathrm{H}_{13} \mathrm{IO}_{2}$ Se 407.91255, found 407.91320.

2-(3-Iodobenzo[b]selenophen-2-yl)propan-2-ol (20). The product was obtained as a pale yellow solid: mp 91-93 ${ }^{\circ} \mathrm{C} ;{ }^{1} \mathrm{H}$ NMR $\left(\mathrm{CDCl}_{3}\right) \delta 1.77$ (s, 6H), 2.69 (s, 1H), 7.16-7.22 (m, 1H), 7.31-7.37 (m, 1H), 7.70-7.78 (m, 2H); ${ }^{13} \mathrm{C}$ NMR $\left(\mathrm{CDCl}_{3}\right) \delta$ 29.8, 75.0, 76.0, 125.2, 125.4, 125.6, 128.1, 138.4, 145.1, 157.5; IR (neat, $\mathrm{cm}^{-1}$ ) 3398, 2973, 2922, 2850, 1447, 1433, 1239; HRMS calcd for $\mathrm{C}_{11} \mathrm{H}_{11} \mathrm{IOSe} 365.90197$, found 365.90242.

2-(Triethylsilyl)benzo[b]selenophene (21). The product was obtained as a colorless oil: ${ }^{1} \mathrm{H}$ NMR $\left(\mathrm{CDCl}_{3}\right) \delta$ 0.99-1.08 (m, 15H), 7.24-7.34 (m, 1H), 7.41-7.47 (m, 1H), 7.87-7.93 (m, 2H); ${ }^{13} \mathrm{C} \mathrm{NMR}\left(\mathrm{CDCl}_{3}\right) \delta 4.2,7.7,90.2,125.0,125.4,125.5,128.2,142.3,142.4,145.1$; IR (neat, $\mathrm{cm}^{-1}$ ) 3053, 2952, 2872, 1473, 1416, 1230; HRMS calcd for $\mathrm{C}_{14} \mathrm{H}_{19}$ ISiSe 421.94660, found 421.94720 .

2-Cyclohexylmethyl-3-iodobenzo[b]selenophene (22). The product was obtained as a yellow oil: ${ }^{1} \mathrm{H}$ NMR $\left(\mathrm{CDCl}_{3}\right) \delta$ 1.07-1.25 (m, 6H), 1.67-1.84 (m, 5H), $2.92(\mathrm{~d}, J=7.0 \mathrm{~Hz}, 2 \mathrm{H})$, $7.24(\mathrm{t}, J=7.1 \mathrm{~Hz}, 1 \mathrm{H}), 7.39(\mathrm{t}, J=7.3 \mathrm{~Hz}, 1 \mathrm{H}), 7.75-7.81(\mathrm{~m}, 2 \mathrm{H}) ;{ }^{13} \mathrm{C} \mathrm{NMR}\left(\mathrm{CDCl}_{3}\right) \delta 26.4$, 26.6, 33.3, 40.8, 43.5, 84.5, 125.4, 125.5, 125.6, 127.9, 139.4, 143.2, 146.8; IR (neat, $\mathrm{cm}^{-1}$ ) 2920, 2849, 1446, 1432, 1242; HRMS calcd for $\mathrm{C}_{15} \mathrm{H}_{17} \mathrm{ISe} 403.95402$, found 403.95458. 
Methyl 2-cyclohexylmethyl-3-iodobenzo[b]selenophene-5-carboxylate (23). The product was obtained as a white solid: mp 150-152 ${ }^{\circ} \mathrm{C} ;{ }^{1} \mathrm{H}$ NMR $\left(\mathrm{CDCl}_{3}\right) \delta 1.08-1.26$ (m, 5H), 1.61-1.84 (m, 6H), 2.95 (d, $J=7.0 \mathrm{~Hz}, 2 \mathrm{H}), 3.98$ (s, 3H), 7.82-7.93 (m, 2H), 8.47 (s, $1 \mathrm{H}) ;{ }^{13} \mathrm{C}$ NMR $\left(\mathrm{CDCl}_{3}\right) \delta$ 26.3, 26.5, 33.3, 40.7, 43.5, 52.5, 84.6, 125.5, 125.7, 127.9, 129.3, 143.3, 144.8, 148.3, 167.3; IR (neat, $\mathrm{cm}^{-1}$ ) 2976, 2928, 2859, 1726, 1444, 1382; HRMS calcd for $\mathrm{C}_{17} \mathrm{H}_{19} \mathrm{IO}_{2} \mathrm{Se}$ 461.95948, found 461.96021.

3-Iodo-2-(4-nitrophenyl)benzo[b]selenophene (24). The product was obtained as an orange solid: mp 147-149 ${ }^{\circ} \mathrm{C} ;{ }^{1} \mathrm{H}$ NMR $\left(\mathrm{CDCl}_{3}\right) \delta$ 7.36-7.41 (m, 1H), 7.49-7.54 (m, 1H), 7.78 (d, $J=8.8 \mathrm{~Hz}, 2 \mathrm{H}), 7.87(\mathrm{~d}, J=7.8 \mathrm{~Hz}, 1 \mathrm{H}), 7.95(\mathrm{~d}, J=7.7 \mathrm{~Hz}, 1 \mathrm{H}), 8.30(\mathrm{~d}, J=8.7 \mathrm{~Hz}, 2 \mathrm{H}) ;{ }^{13} \mathrm{C}$ NMR $\left(\mathrm{CDCl}_{3}\right) \delta 84.8,123.9,125.5,126.5,126.7,129.6,131.3,140.9,141.5,143.6,143.7$, 147.8; IR (neat, $\mathrm{cm}^{-1}$ ) 3004, 2977, 2872, 1445, 1383; HRMS calcd for $\mathrm{C}_{14} \mathrm{H}_{8} \mathrm{INO}_{2} \mathrm{Se} 428.87648$, found 428.87710 .

3-Iodo-2-(2-methoxyphenyl)benzo[b]selenophene (25). The product was obtained as a yellow solid: mp 94-96 ${ }^{\circ} \mathrm{C} ;{ }^{1} \mathrm{H}$ NMR $\left(\mathrm{CDCl}_{3}\right) \delta 3.83$ (s, 3H), 6.99-7.07 (m, 2H), 7.28-7.47 (m, 4H), 7.83 (d, $J=7.9 \mathrm{~Hz}, 1 \mathrm{H}), 7.89$ (d, $J=8.1 \mathrm{~Hz}, 1 \mathrm{H}) ;{ }^{13} \mathrm{C} \mathrm{NMR}\left(\mathrm{CDCl}_{3}\right) \delta 55.9,86.0,111.6$, 120.6, 125.4, 125.7, 126.1, 128.8, 130.8, 132.4, 141.2, 141.7, 143.2, 156.9; IR (neat, $\mathrm{cm}^{-1}$ ) 3056, 3004, 2934, 2843, 1597, 1482, 1432, 1249; HRMS calcd for $\mathrm{C}_{15} \mathrm{H}_{11} \mathrm{IOSe}$ 413.90199, found 413.90260 . 
1,4-Bis(3-iodobenzo[b]selenophen-2-yl)benzene (26). The product was purified by washing the crude product with hexanes and recrystallizing it from benzene. The product was obtained as a yellow solid: $\mathrm{mp}>260{ }^{\circ} \mathrm{C}$; IR $\left(\mathrm{KBr}, \mathrm{cm}^{-1}\right)$ 3067, 3044, 1537, 1478, 1428, 1237; HRMS calcd for $\mathrm{C}_{22} \mathrm{H}_{12} \mathrm{Se}_{2} \mathrm{I}_{2}$ 689.73586, found 689.73730; Anal. Calcd for $\mathrm{C}_{22} \mathrm{H}_{12} \mathrm{Se}_{2} \mathrm{I}_{2}$ : C, 38.31; H, 1.73. Found: C, 38.75; H, 1.72.

General procedure for bromocyclization. To a solution of $0.25 \mathrm{mmol}$ of the alkyne and $3 \mathrm{~mL}$ of $\mathrm{CH}_{2} \mathrm{Cl}_{2}, 1.1$ equiv of $\mathrm{Br}_{2}$ or 2.2 equiv of $\mathrm{NBS}$ dissolved in $2 \mathrm{~mL}$ of $\mathrm{CH}_{2} \mathrm{Cl}_{2}$ was added gradually. The reaction mixture was allowed to stir at room temperature for the desired time. The excess $\mathrm{Br}_{2}$ or NBS was removed by washing with satd aq $\mathrm{Na}_{2} \mathrm{~S}_{2} \mathrm{O}_{3}$. The mixture was then extracted by diethyl ether ( $3 \times 10 \mathrm{~mL}$ ). The combined ether layers were dried over anhydrous $\mathrm{Na}_{2} \mathrm{SO}_{4}$ and concentrated under vacuum to yield the crude product, which was purified by flash chromatography on silica gel using ethyl acetate/hexanes as the eluent.

3-Bromo-2-phenylbenzo[b]selenophene (28). The product was obtained as a yellow oil: ${ }^{1} \mathrm{H}$ NMR $\left(\mathrm{CDCl}_{3}\right) \delta$ 7.34-7.53 (m, 5H), 7.68-7.72 (m, 2H), 7.87 (d, $\left.J=7.9 \mathrm{~Hz}, 1 \mathrm{H}\right), 7.95$ (d, $J=7.5 \mathrm{~Hz}, 1 \mathrm{H}) ;{ }^{13} \mathrm{C} \mathrm{NMR}\left(\mathrm{CDCl}_{3}\right) \delta 106.9,125.4,125.8,125.9,126.3,128.8,128.9,130.1$, 135.2, 139.2, 140.9, 141.2; IR (neat, $\mathrm{cm}^{-1}$ ) 3059, 3016, 2925, 1484, 1216; HRMS calcd for $\mathrm{C}_{14} \mathrm{H}_{9} \mathrm{BrSe} 335.90528$, found 335.90562.

3-Bromo-2-octylbenzo[b]selenophene (31). The product was obtained as a yellow oil: ${ }^{1} \mathrm{H}$ $\operatorname{NMR}\left(\mathrm{CDCl}_{3}\right) \delta 0.81(\mathrm{t}, J=6.3 \mathrm{~Hz}, 3 \mathrm{H}), 1.10-1.40(\mathrm{~m}, 10 \mathrm{H}), 1.65$ (quintet, $J=7.7 \mathrm{~Hz}, 2 \mathrm{H}$ ), 2.91 (t, $J=7.7 \mathrm{~Hz}, 2 \mathrm{H}), 7.18-7.22(\mathrm{~m}, 1 \mathrm{H}), 7.31-7.37(\mathrm{~m}, 1 \mathrm{H}), 7.72(\mathrm{~d}, J=8.0 \mathrm{~Hz}, 2 \mathrm{H}) ;{ }^{13} \mathrm{C}$ NMR $\left(\mathrm{CDCl}_{3}\right) \delta 14.4,22.9,29.3,29.4,29.6,31.4,32.1,32.5,107.6,125.1,125.2,125.4,125.6$, 
138.0, 140.5, 144.5; IR (neat, $\mathrm{cm}^{-1}$ ) 3059, 2926, 2854, 1452, 1434, 1214; HRMS calcd for $\mathrm{C}_{16} \mathrm{H}_{21} \mathrm{BrSe}$ 371.99918, found 371.99990.

3-Bromo-2(cyclohex-1-enyl)benzo[b]selenophene (33). The product was obtained as a yellow oil: ${ }^{1} \mathrm{H}$ NMR $\left(\mathrm{CDCl}_{3}\right) \delta$ 1.60-1.85 (m, 4H), 2.20-2.30 (m, 2H), 2.45-2.55 (m, 2H), 6.22$6.28(\mathrm{~m}, 1 \mathrm{H}), 7.30(\mathrm{t}, J=7.3 \mathrm{~Hz}, 1 \mathrm{H}), 7.45(\mathrm{t}, J=7.2 \mathrm{~Hz}, 1 \mathrm{H}) ;{ }^{13} \mathrm{C} \mathrm{NMR}\left(\mathrm{CDCl}_{3}\right) \delta$ 21.9, 23.1, 25.9, 30.3, 105.0, 125.3, 125.4, 125.5, 125.7, 132.1, 132.8, 137.9, 141.1, 144.2; IR (neat, $\mathrm{cm}^{-1}$ ) 3018, 2932, 1450, 1215; HRMS calcd for $\mathrm{C}_{14} \mathrm{H}_{13} \mathrm{BrSe} 339.93658$, found 339.93720 .

General procedure for the $\mathbf{P h S e C l}$ and $\mathbf{P h S e B r}$ cyclizations. To a solution of 0.25 mmol of the alkyne and $\mathrm{CH}_{2} \mathrm{Cl}_{2}(3 \mathrm{~mL}), 0.375 \mathrm{mmol}$ of $\mathrm{PhSeBr}$ or $\mathrm{PhSeCl}$ dissolved in $2 \mathrm{~mL}$ of $\mathrm{CH}_{2} \mathrm{Cl}_{2}$ was added dropwise. The mixture was allowed to stir at room temperature for the desired time. The reaction mixture was washed with $20 \mathrm{~mL}$ of water and extracted with diethyl ether ( $3 \times 10 \mathrm{~mL}$ ). The combined ether layers were dried over anhydrous $\mathrm{Na}_{2} \mathrm{SO}_{4}$ and concentrated under vacuum to yield the crude product, which was further purified by flash chromatography on silica gel using ethyl acetate/hexanes as the eluent.

2-Phenyl-3-(phenylselenyl)benzo[b]selenophene (27). The product was obtained as a yellow oil: ${ }^{1} \mathrm{H}$ NMR $\left(\mathrm{CDCl}_{3}\right) \delta$ 7.07-7.17 (m, 5H), 7.29-7.41 (m, 5H), 7.55-7.58 (m, 2H), 7.897.97 (m, 2H); ${ }^{13} \mathrm{C}$ NMR $\left(\mathrm{CDCl}_{3}\right) \delta$ 118.6, 125.3, 125.5, 125.6, 126.1, 127.8, 128.4, 128.9, 129.2, 129.4, 130.3, 133.2, 136.4, 141.2, 144.1, 153.2 ; IR (neat, $\mathrm{cm}^{-1}$ ) 3056, 2917, 2848, 1476, 1431, 1215; HRMS calcd for $\mathrm{C}_{20} \mathrm{H}_{14} \mathrm{Se}_{2} 411.94547$, found 411.94630 . 
2-Octyl-3-(phenylselenyl)benzo[b]selenophene (30). The product was obtained as a yellow oil: ${ }^{1} \mathrm{H}$ NMR $\left(\mathrm{CDCl}_{3}\right) \delta 0.86(\mathrm{t}, J=6.5 \mathrm{~Hz}, 3 \mathrm{H}), 1.23-1.37(\mathrm{~m}, 10 \mathrm{H}), 1.69$ (quintet, $J=$ $7.3 \mathrm{~Hz}, 2 \mathrm{H}), 3.21(\mathrm{t}, J=7.4 \mathrm{~Hz}, 2 \mathrm{H}), 7.08-7.14(\mathrm{~m}, 5 \mathrm{H}), 7.21-7.34(\mathrm{~m}, 2 \mathrm{H}), 7.83-7.89(\mathrm{~m}, 2 \mathrm{H})$; ${ }^{13} \mathrm{C}$ NMR $\left(\mathrm{CDCl}_{3}\right) \delta 14.4,22.9,29.4,29.6,32.1,32.7,33.7,118.8,124.9,125.3,125.5,126.0$, 126.6, 129.0, 129.4,132.9, 139.9, 143.7, 157.8; IR (neat, $\mathrm{cm}^{-1}$ ) 3058, 2925, 2853, 1577, 1476, 1215; HRMS calcd for $\mathrm{C}_{22} \mathrm{H}_{26} \mathrm{Se}_{2}$ 448.03937, found 448.04010.

2-(Cyclohex-1-enyl)-3-(phenylselenyl)benzo[b]selenophene (32). The product was obtained as a yellow solid: ${ }^{1} \mathrm{H}$ NMR $\left(\mathrm{CDCl}_{3}\right) \delta$ 1.52-1.77 (m, 4H), 2.14-2.20 (m, 2H), 2.43-2.45 (m, 2H), 6.00-6.03 (m, 1H), 7.08-7.18 (m, 5H), 7.21-7.32 (m, 2H), 7.81-7.85 (m, 2H); ${ }^{13} \mathrm{C}$ NMR $\left(\mathrm{CDCl}_{3}\right) \delta$ 21.9, 23.1, 25.9, 31.1, 116.7, 125.1, 125.3, 125.9, 127.1, 129.2, 129.3, 131.9, 133.5, 134.0, 140.0, 143.9, 156.3; IR (neat, $\mathrm{cm}^{-1}$ ) 3017, 2932, 2859, 1476, 1215; HRMS calcd for $\mathrm{C}_{20} \mathrm{H}_{18} \mathrm{Se}_{2}$ 417.98082, found 417.98160.

(2-Phenylbenzo[b]selenophen-3-yl)mercury(II) chloride (29). To a solution of 0.25 mmol of 10 in glacial acetic acid $(5 \mathrm{~mL})$ was added $0.27 \mathrm{mmol}$ of $\mathrm{Hg}(\mathrm{OAc})_{2}$ at $0{ }^{\circ} \mathrm{C}$. The resulting solution was stirred at $0{ }^{\circ} \mathrm{C}$ for $1 \mathrm{~h}$ and room temperature for an additional $1 \mathrm{~h}$. The resulting mixture was poured into an ice cold satd $\mathrm{NaCl}$ solution $(10 \mathrm{~mL})$ with vigorous stirring. The resulting mixture was filtered and the residue was washed with water $(5 \mathrm{~mL})$ and hexane $(10$ $\mathrm{mL}$ ). The residue was dried and recrystallized using $\mathrm{CHCl}_{3}$ to give the pure product as a white solid: mp 207-209 ${ }^{\circ} \mathrm{C}:{ }^{1} \mathrm{H}$ NMR $\left(\mathrm{CDCl}_{3}\right) \delta$ 7.28 -7.43 (m, 2H), 7.39-7.52 (m, 4H), 7.63-7.66 (m, 2H), 7.87 (d, $J=7.3 \mathrm{~Hz}, 1 \mathrm{H}), 7.95$ (d, $J=7.8 \mathrm{~Hz}, 1 \mathrm{H})$; IR $\left(\mathrm{KBr}, \mathrm{cm}^{-1}\right.$ ) 3063, 3015, 2989, 1583, 1477, 1441; Anal. Calcd for $\mathrm{C}_{14} \mathrm{H}_{9} \mathrm{SeHgCl}$ C, 34.16; H, 1.84. Found: C, 34.01; H, 1.71. 
2-Octyl-3-phenylbenzo[b]selenophene (36). To the stirred solution of 5:1 DMF/water (5 $\mathrm{mL}), 0.25 \mathrm{mmol}$ of 3-iodo-2-octylbenzo[b]selenophene (15), $\mathrm{Pd}\left(\mathrm{PPh}_{3}\right)_{4}(5 \mathrm{~mol} \%)$ and 0.50 mmol of $\mathrm{K}_{2} \mathrm{CO}_{3}, 0.37 \mathrm{mmol}$ of phenylboronic acid was added. The reaction flask was sealed and flushed with Ar. After overnight stirring at $100{ }^{\circ} \mathrm{C}$, the resulting mixture was filtered, washed with satd aq $\mathrm{NH}_{4} \mathrm{Cl}$ and extracted with diethyl ether $(3 \mathrm{x} 10 \mathrm{~mL}$ ). The combined ether layers were dried over anhydrous $\mathrm{Na}_{2} \mathrm{SO}_{4}$ and concentrated under vacuum. The crude product was purified by flash chromatography on silica gel using hexanes as the eluent to furnish the desired product as colorless oil: ${ }^{1} \mathrm{H}$ NMR $\left(\mathrm{CDCl}_{3}\right) \delta 0.87(\mathrm{t}, J=7.1 \mathrm{~Hz}, 3 \mathrm{H}), 1.20-1.34(\mathrm{~m}, 10 \mathrm{H}), 1.67$ (quintet, $J=7.6 \mathrm{~Hz}, 2 \mathrm{H}), 2.83(\mathrm{t}, J=7.8 \mathrm{~Hz}, 2 \mathrm{H}), ;{ }^{13} \mathrm{C} \mathrm{NMR}\left(\mathrm{CDCl}_{3}\right) \delta 14.3,22.9,29.3,29.4$, 29.5, 31.1, 32.1, 33.0, 124.2, 124.6, 124.9, 125.5, 127.5, 128.7, 130.4, 136.9, 137.1, 139.6, 143.4, 147.6 ; IR (neat, $\mathrm{cm}^{-1}$ ) 3055, 2925, 2853, 1453, 1434, 1215; HRMS calcd for $\mathrm{C}_{22} \mathrm{H}_{36} \mathrm{Se}$ 370.11997, found 370.12059.

2-Phenyl-3-(phenylethynyl)benzo[b]selenophene (37). To a solution of $\mathrm{Et}_{3} \mathrm{~N}$ (5 mL), 0.25 mmol of 3-iodo-2-phenylbenzo[b]selenophene (16) and $\mathrm{PdCl}_{2}\left(\mathrm{PPh}_{3}\right)_{2}(2 \mathrm{~mol} \%)$ (stirring for 5 min beforehand), $\mathrm{CuI}$ (1 mol \%) was added and the flask was sealed and flushed with Ar. 0.38 Mmol of phenylacetylene dissolved in $2 \mathrm{~mL}$ of $\mathrm{Et}_{3} \mathrm{~N}$ was then added dropwise and the reaction mixture was allowed to stir at room temperature for $24 \mathrm{~h}$. After the reaction was over, the resulting solution was filtered, washed with satd aq $\mathrm{NaCl}$ and extracted with diethyl ether (3 x 10 $\mathrm{mL}$ ). The combined ether fractions were dried over anhydrous $\mathrm{Na}_{2} \mathrm{SO}_{4}$ and concentrated under vacuum to yield the crude product. The crude product was purified by flash chromatography on silica gel using hexanes as the eluent. The product was obtained as a colorless oil: ${ }^{1} \mathrm{H}$ NMR 
$\left(\mathrm{CDCl}_{3}\right) \delta$ 7.31-7.43 (m, 5H), 7.45-7.52 (m, 3H), 7.55-7.59 (m, 2H), $7.86(\mathrm{~d}, J=5.9 \mathrm{~Hz}, 1 \mathrm{H})$, $7.99(\mathrm{~d}, J=5.7 \mathrm{~Hz}, 1 \mathrm{H}), 8.08(\mathrm{~d}, J=5.8 \mathrm{~Hz}, 1 \mathrm{H}) ;{ }^{13} \mathrm{C} \mathrm{NMR}\left(\mathrm{CDCl}_{3}\right) \delta$ 85.6, 93.8, 116.9, 123.6, 125.3, 125.4, 125.6, 125.7, 128.6, 128.7, 128.9, 129, 129.2, 131.8, 136.0, 139.1, 143.3, 151.1; IR (neat, $\mathrm{cm}^{-1}$ ) 3053, 2986, 2304, 1421, 1265; HRMS calcd for $\mathrm{C}_{22} \mathrm{H}_{14} \mathrm{Se}$ 358.02062, found 358.02672. 


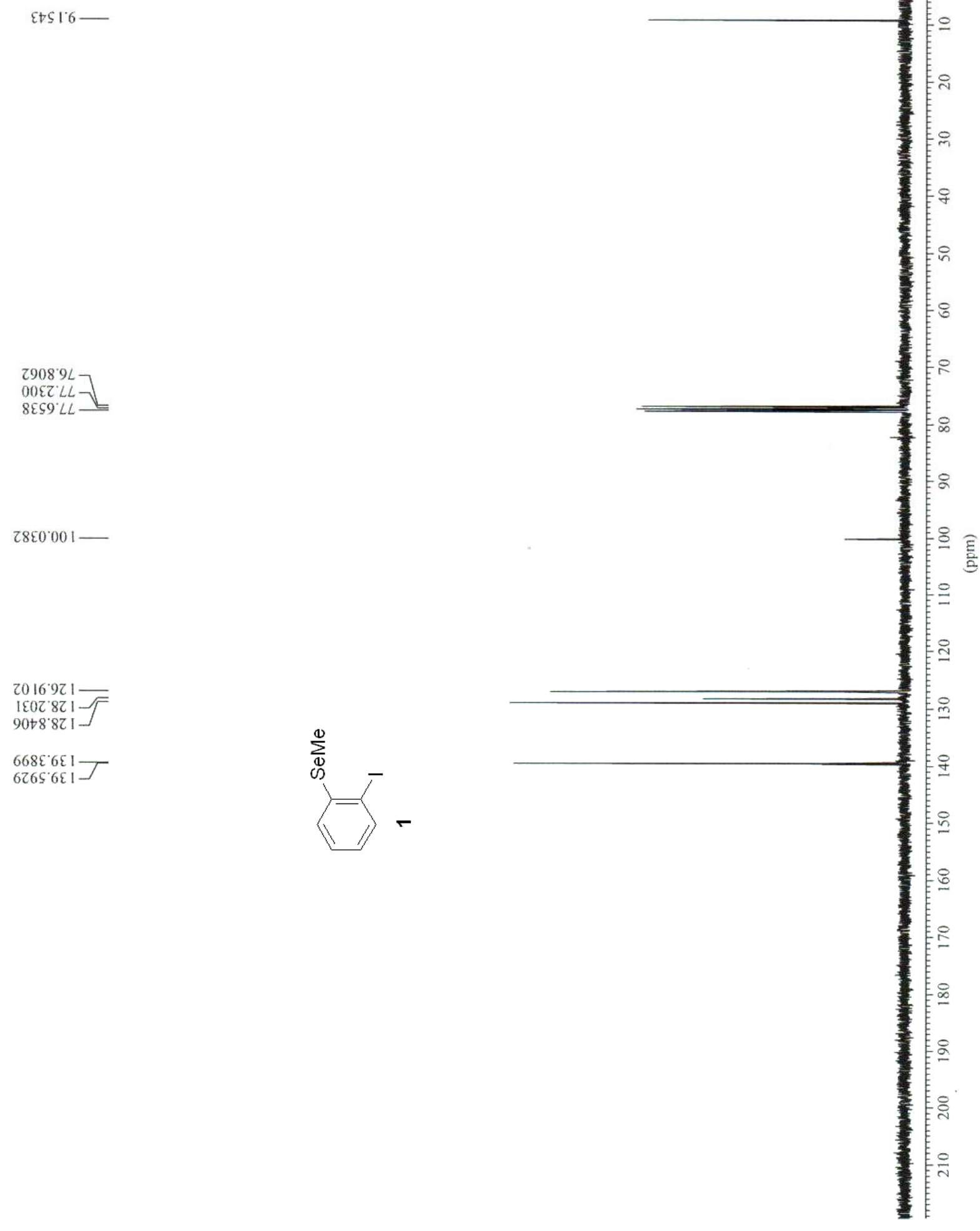




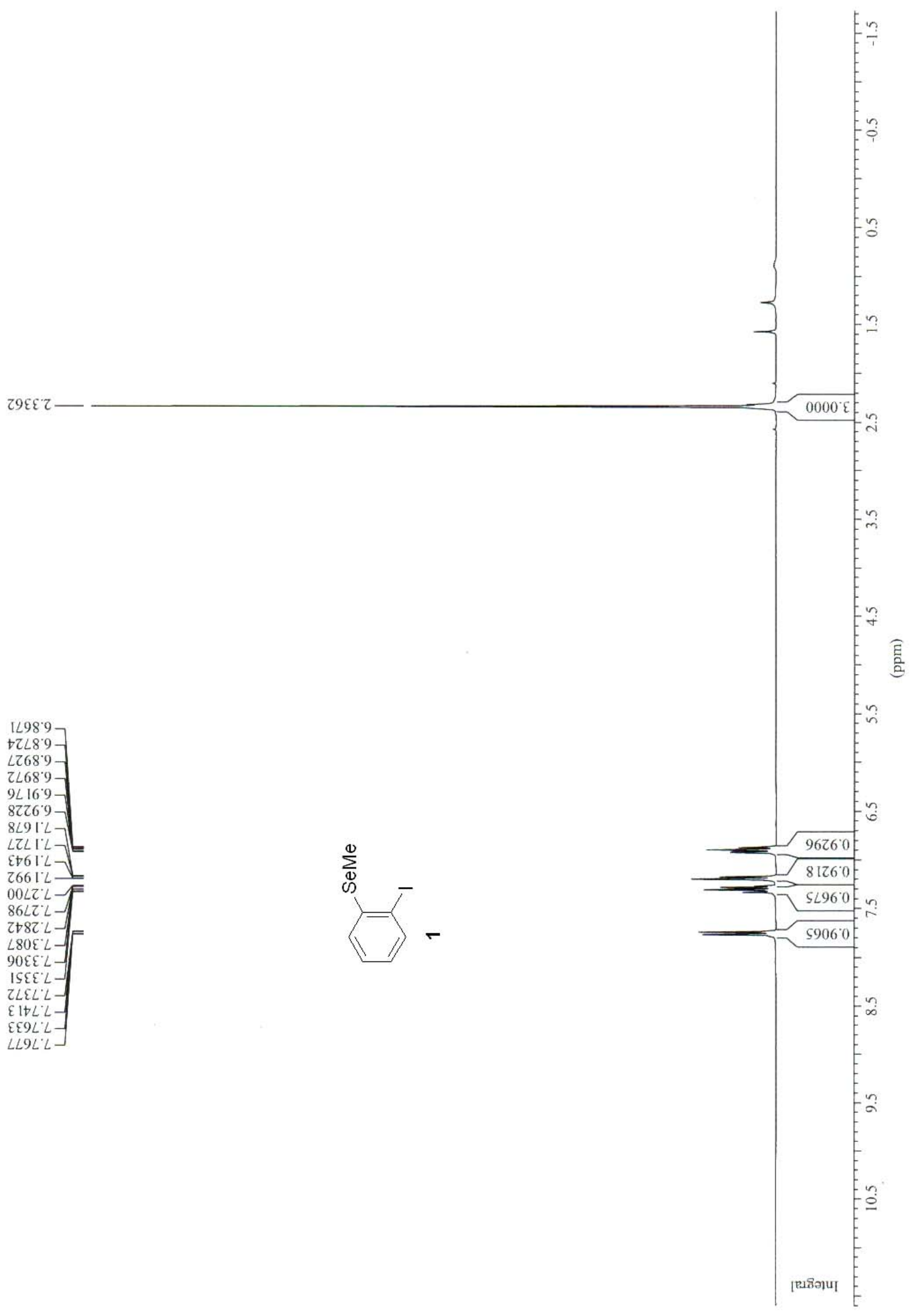


ธ $96 £ 6$

L8ttizs

$2908^{\circ} 9 L$

$00 \varepsilon \tau, L L$
$8 \varepsilon \subseteq 9 . L L$

$+28286$

I $\varepsilon 06.921-$
$0 \$ 82.821-F$
L9\$5 $621-$

$6866^{\circ} 6 \varepsilon 1-$

$06 \angle I L+1-$

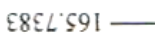
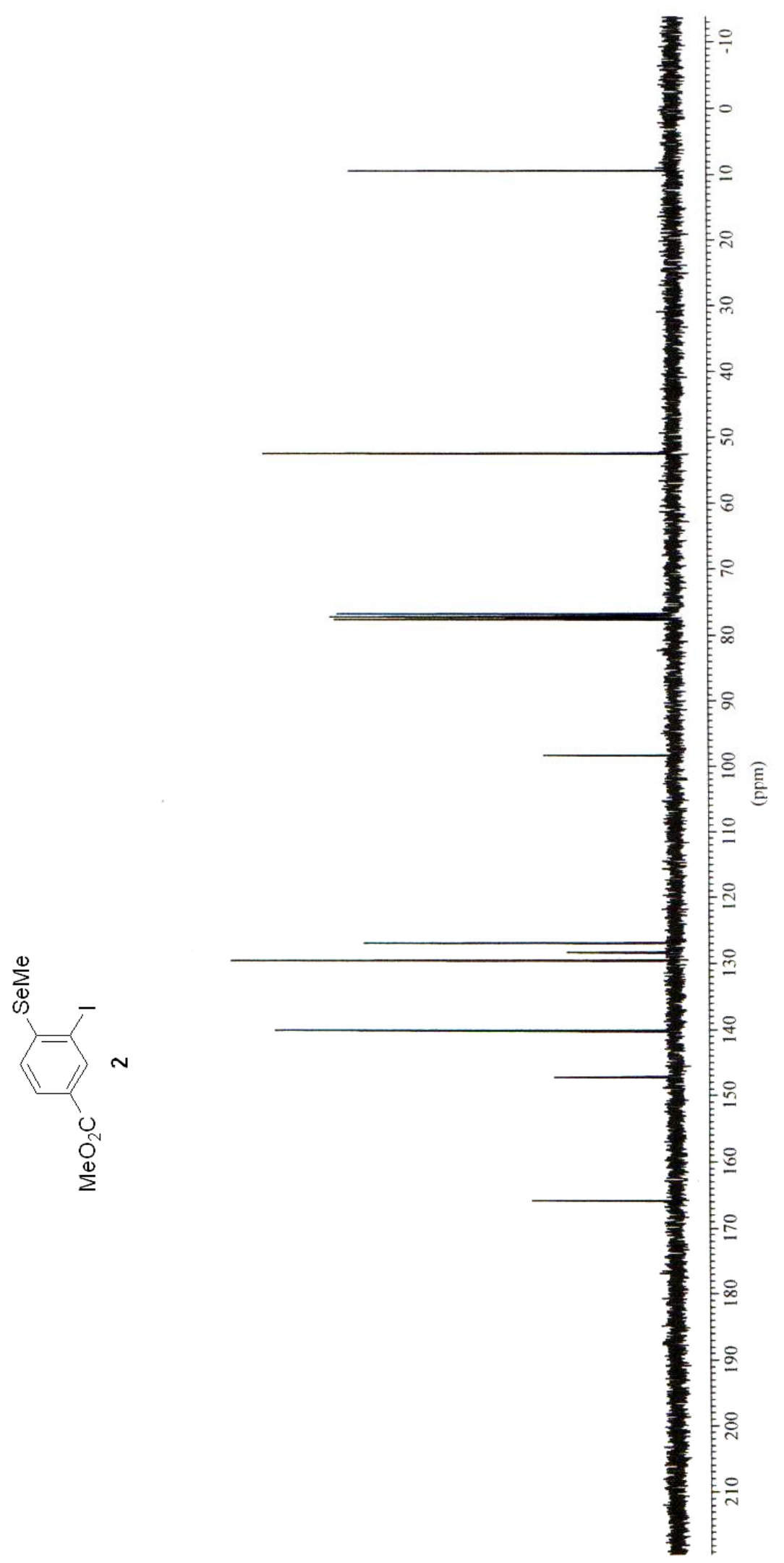


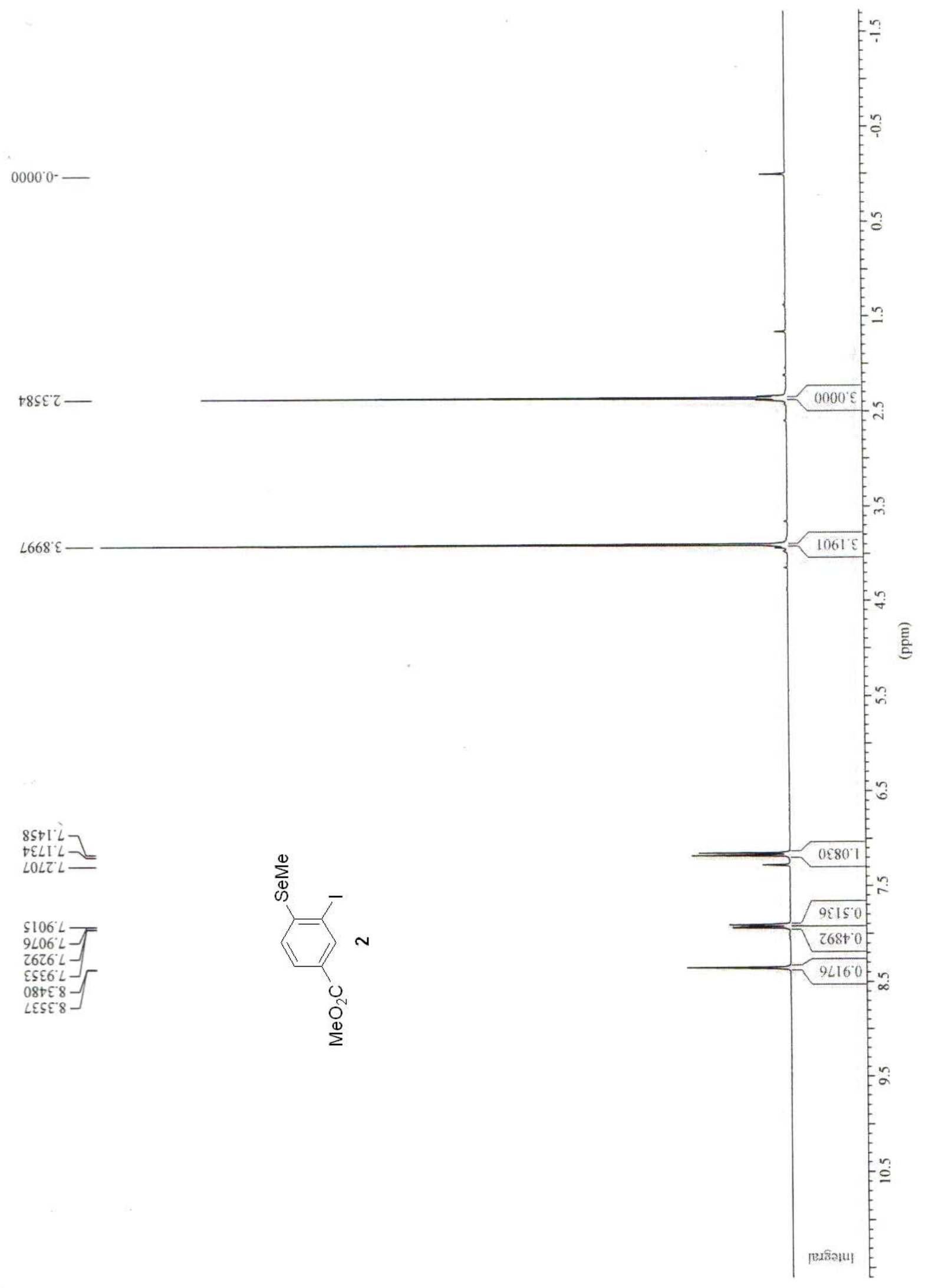




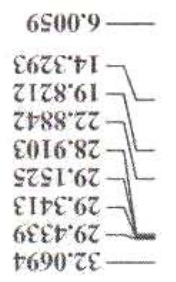

$2908^{*} 9 L$

$008 Z^{\prime} L L-f$

$8599^{\circ} \mathrm{LL}-$

$2 S 87^{\circ} 6 L-$

L288'96-

$260 t 50 \mathrm{CI}$

I0tISZI $-\sqrt{\Gamma}$

$\left.\$ 880^{\circ} 8 \mathrm{CI}\right]$

090天zรI
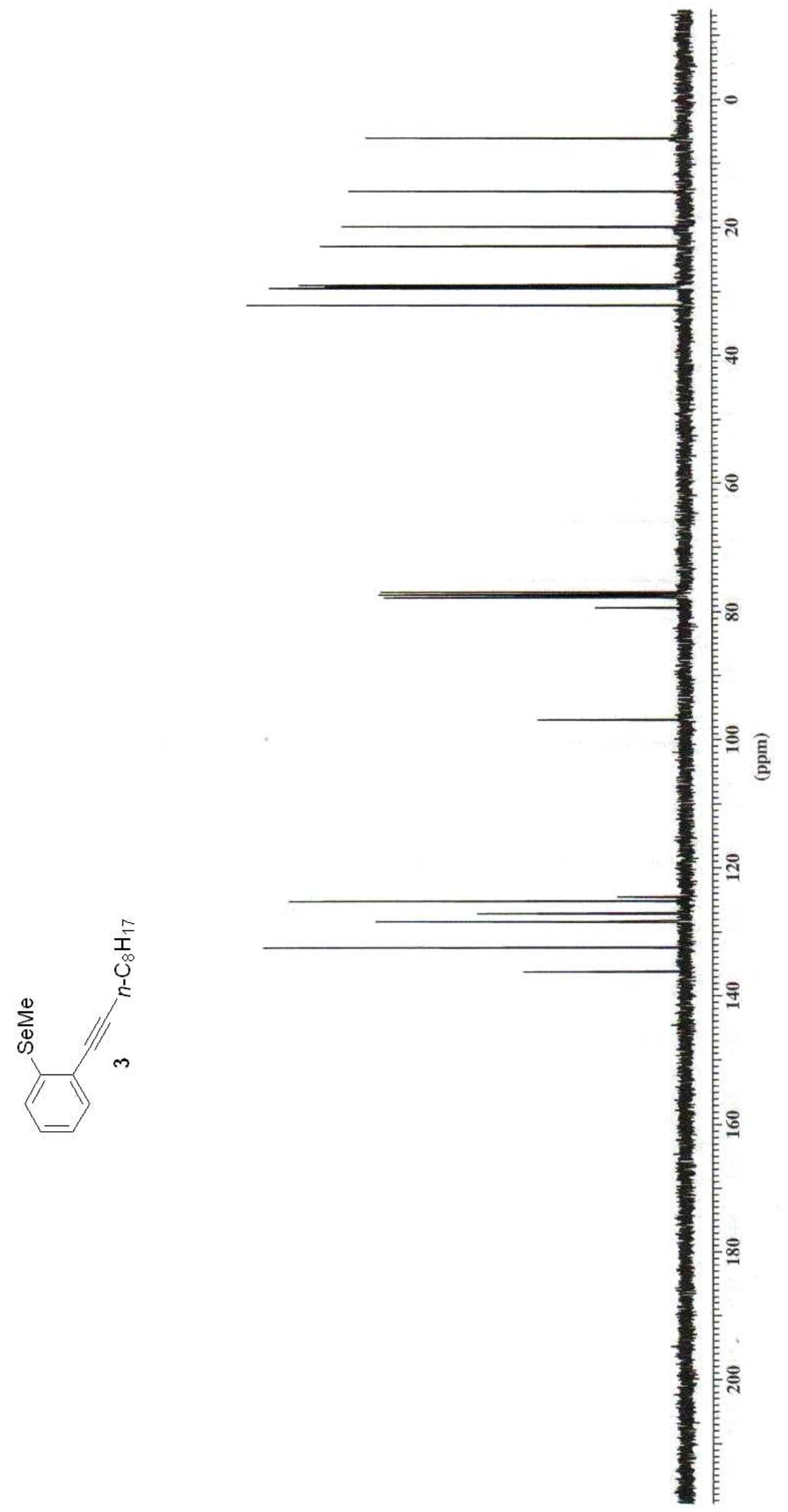


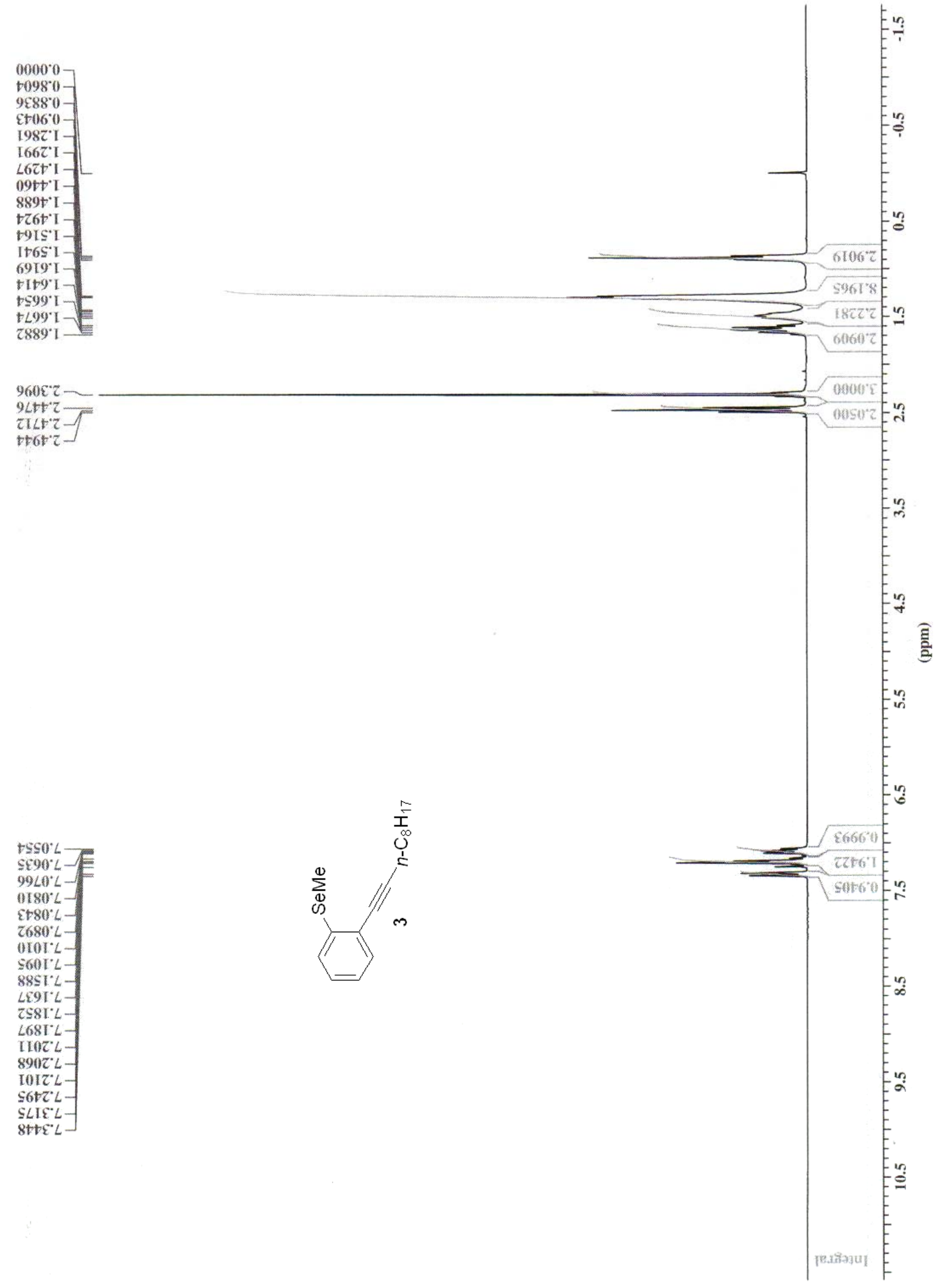



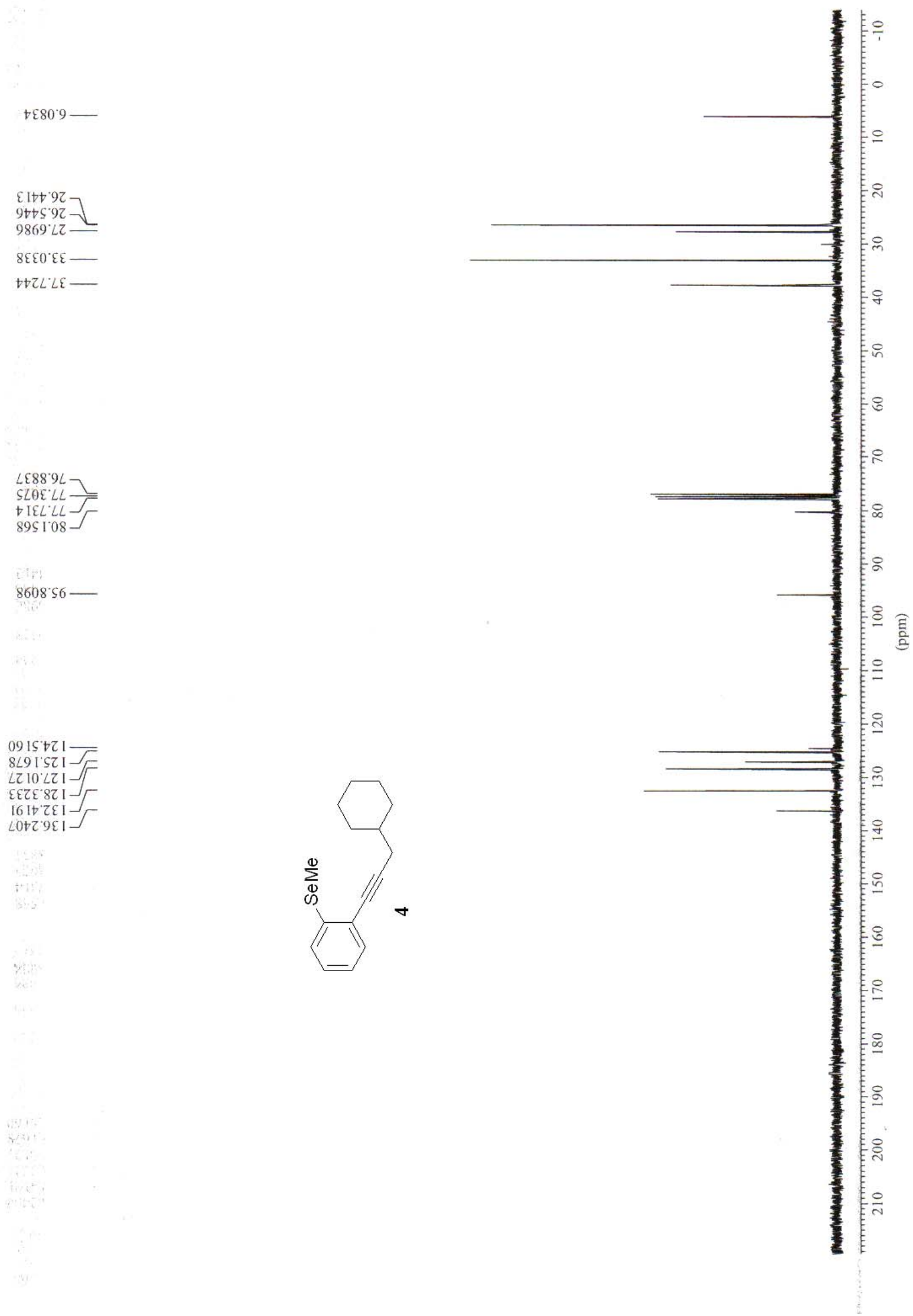

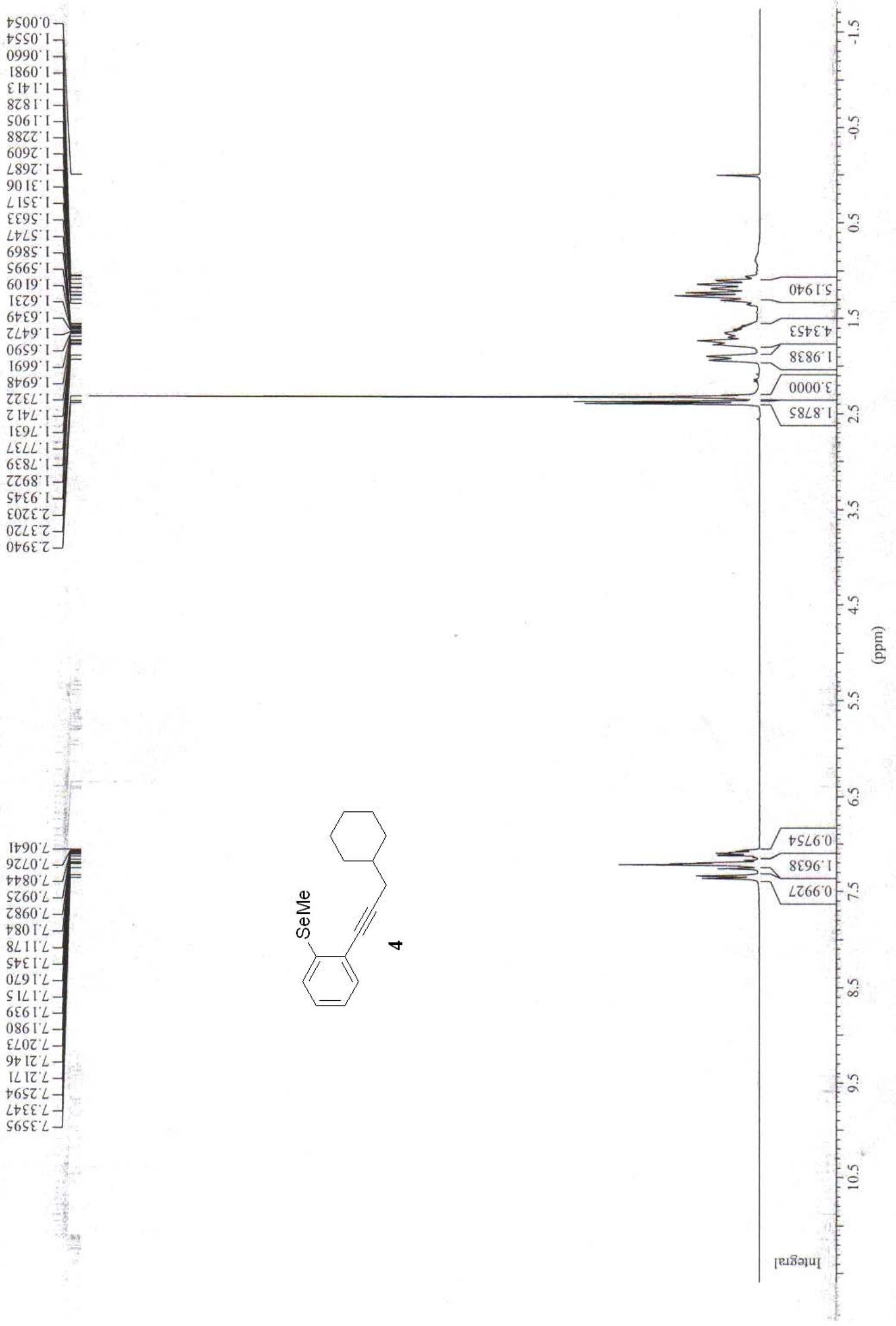


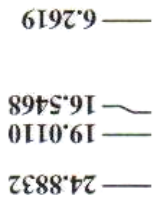

$2986^{\circ} 9 L$

$\angle t 0 \varepsilon \cdot L L-$

$\varepsilon 619^{\circ} \angle L \perp$

$6 \mathrm{ttI}+\mathrm{L} 8$

$0 \varepsilon z z^{*} \varepsilon 6$

zISS" $6 \mathrm{II}$

8I\& "EZI -

$\angle 8 Z \varepsilon^{\circ} \subseteq Z I-F$

L8I6.82I I

t90t'z\&I

9LEะ $9 \varepsilon \mathrm{I}$

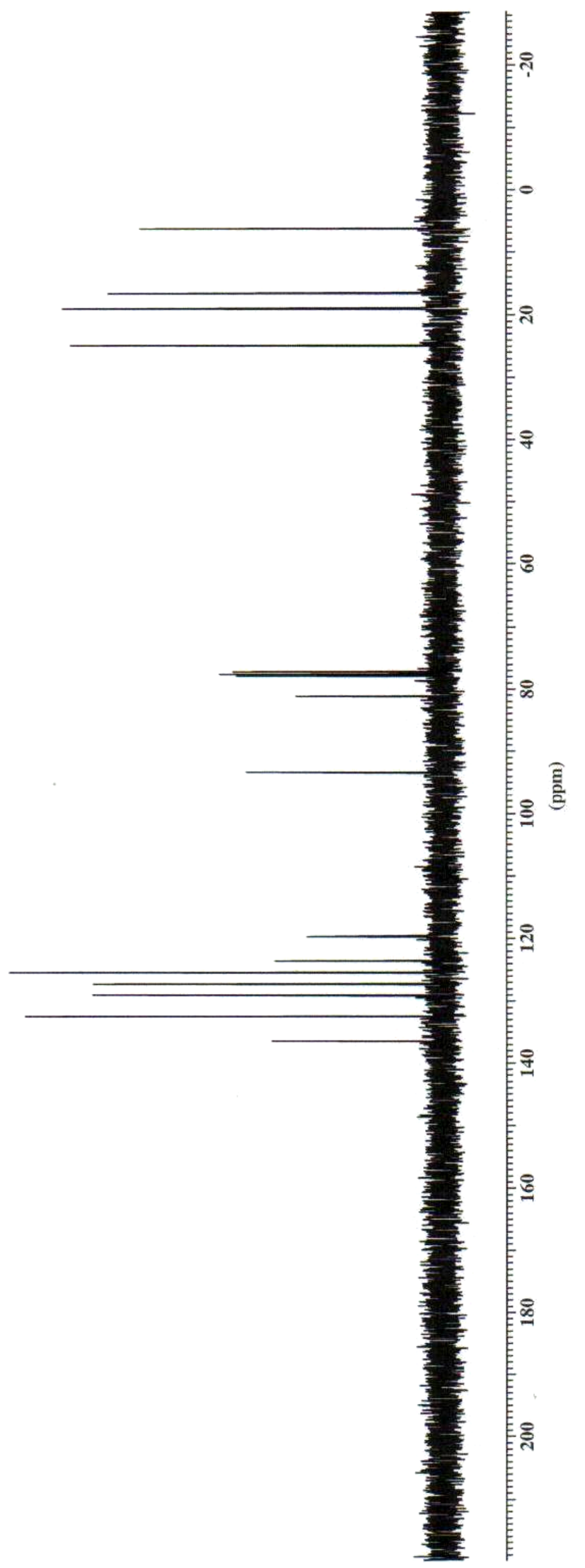




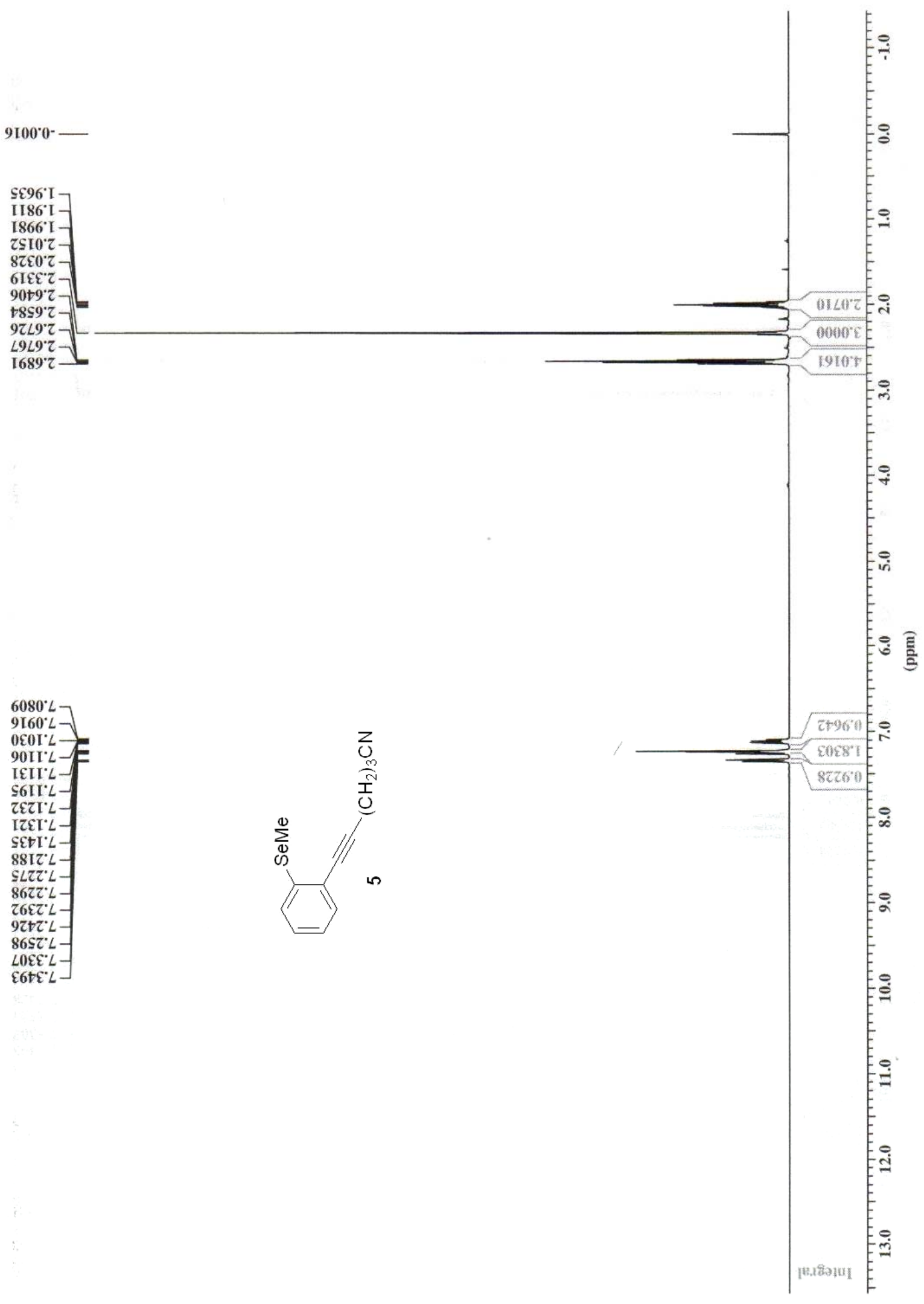



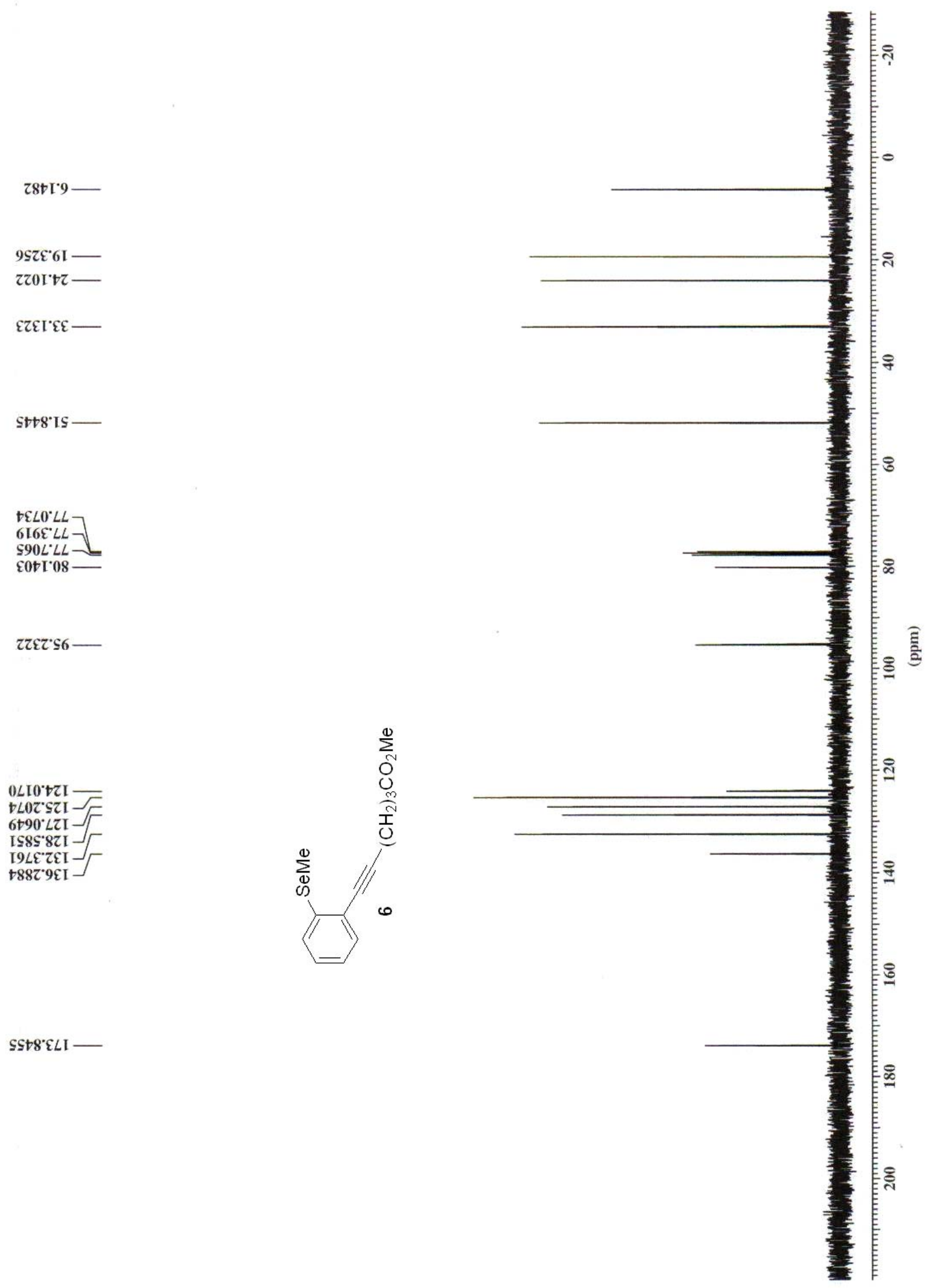
$0000^{\circ} 0$

†SE6 $6^{\circ}$

$0 \mathcal{E S} 6^{\circ} \mathrm{I}-$

$80 \angle 6^{\circ} \mathrm{I}$

S886 I

$8900 \%$

IzZE' 7

IIsS $z$

9L9S:

$t \$ 8 S^{\circ} z \sqrt{t}$

$\left[889^{\circ} \mathcal{E}\right.$

$6 \angle L 0^{\circ} L$

$+\$ 80^{\circ} \mathrm{L}$

$\varepsilon 260^{\circ} \mathrm{L}$

$6960^{\circ} \mathrm{L}$

$\$ 660^{\circ} \mathrm{L}-$

ESOI' $L-$

$660 \mathrm{I}^{\circ} \mathrm{L}$

$8 \mathrm{III}^{\circ} \mathrm{L}-$

$+6 \mathrm{II}^{\circ} \mathrm{L}$

$9881^{\circ} \mathrm{L}-$

9I6I' $L$

$880{ }^{\circ} L$

$0 Z$ I ${ }^{*} L$

StI $2 \cdot L$

Ezz: $L$

$6 \varepsilon \varepsilon Z^{\circ} L$

0E9 'L

$\checkmark 9 z \mathcal{E} L$

$6+t \varepsilon^{*} L$

$0<\vdash \mathcal{E} L$

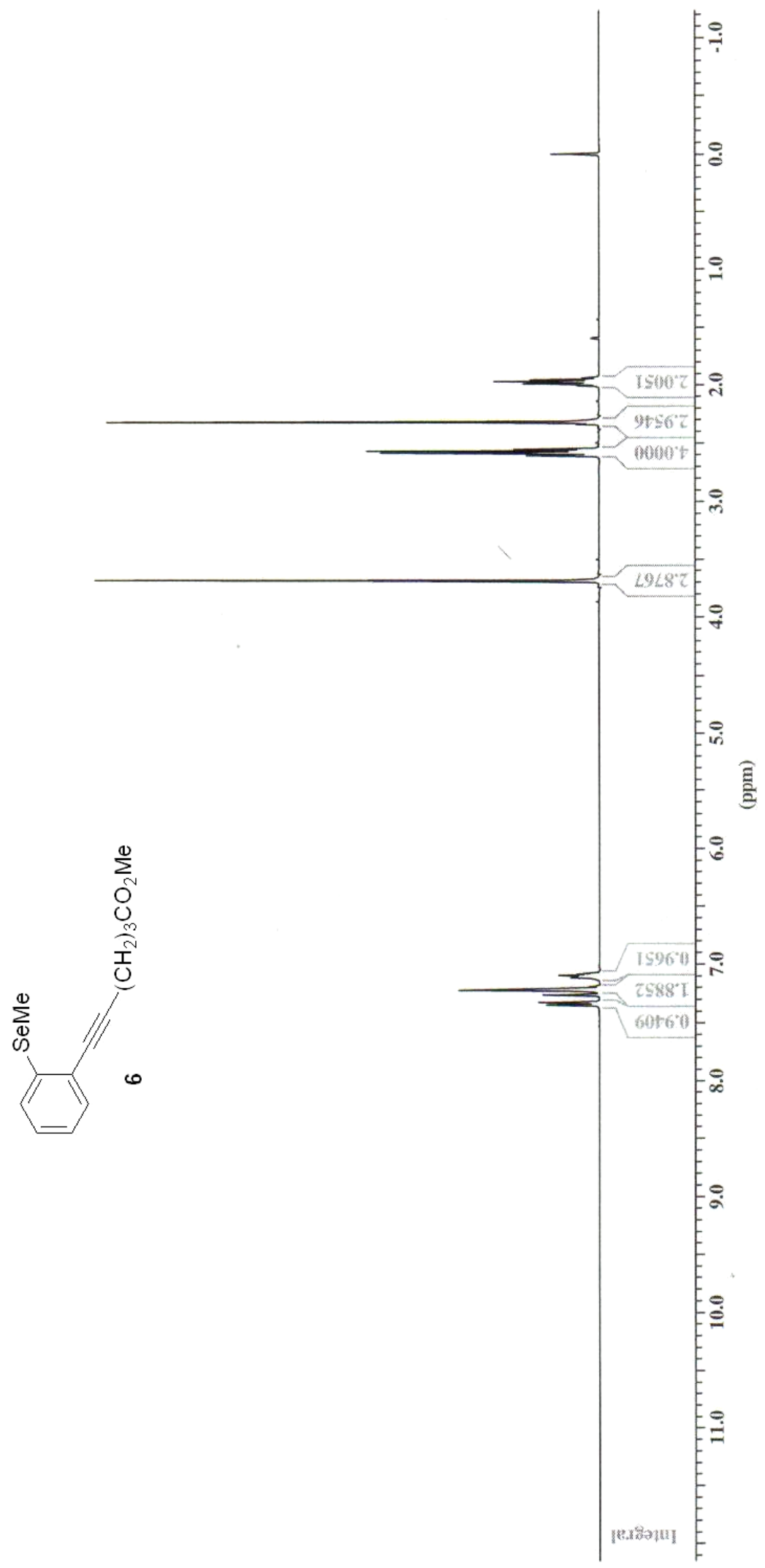


LLEI'9-

t909. IE-

012866

I $\$ 80^{\circ} \mathcal{E I}$ I E89 $\varsigma$ ZI 86806ZI]

8EZEZEI
96L9 9 LI

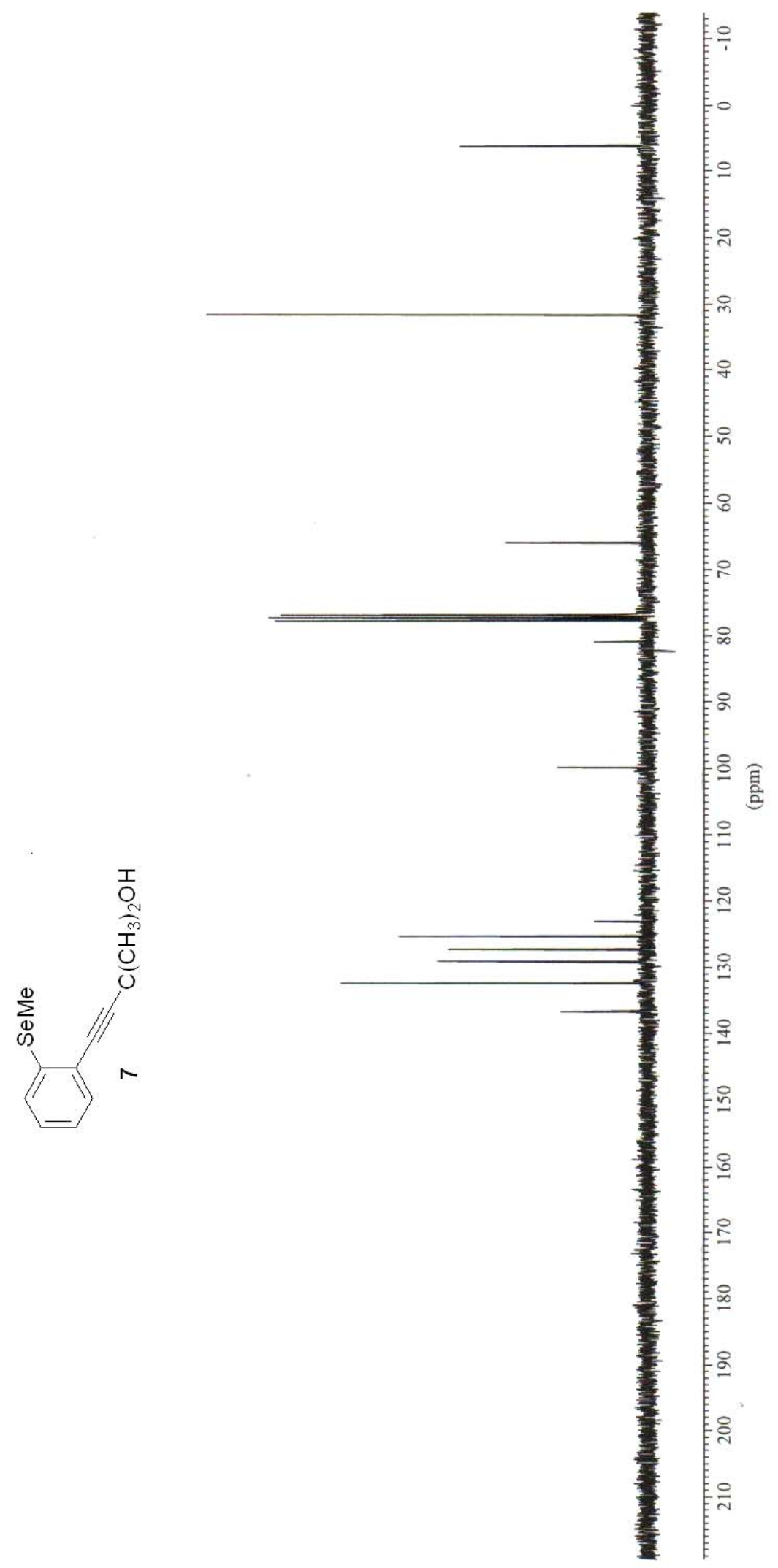




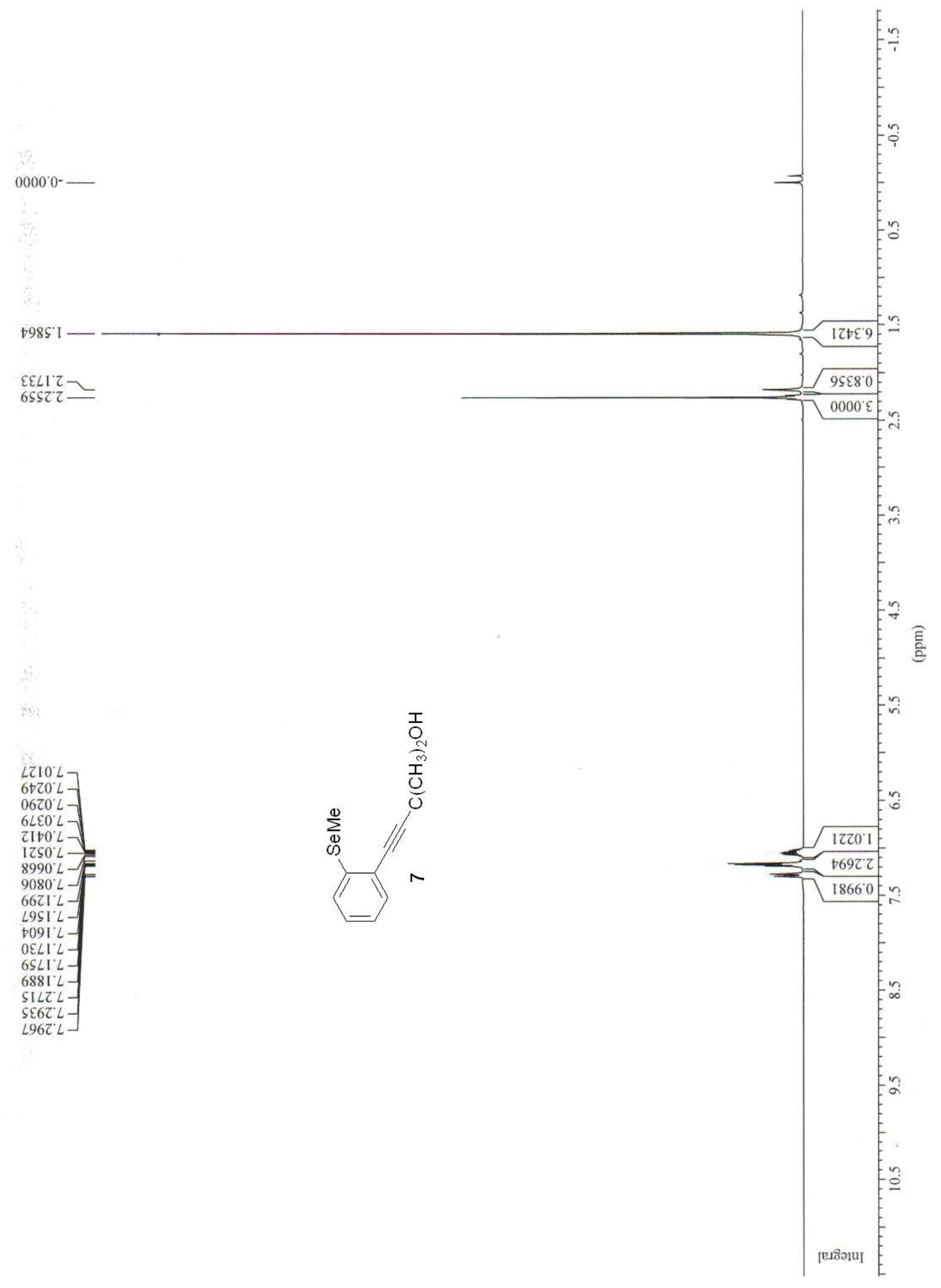



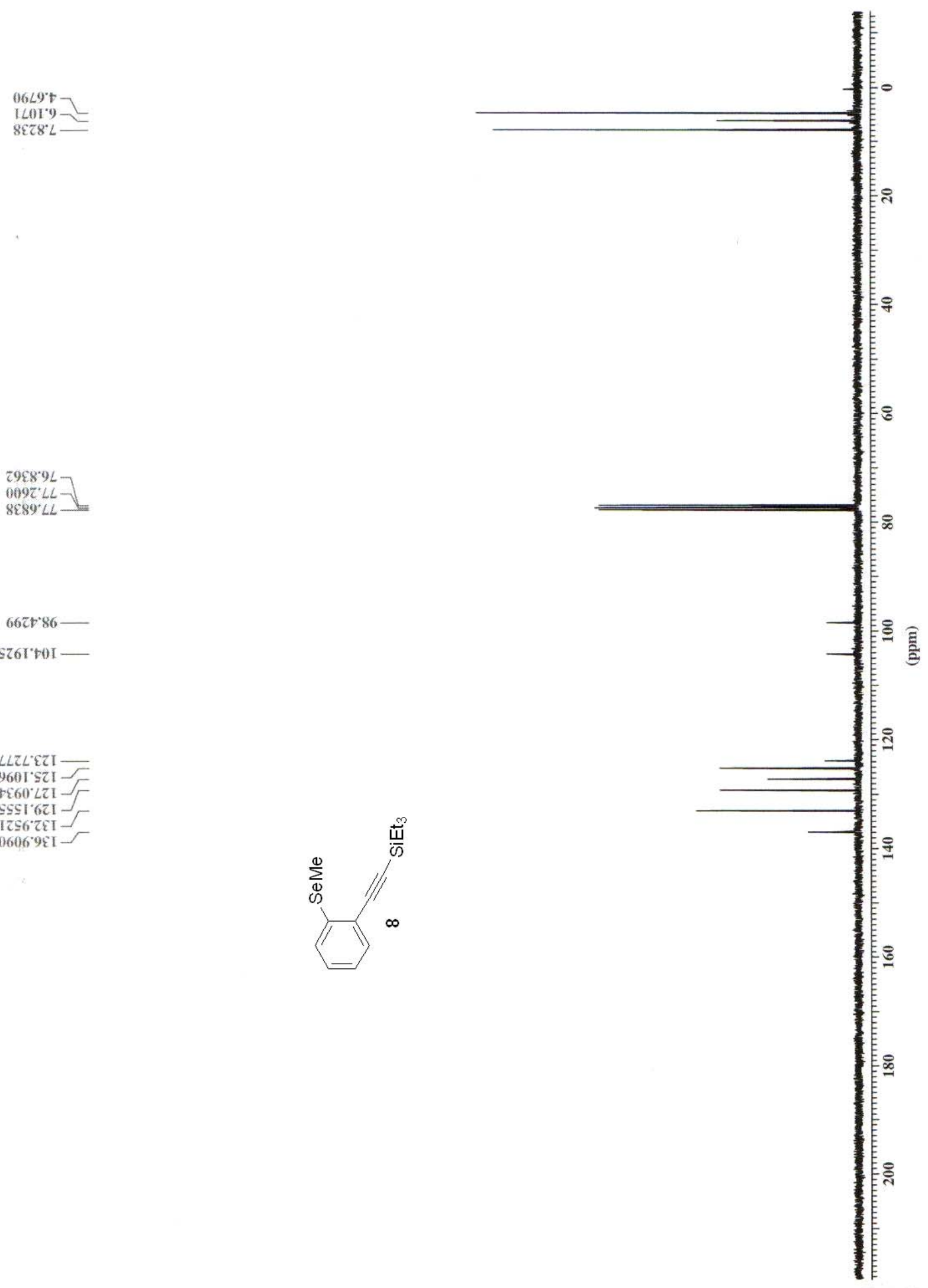

2988*9L-

$\left.8889^{\circ} \mathrm{LL}\right]$

LLZL'GZI

$960 \mathrm{I}^{\circ} \mathrm{SZI}-\mathrm{T}$

$5860^{\circ} \mathrm{LZI}-\bar{T}$

IZS6.7हI -

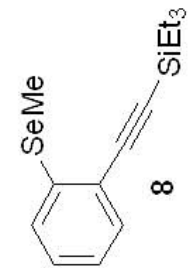




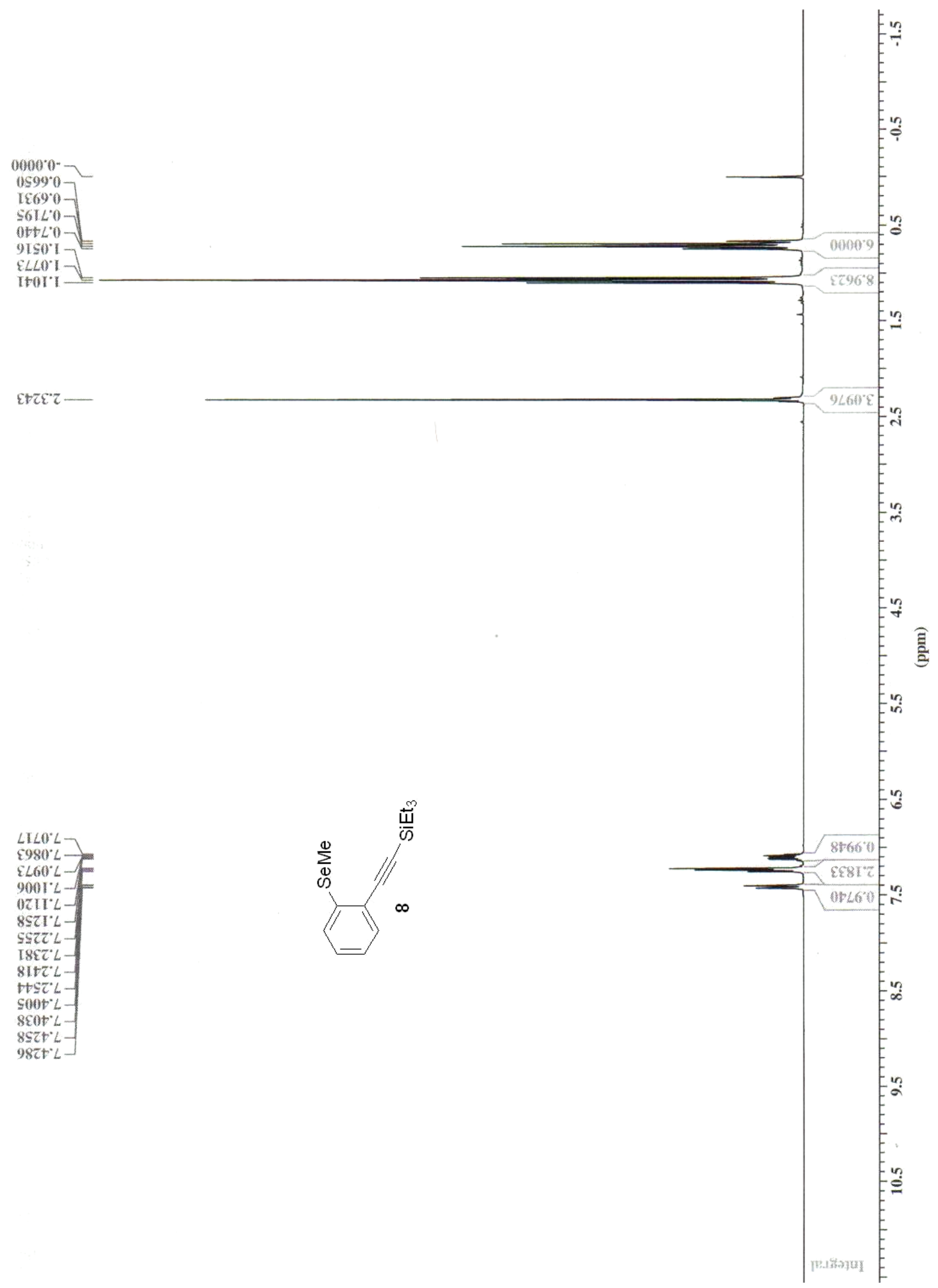




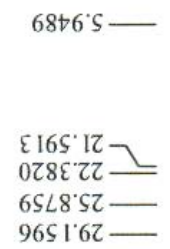

$2908^{\circ} 9 \mathrm{~L}$

$00 \varepsilon Z^{\circ} L L$
$8 \varepsilon S 9^{\circ} L L$

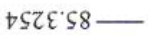

It9 $9 \div 26$

IEILOOZI-

$\varepsilon S Z 0^{\circ} \rightarrow Z 1-$

ऽ 50 LZI-

†0LE' 821$]$

LS88 IEI I I

EZ0Z $9 \varepsilon \mathrm{I}-$
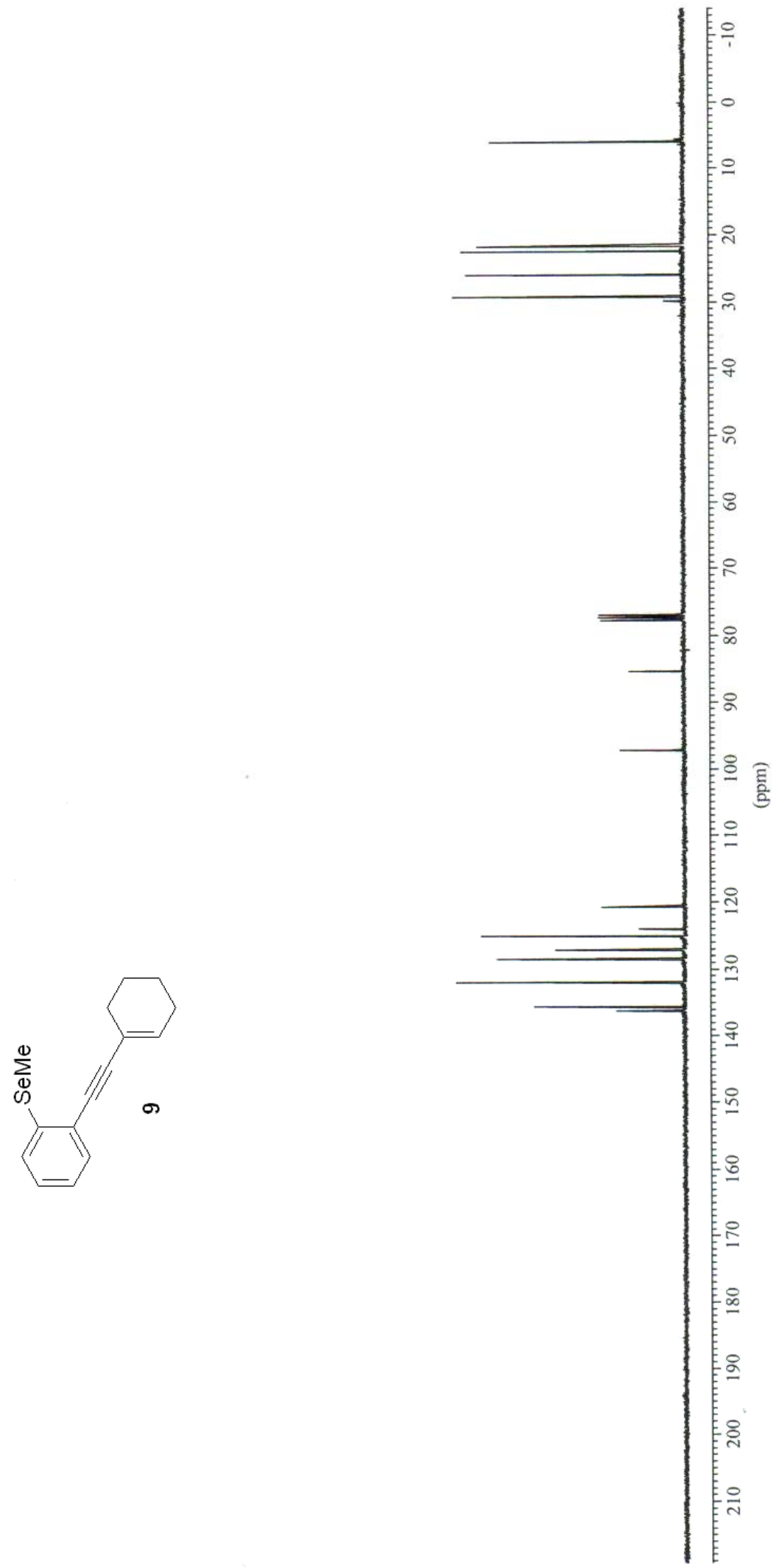


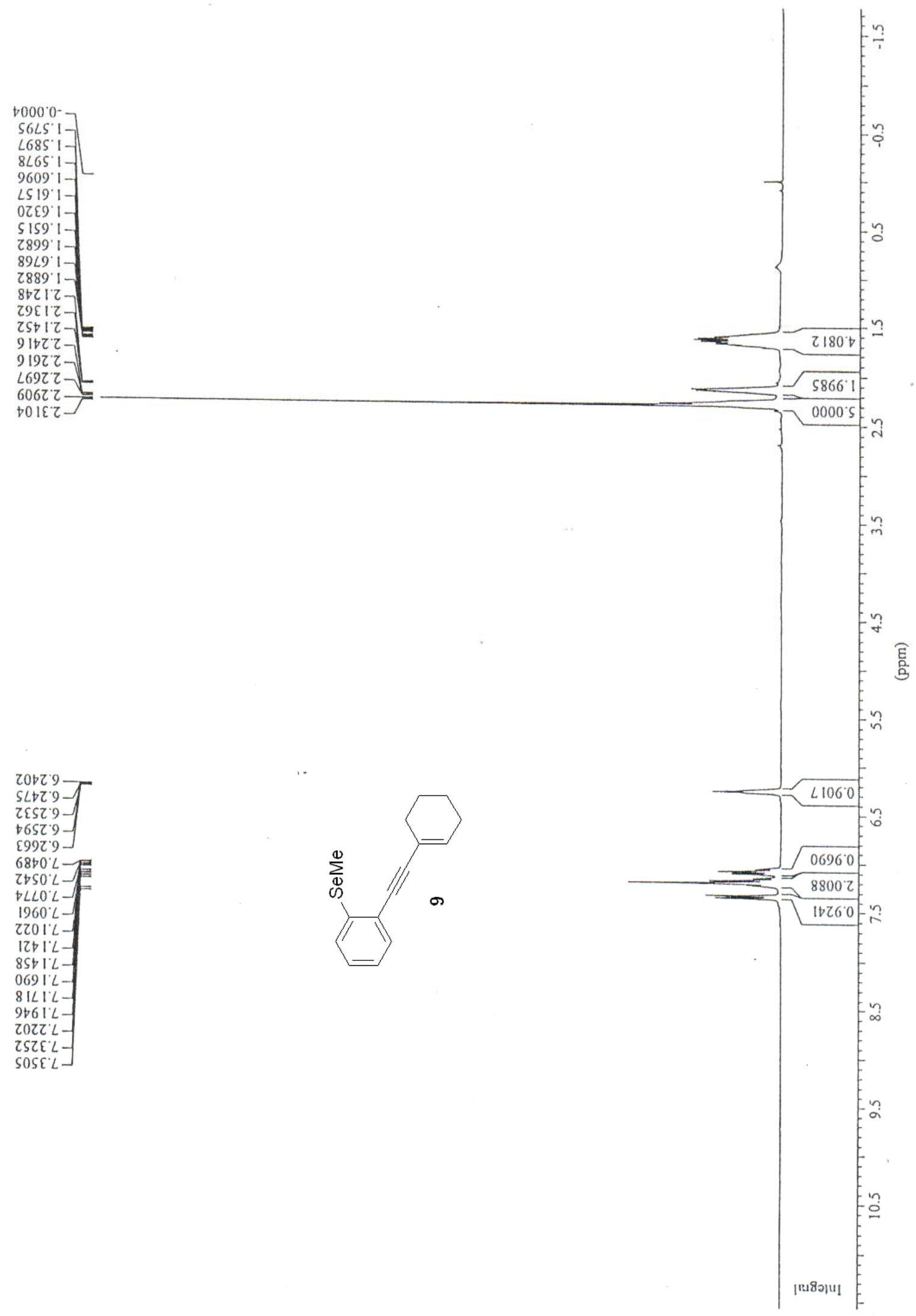




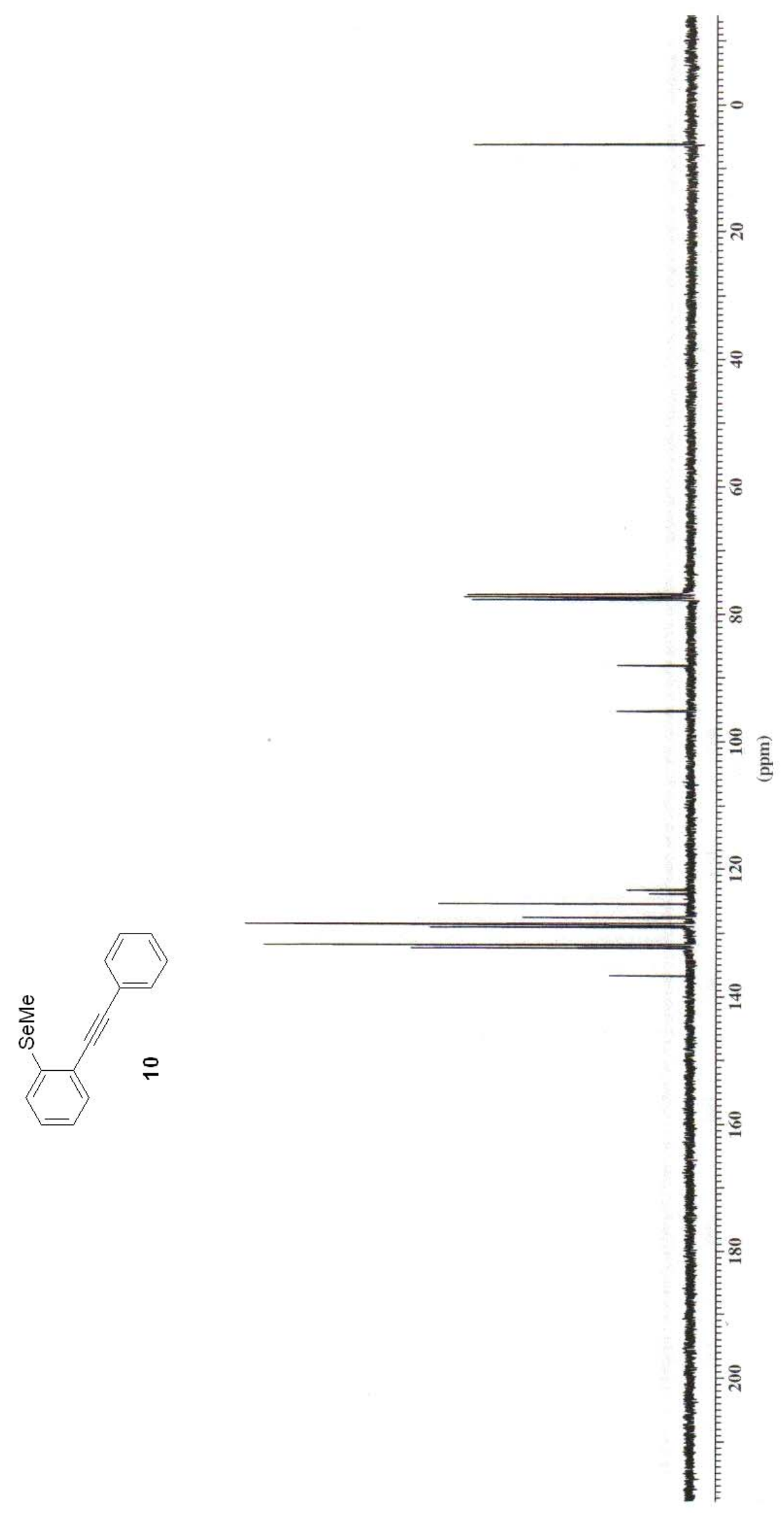

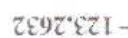

09LL'EZI L

I896 $S 21-$

Z6IS"LZI-F

Etes.8zI

IIt09.821-

$\angle 810^{\circ} 621$ -

LILL'IET -

9ItE' 2 \& 
$0000^{\circ} 0^{\circ}$

$s+2 s 1$

$\operatorname{trse} \tau$

tser:

Stri: $L$

$\cos 1 \mathrm{~L}$

$\angle L 9 \mathrm{~T}^{\circ} \mathrm{L}$

TELI'L

cozz'L

SSEZL

GET:L

$\angle 95{ }^{\circ} L$

II $\angle Z^{*} L-1$

$820 \varepsilon^{\circ} L-$

$\angle T E Q L]$

ESE ${ }^{\circ} L$

$085 E^{\prime} L-$

ESSE $L$

IS9E $L-$

ILSt $L-$

E285 $L$

I29S: $L$

$569 \mathrm{~s}^{\circ} \mathrm{L}$

t085: $L$

$8 \mathcal{E} 6 \mathrm{~S}^{\circ} \mathrm{L}-$

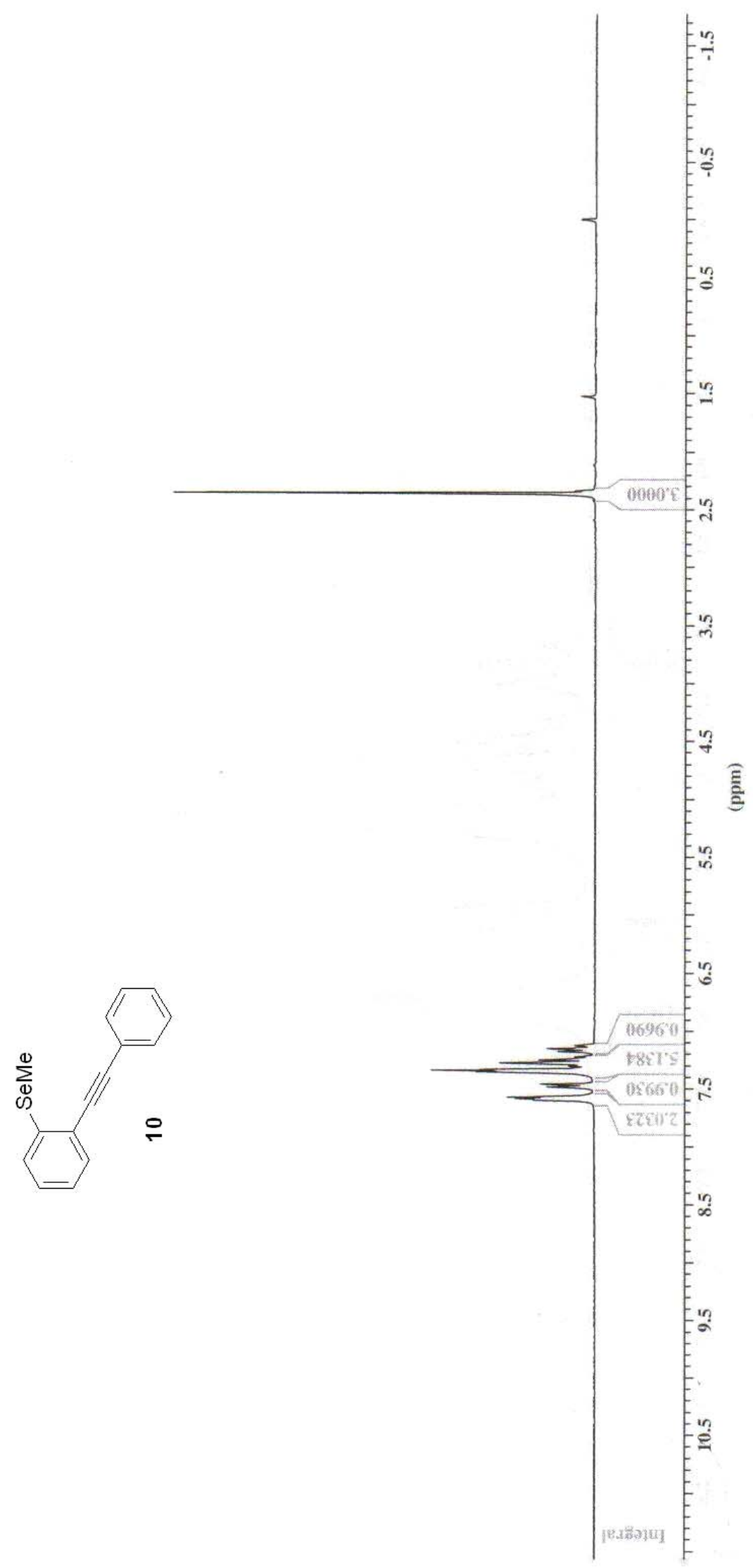




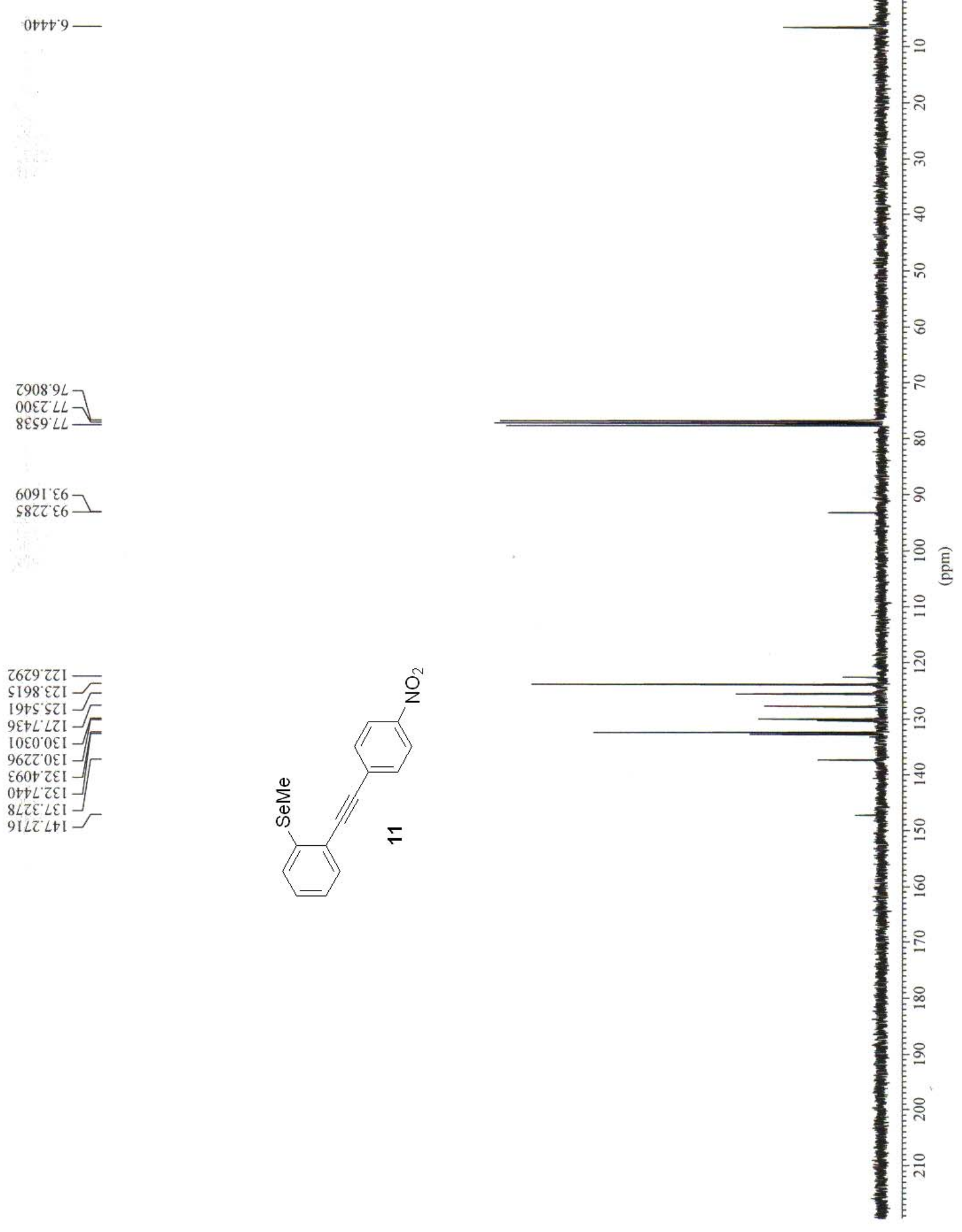


$\angle 000^{\circ} 0^{-}-$

ว£6ะ

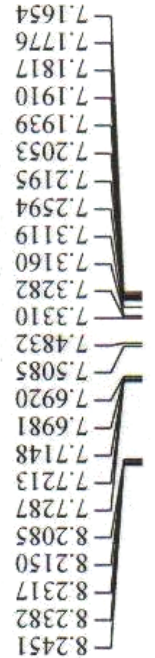

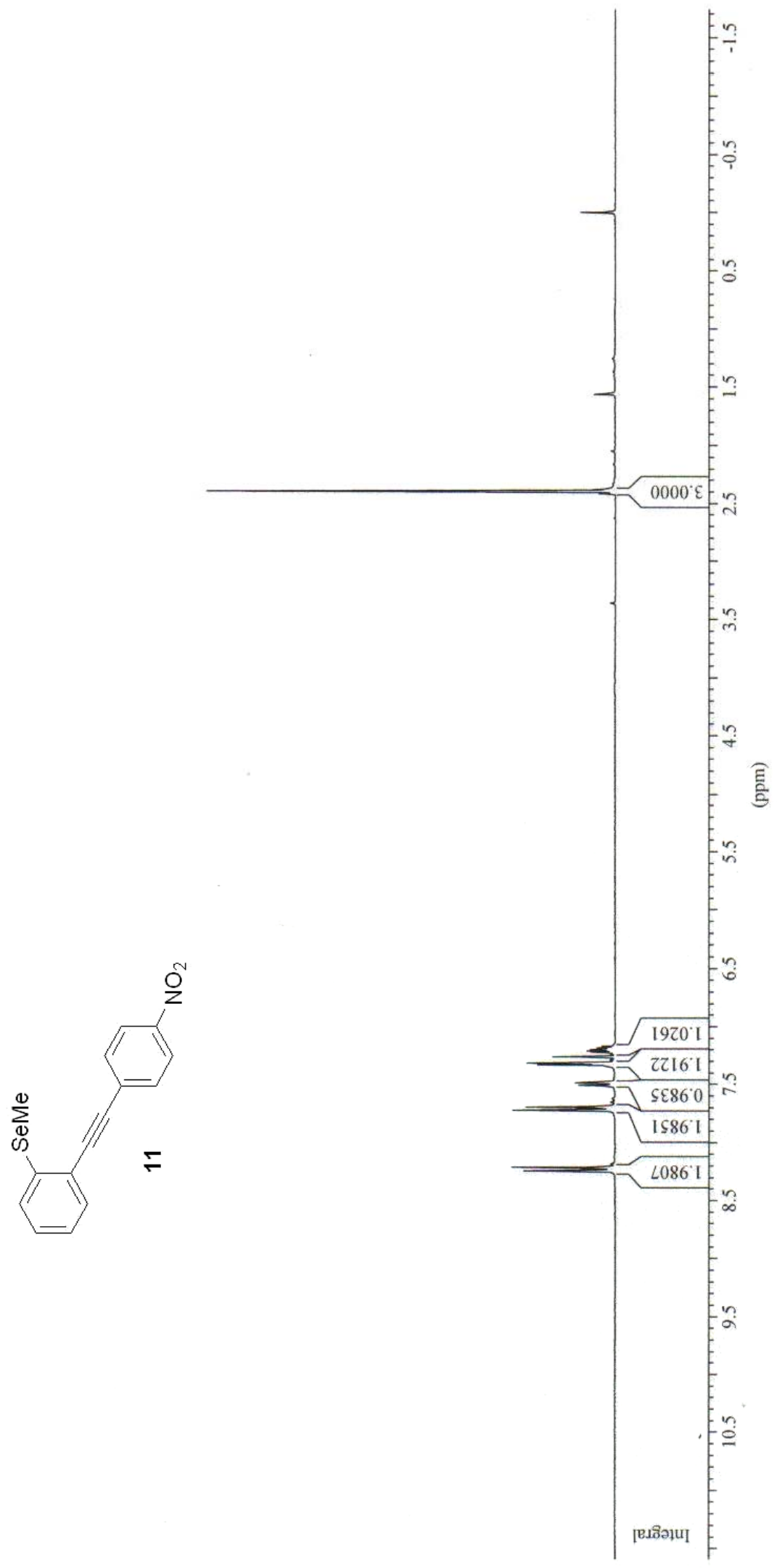



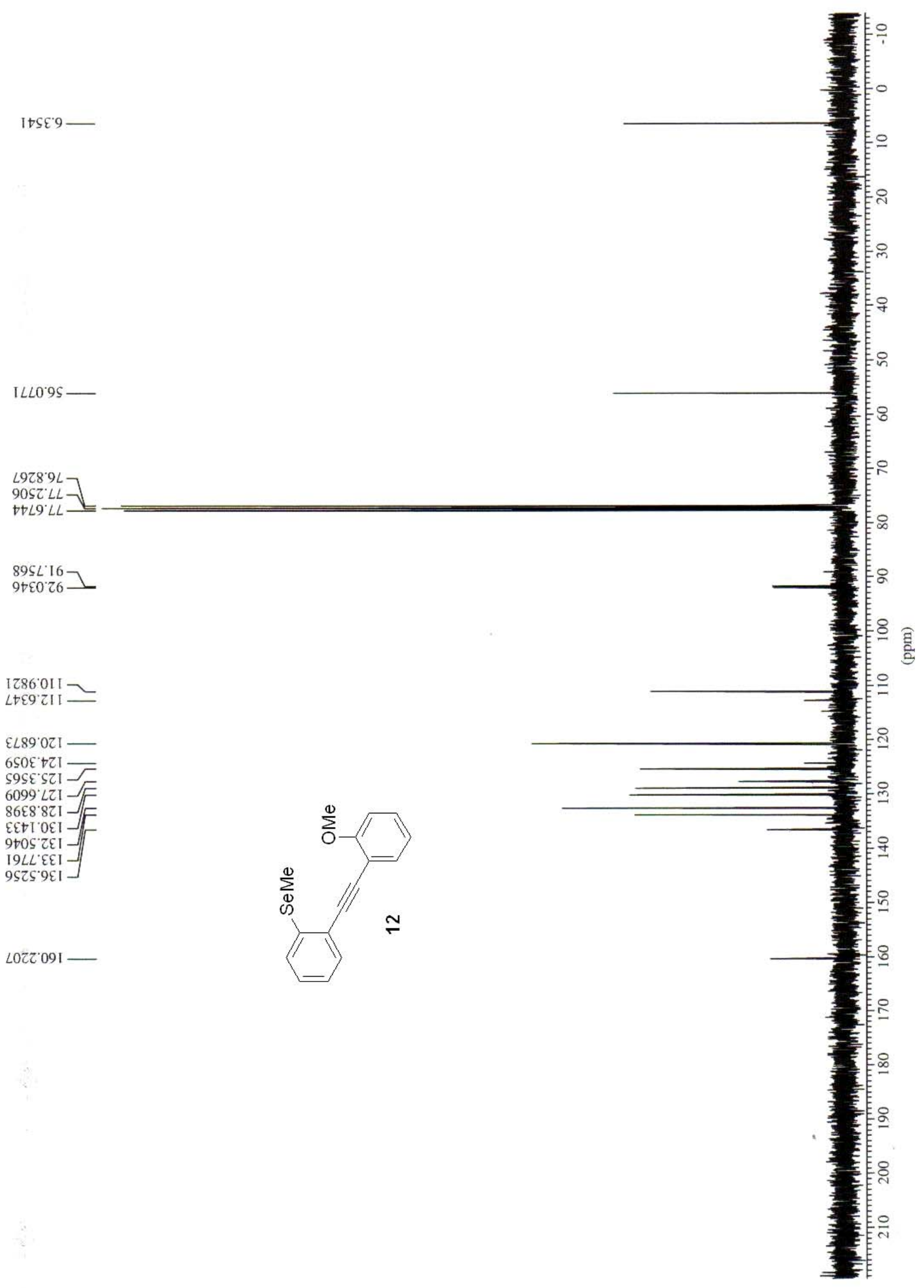


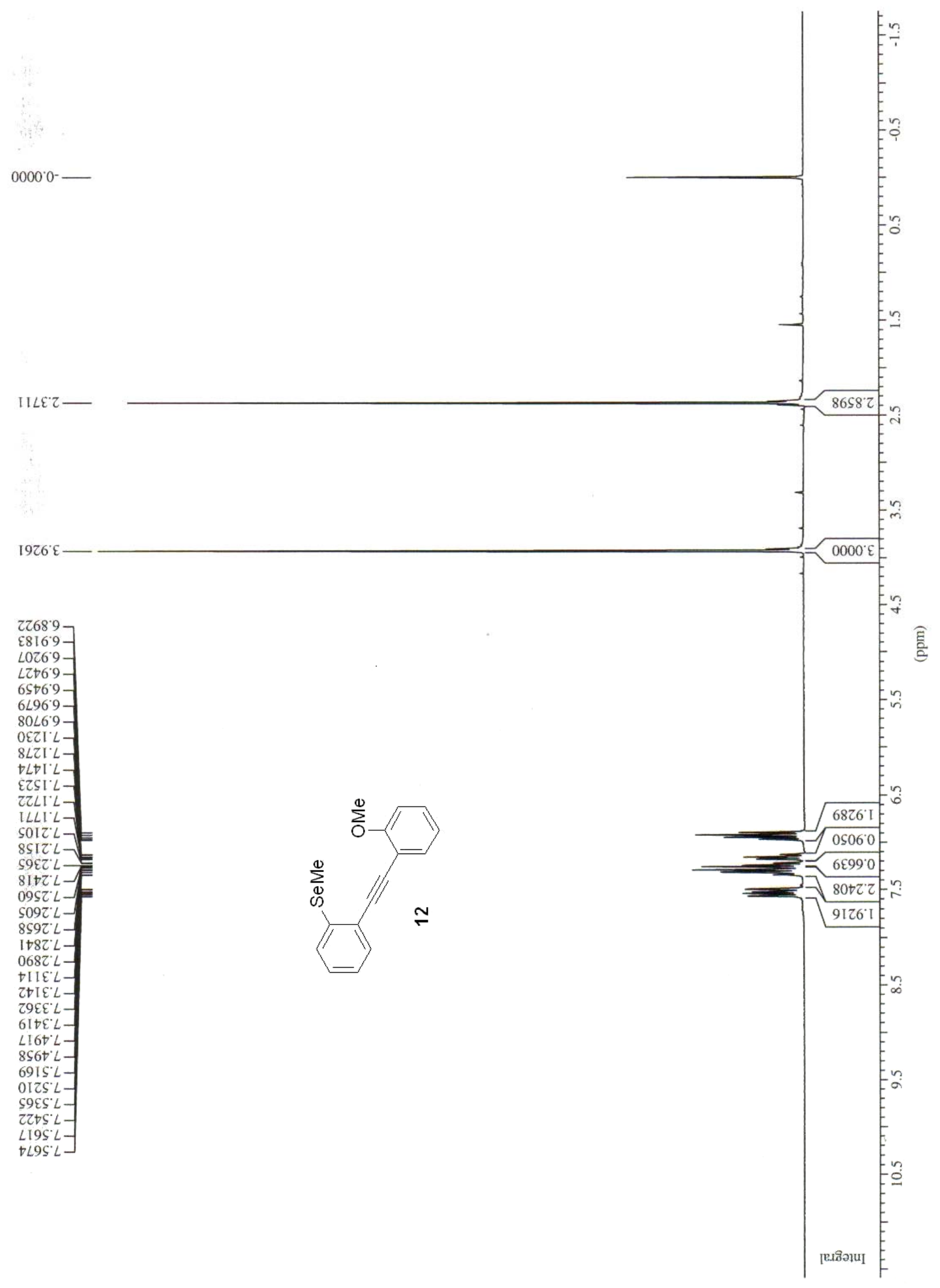


$\Im S \angle \varepsilon^{\prime} 9$

8ย\{8 $9 L$

$\angle L S C^{\circ} L L$
S189.LL

$\varepsilon \varsigma 96.68-$

$0086 \sqcup 6$

$8 \varsigma 1 \varepsilon \varepsilon Z 1-$
$+119 \varepsilon Z 1-$

0L9t'sZ1]

6SE9 $\angle Z I-$
$\angle 0 L Z 6 Z 1-$

Z8ZL IEI-

619t'ZEI-

LLI8.9 1$]$
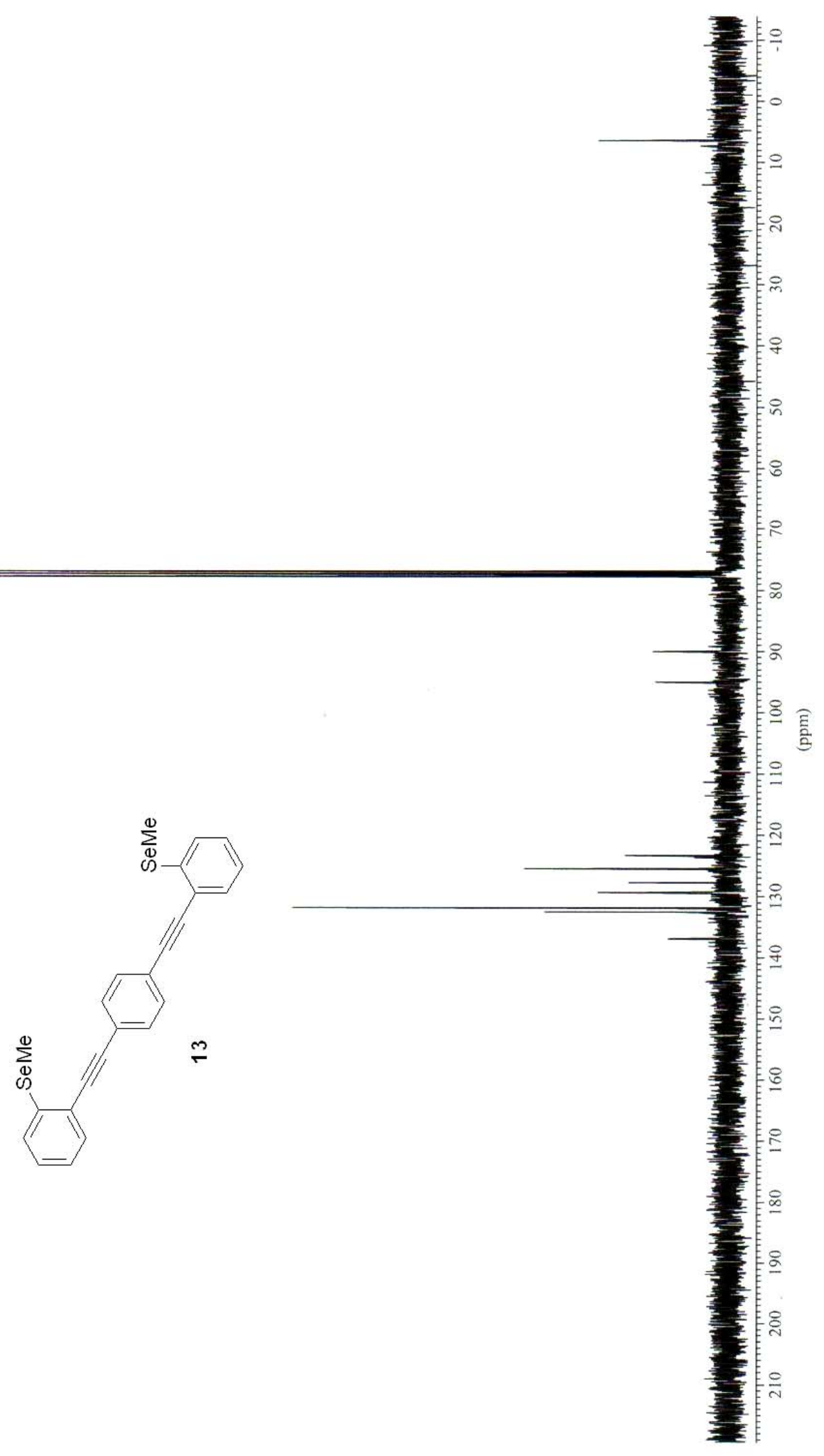


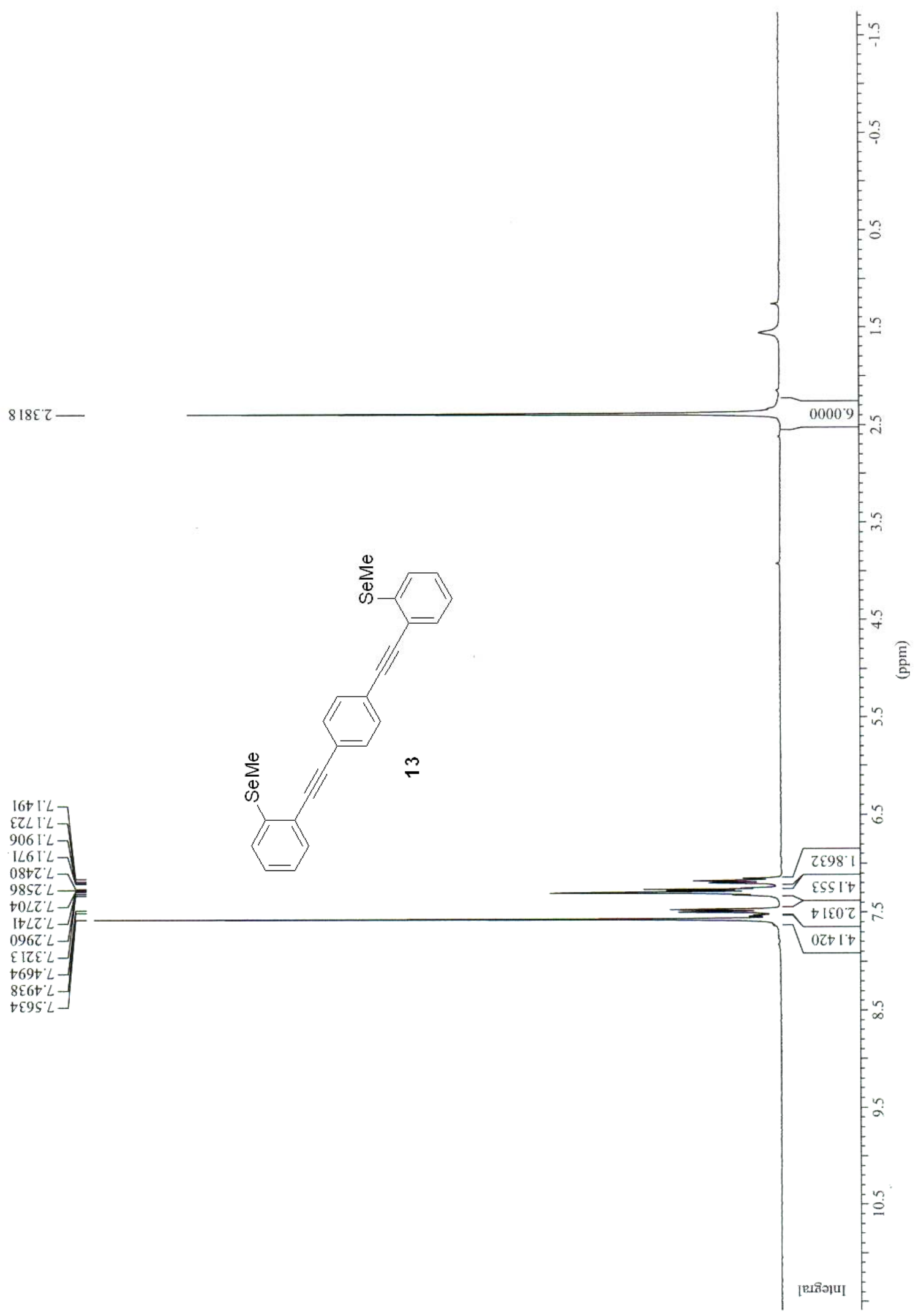




$$
\text { A }
$$




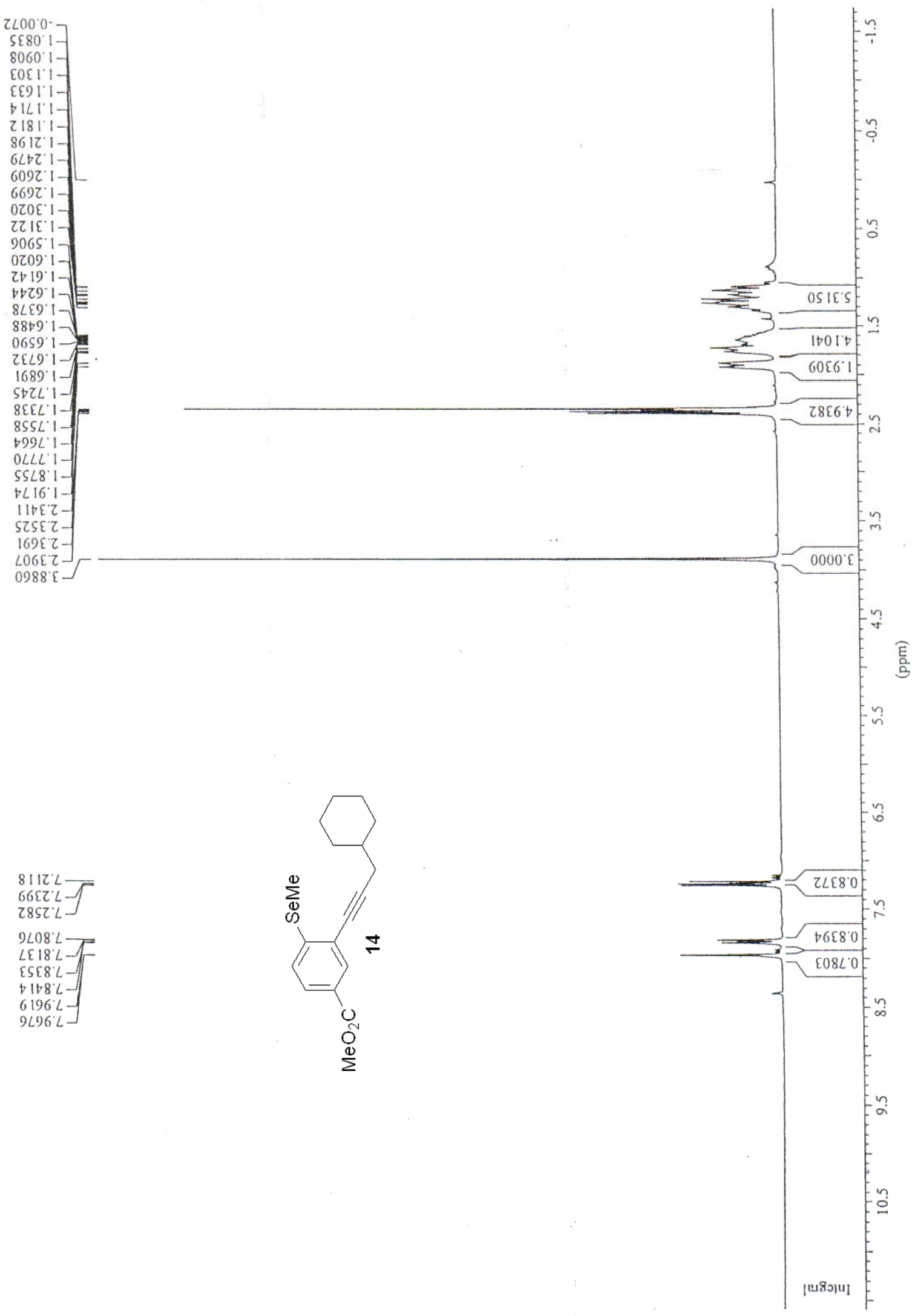


Eq0t't -

6\&t6 $2 Z$

$180 t^{\circ} 6 z$

$\angle 89 t^{\circ} 6 z$

0989.62

S86S'IE-

9ธזा'ze

$99+\Gamma^{\circ} 9 \mathcal{E}$

E298.9L

z982 LL

$00 I L L^{\circ} L L$

26It'88-

z0Iร $S$ TI

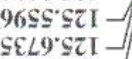

$0 z 8 \angle L Z \mathrm{I}$

69tI'6e I -

s8zz' $\mathrm{EtI}$

IEtz's+I -

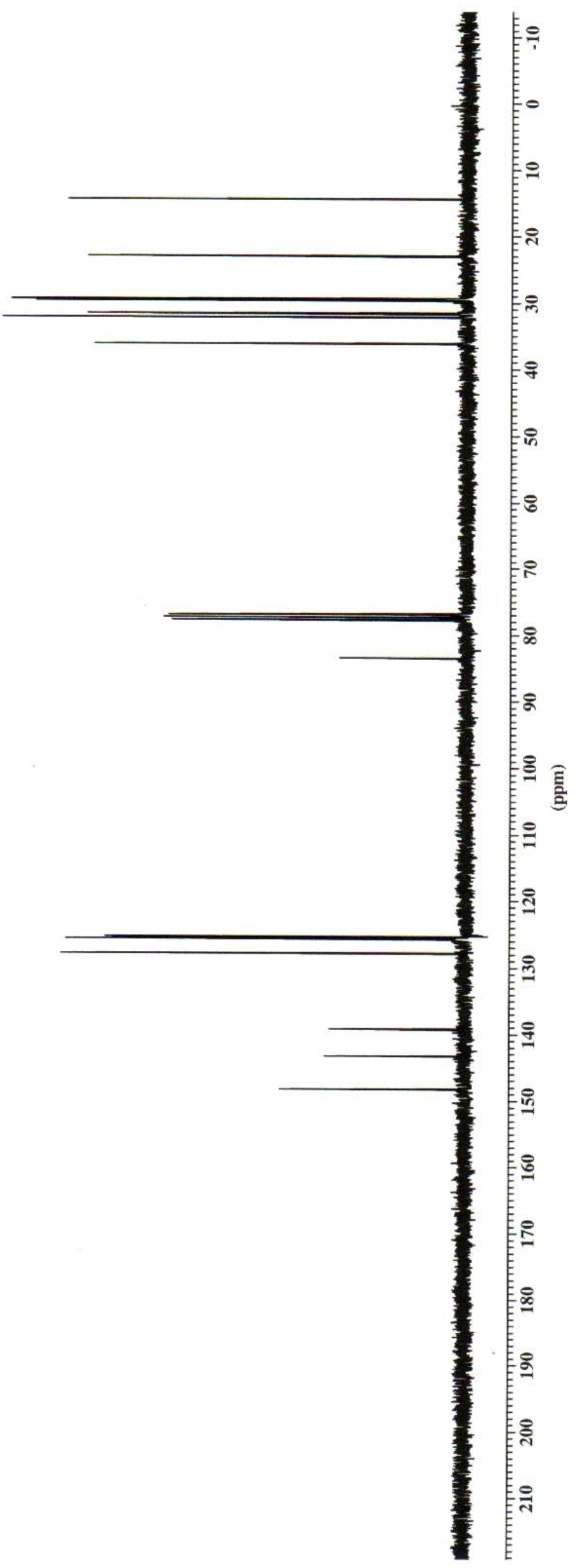




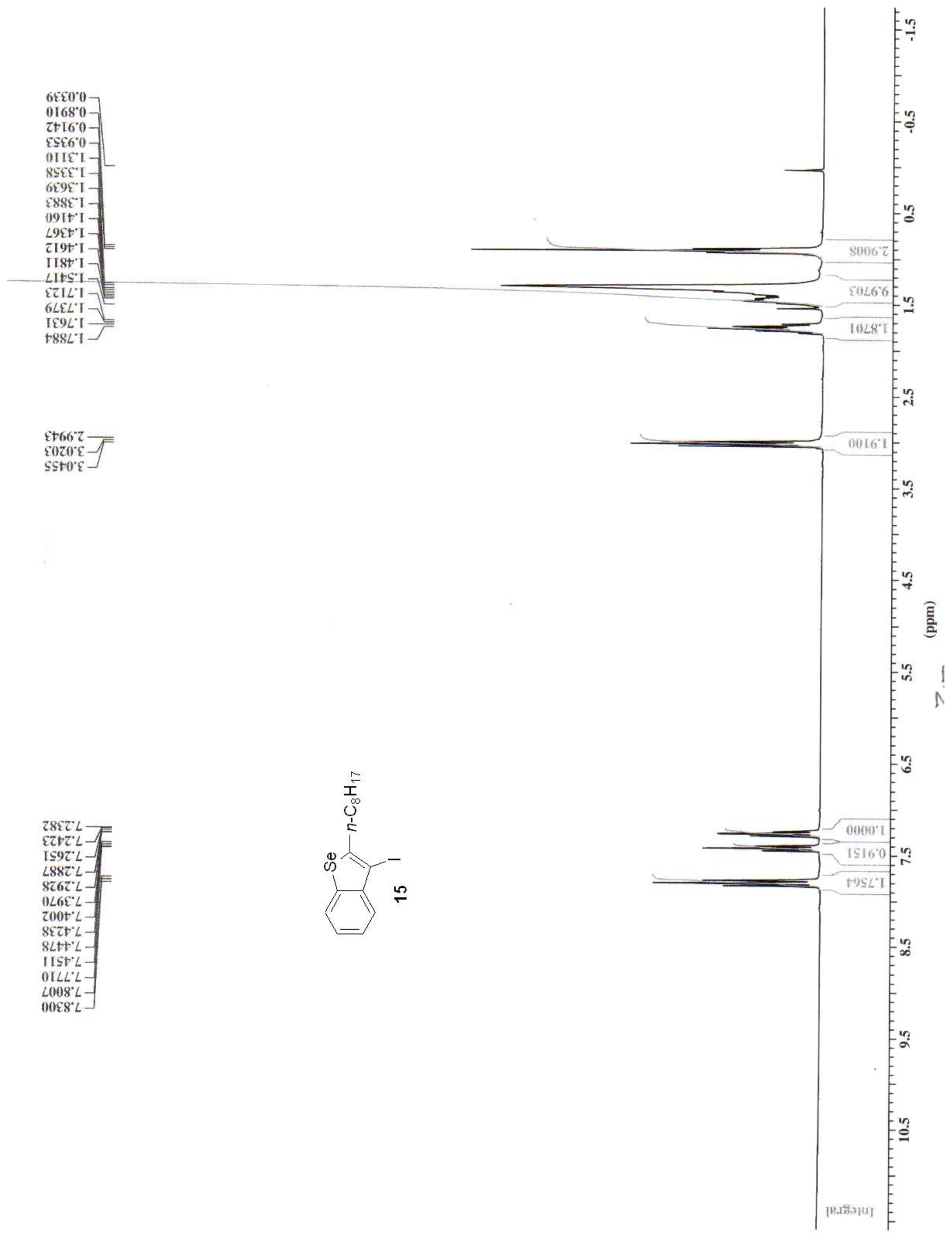


7908.9L-

$00 E T^{\circ} L L-$

$8 \mathcal{8} S 9^{\circ} \angle L-$

$\varepsilon 9+6 \div 8$

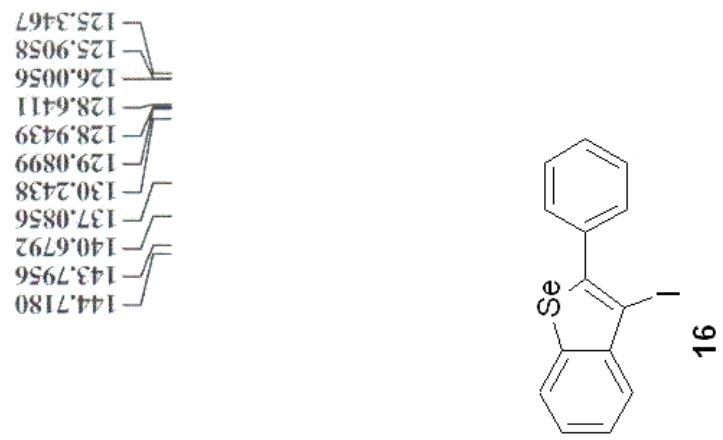

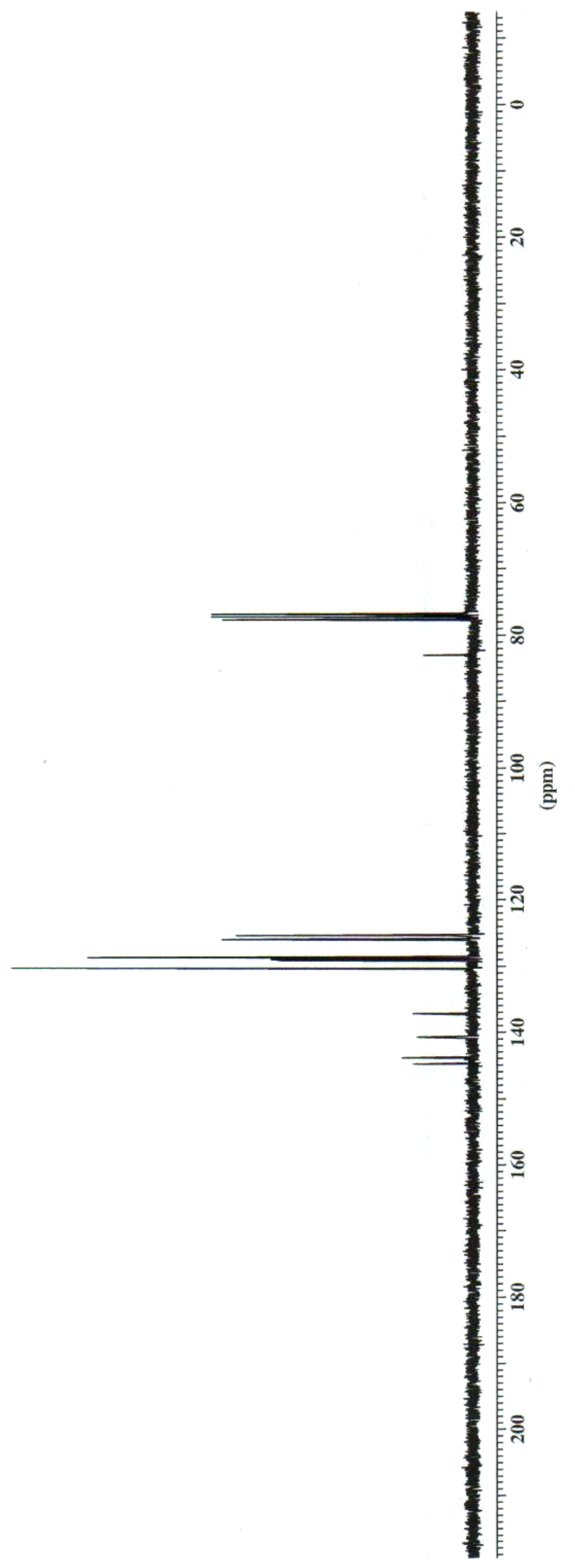


$0000^{\circ} 0^{\circ}-$

8ses I -

$\varepsilon I S \tau: L-$

6SIE'L-

$Z \operatorname{tEE} L$

$9 E S E^{\circ} L$

I0It"L-

ILZT: L

IStT $L$

$L L+T^{\circ} L$

$0 \varepsilon 95: L$

ES9T: L-

$0280^{\circ} L-F$

$6909^{\circ} \mathrm{L}-$

I0I0 $\mathrm{L}-$

$1929^{\circ} \mathrm{L}-$

$0828^{\circ} L$

$9 L+88^{\circ} L-$

$\angle 2 \pi 6^{\circ} \mathrm{L}-$

$82+6.2]$

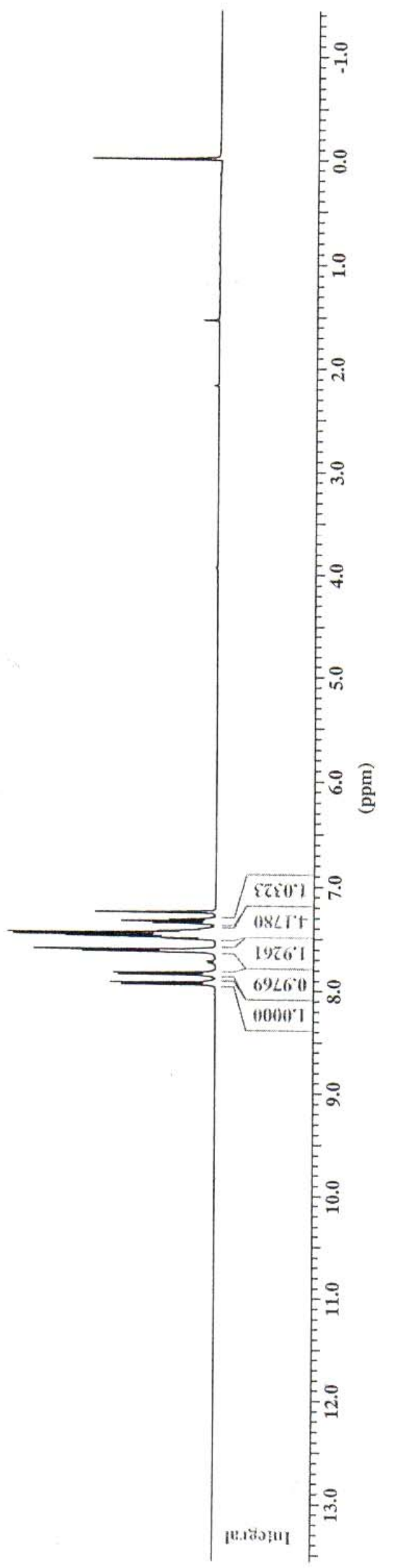


\&S96.IZ-

$\mathcal{E S} 00^{\circ} \mathcal{E Z}$

L6LL'SZ

\$86 $S^{\circ} 0 \varepsilon$

Z908.9L

$00 E Z^{\circ} \angle L$

$8 E S 9^{\circ} L L$

SEL8 08

L8LE'SZI

8L9t'S2I

¿IEL" $\$ Z I] / F$

$\varepsilon+z$ I"z I-

[S89.t\&I]

SLOS'6EI

ZLStEET

$89 \angle 6 \angle L I$

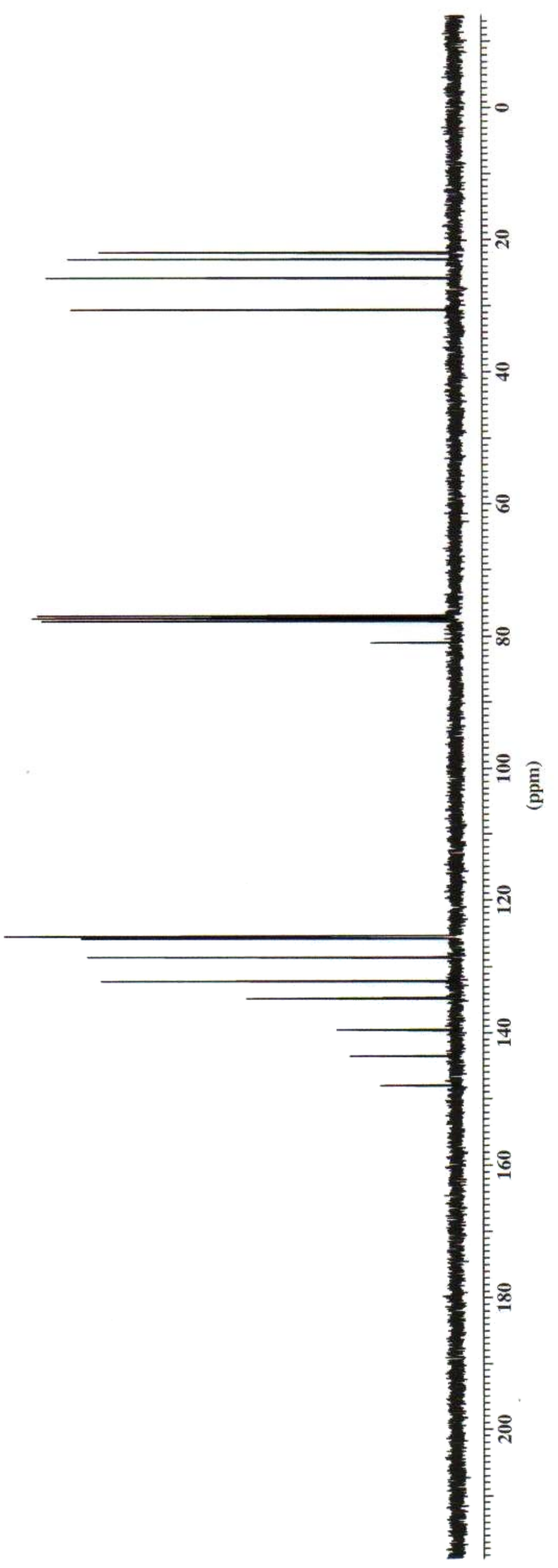



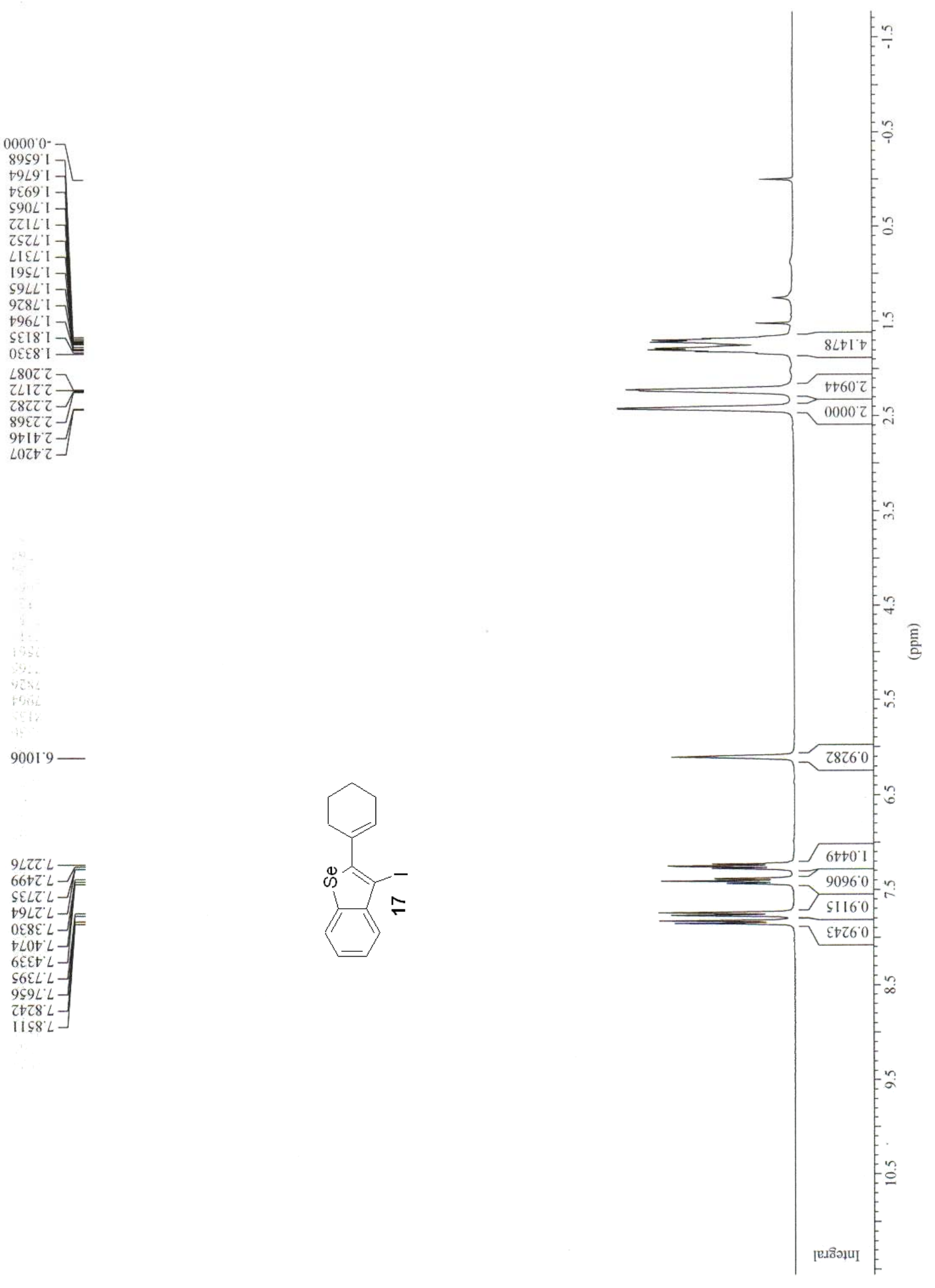

$9 \angle 2 Z L$

$66+2 \angle L-F$

$S E L Z L L$

D9LZL

OE8E $L$

$\forall L 0 t L-$

S6E $L^{\circ} L$

$959 L$

$2+28^{\circ} \mathrm{L}$

II $\mathrm{CP}^{\circ} \mathrm{L}$

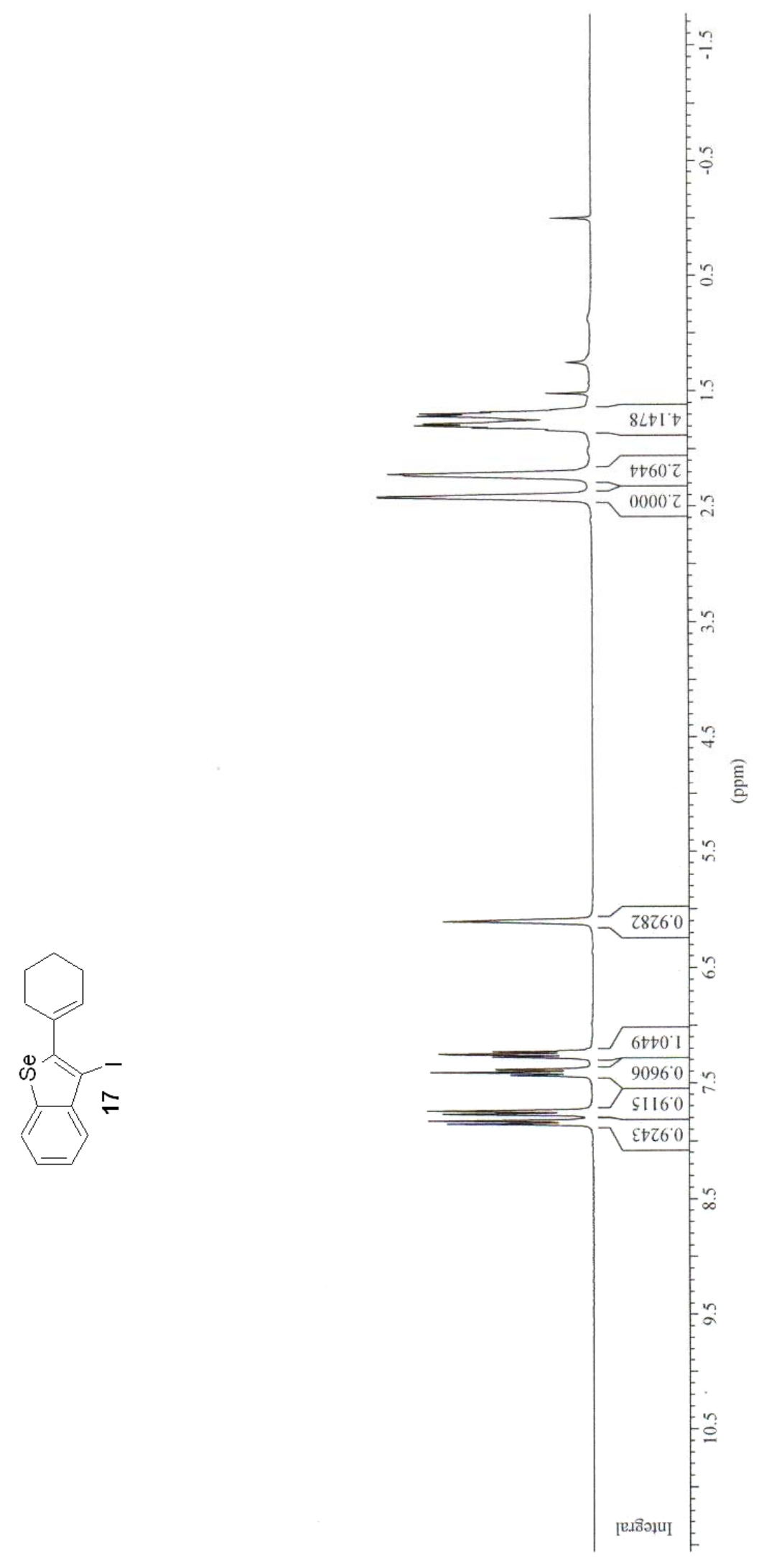

$9001^{\circ} 9$ 


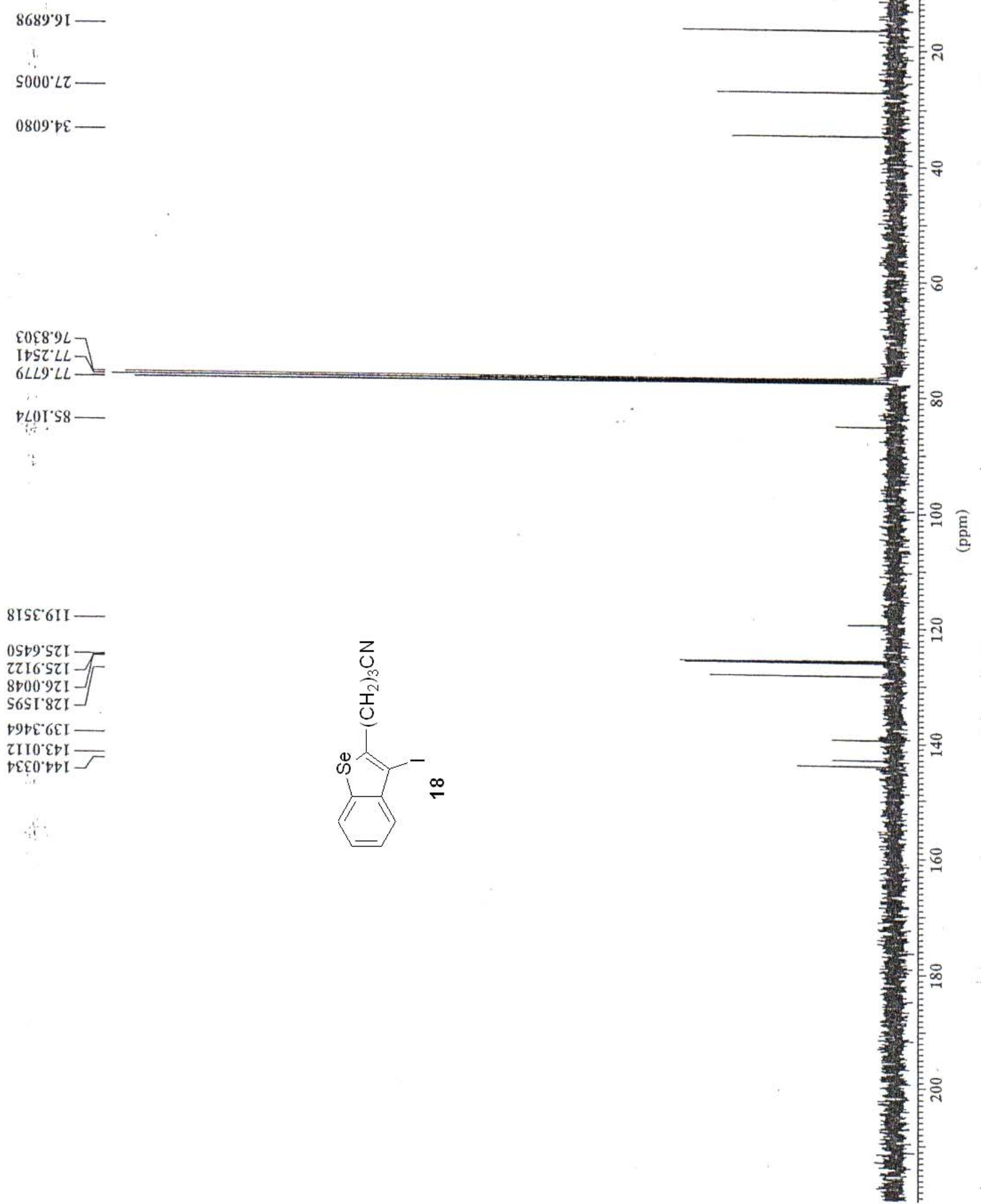


$0000^{\circ} 0^{-}$

$\angle Z L 0{ }^{\circ} Z$

$2 \angle 60 \tau$

91212

t0LL' $Z$

zISt $z$

$\varepsilon S \angle t=$

E66t"

$8 \mathrm{I}^{\circ} \mathrm{I}^{\circ} \mathcal{\varepsilon}$

$\angle 98 I^{\circ} \varepsilon-$

IIIZ' $\varepsilon$

LST' 6LIT. 9SLI'L]

$9662^{\circ} L$

$\nabla z z \varepsilon$

$09 z \varepsilon " L$

toIt

9LIt'L-

ZItt"L-

$9 S 9 t^{\circ} L$

I89t' $L$

$98 \angle L^{\circ} L-$

$2008^{\circ} \mathrm{L}$

$8 \mathcal{E Z 8 ^ { \circ } L}$

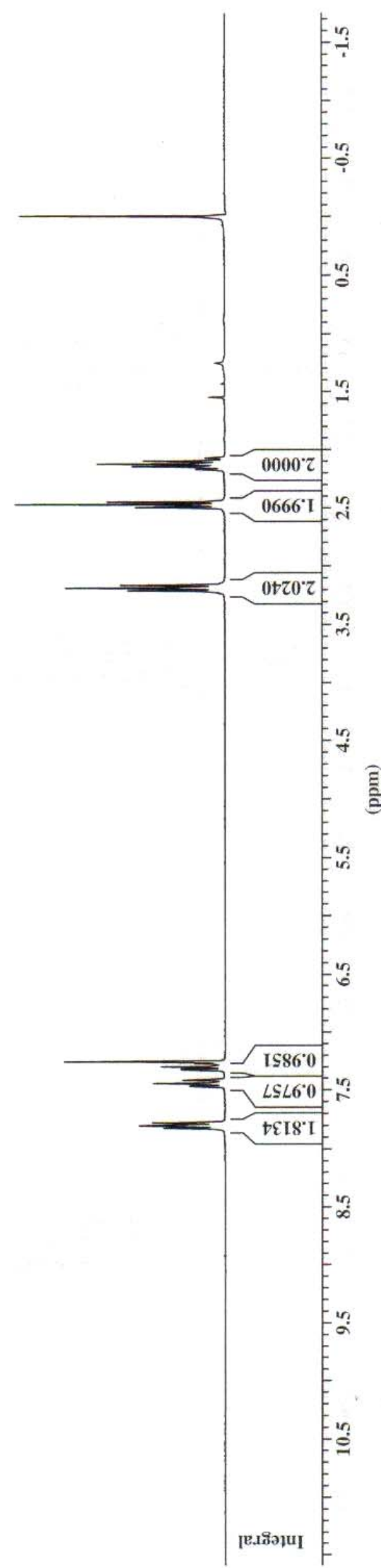


$8925^{\circ} 92$

L6ZZ'E E

E90Z'SE—

$\varepsilon \nabla 26.15$

$1878^{\circ} 9 L$

6ILZLL

$\varsigma \varsigma \triangleright \tau ' t 8$

$\nabla$ ZSৎ SZI

2S6S.SZI

$708 \mathrm{~L}$ 'SZI

1096 LZI

$8 \varsigma 8 z^{\circ} 6 \varepsilon$

$\angle 960 \mathrm{Ct}$

t08E 9 t I

[699 $\mathcal{E} L I$

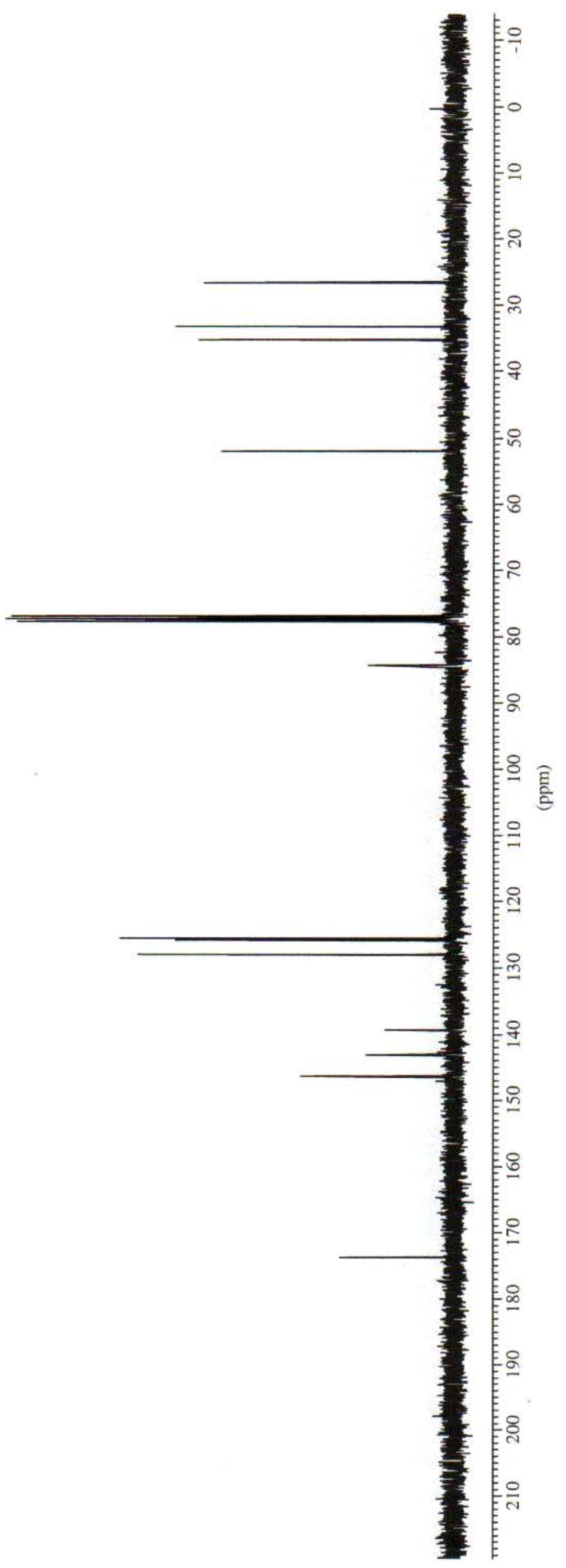


00000

$9 \angle 20 Z-7$
$+Z S 0^{\circ} Z-$

ZLLO'

SZOI'

$\varepsilon \angle Z I^{\prime} z-$

StSt 2

68LtZ L

$66+0^{\circ} \varepsilon-7$

$6 \varepsilon \angle 0^{\circ} \varepsilon-$

$000 \mathrm{I}^{\circ} \mathcal{E}$

$\varepsilon 169^{\circ} \varepsilon-$

I $9 \varepsilon \tau: L$

$\checkmark 6 \varepsilon Z^{\circ} L$

$9 \varepsilon \subseteq Z^{\prime} L-$

I09Z $L$

$998 \mathrm{Z} 2$

$6 S 8 \varepsilon^{\circ} L$

I $68 \varepsilon^{\circ} \mathrm{L}$

EOIt'L

LZITLL-

ZLED $L-$

6LSL'L-

T6LLL-

LCRLL-

$2+08^{\circ} L$

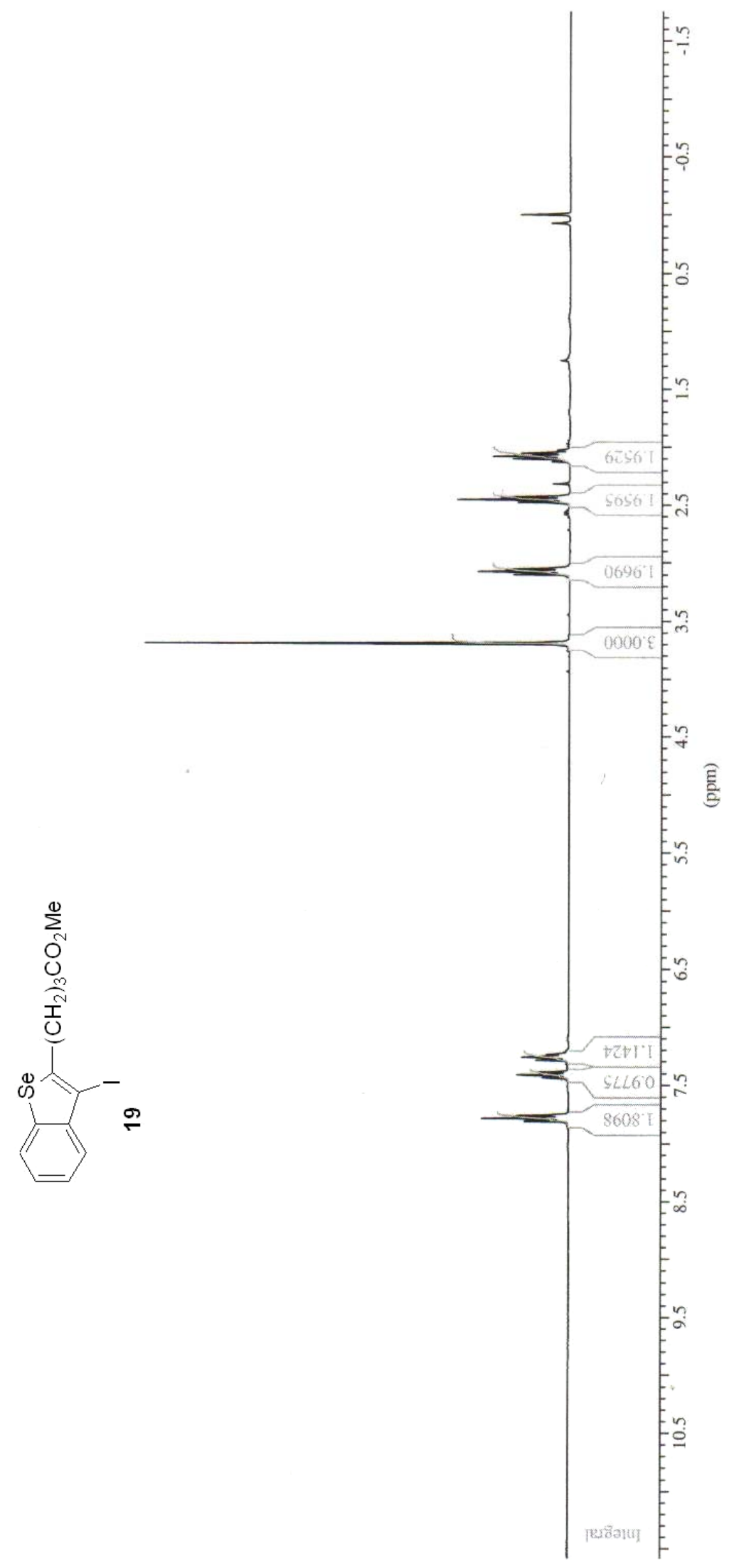

S53 

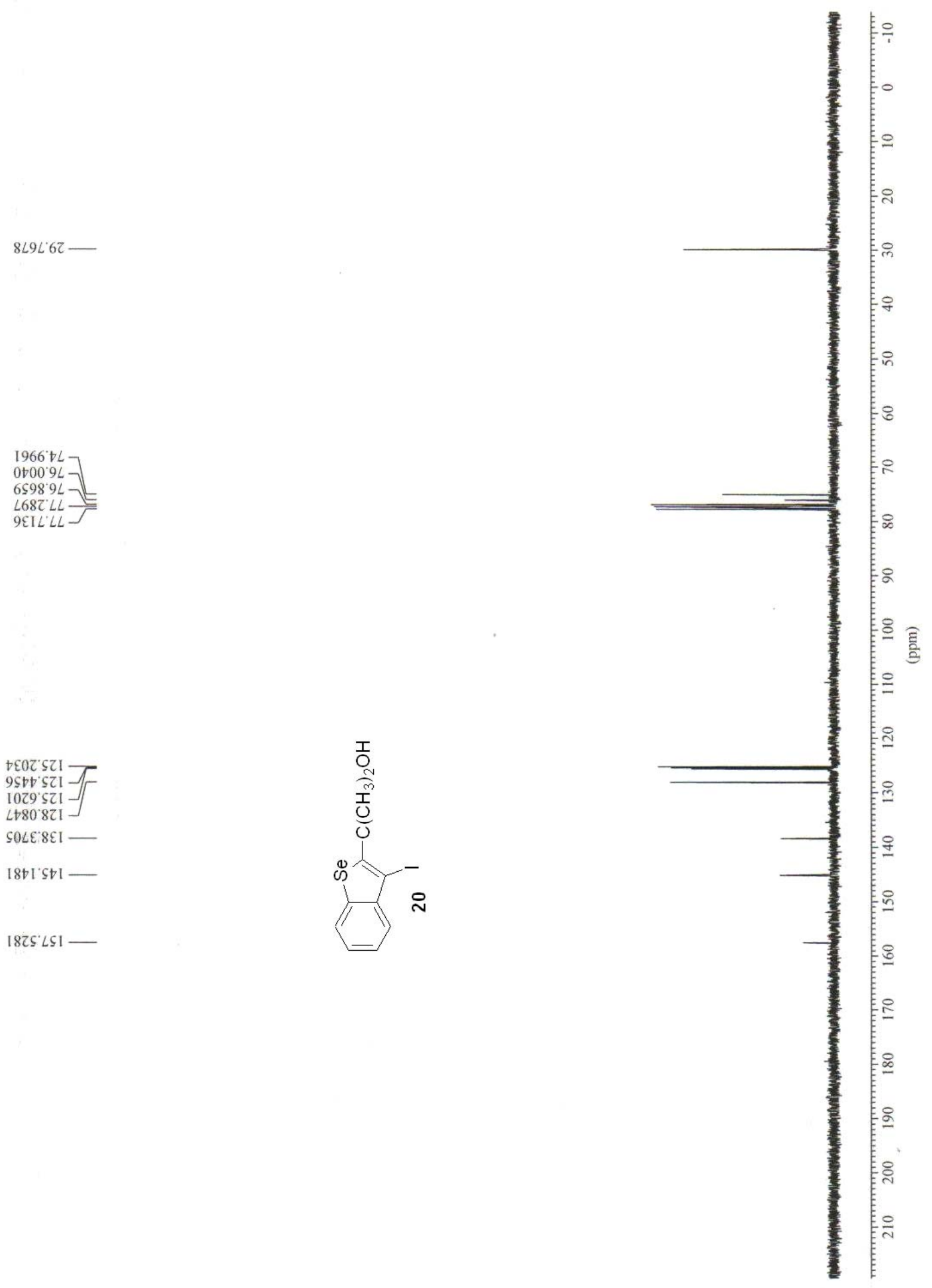


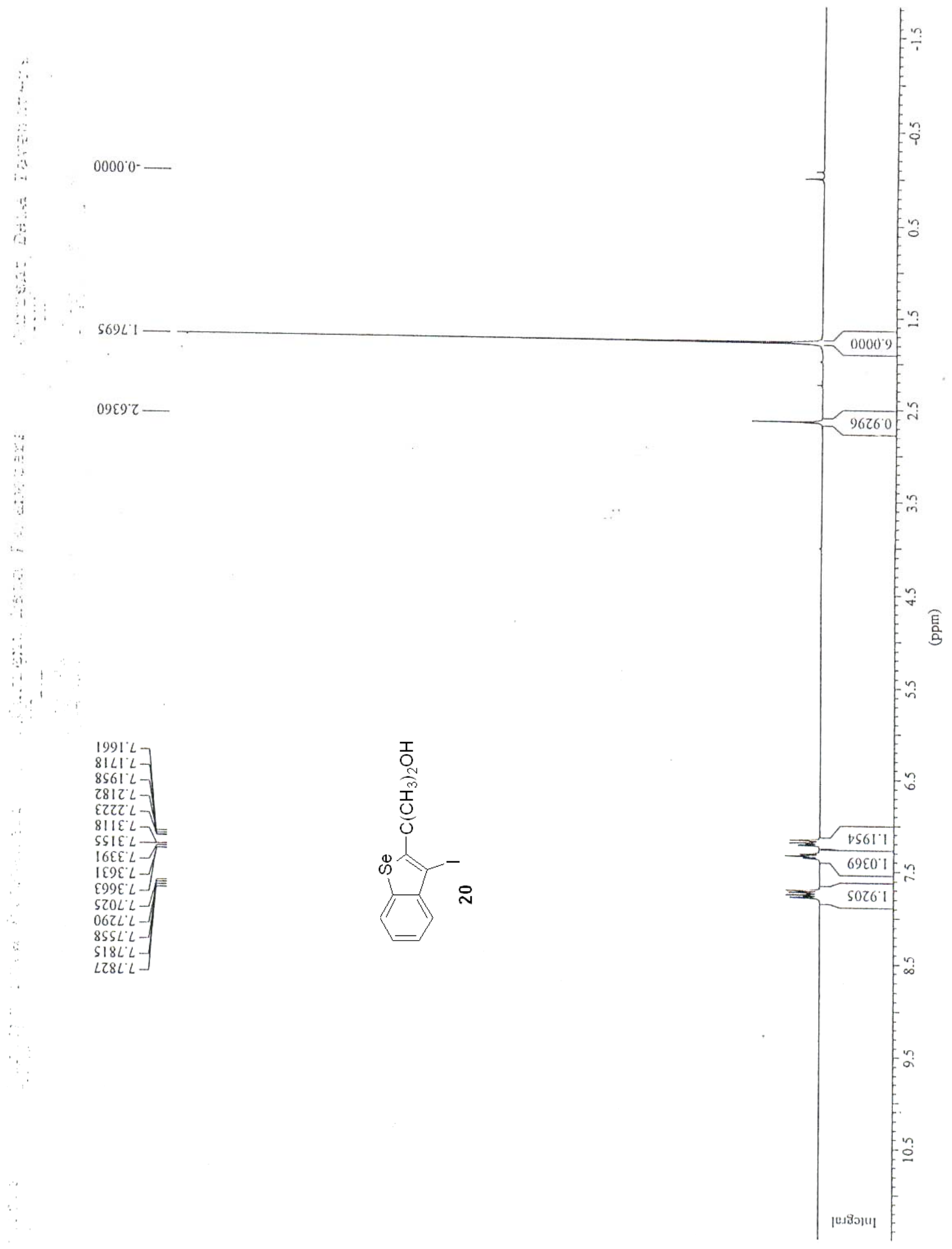




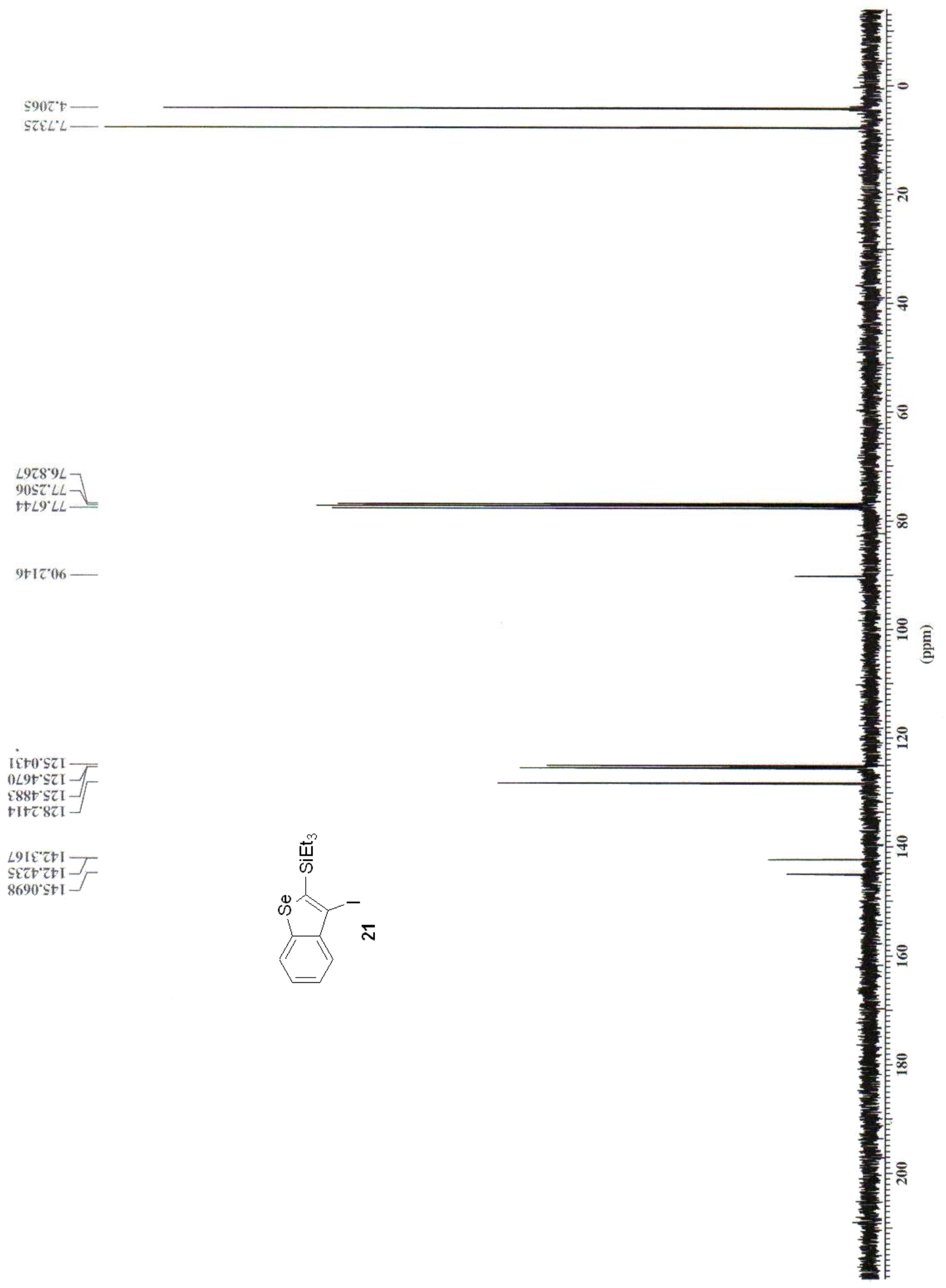




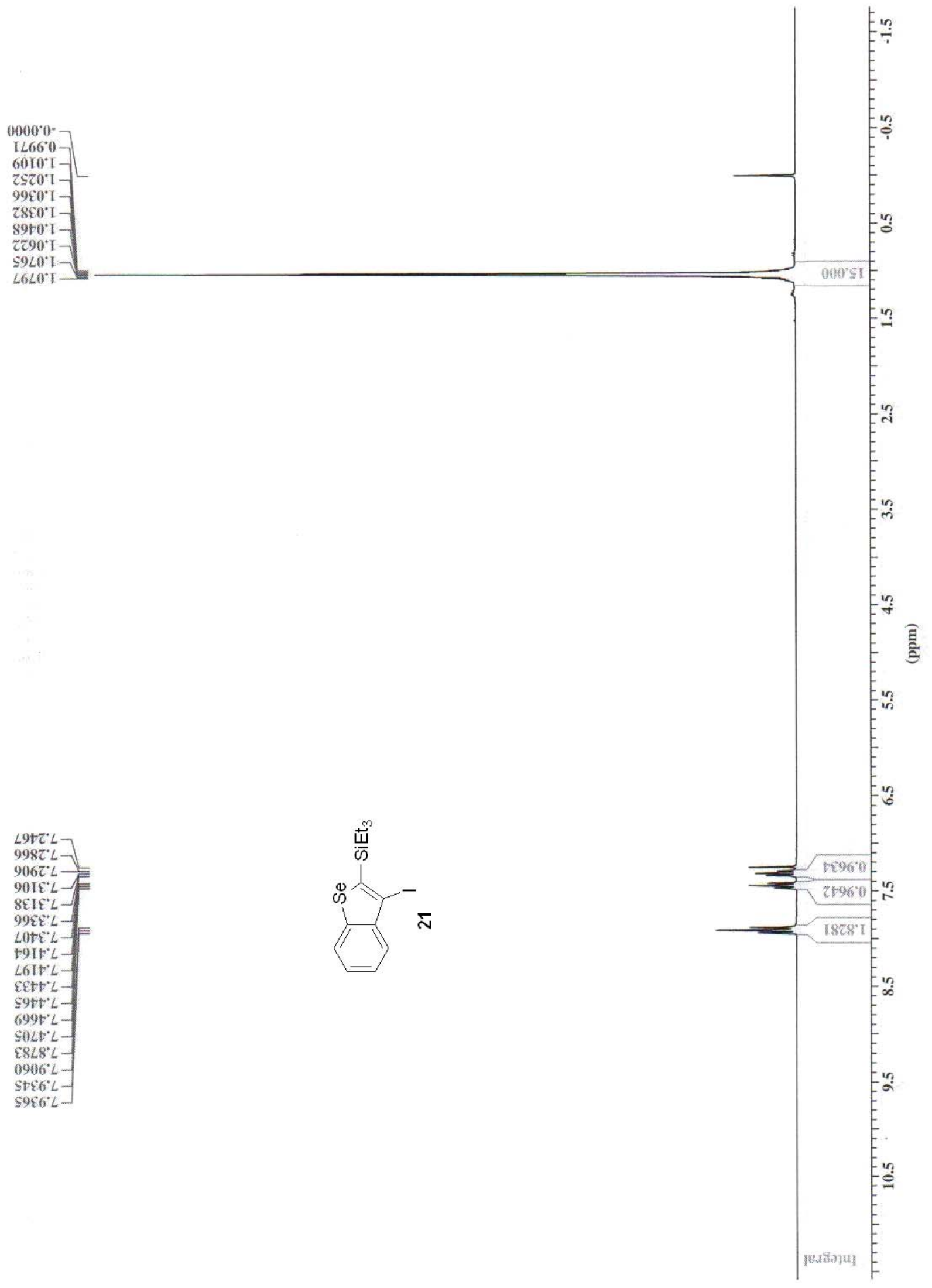




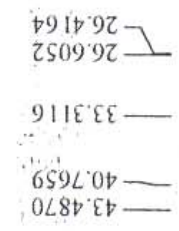

$6 \varepsilon \varepsilon \varsigma^{\circ} \circ 8$

ing

ili

455

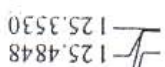

$8780^{\circ} S 21-$
$S 179^{\circ} \mathrm{SZ1} 1$
1096221

909E $6 \varepsilon 1-$

乙LS|E口।-

s818.001-

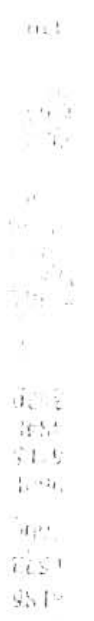

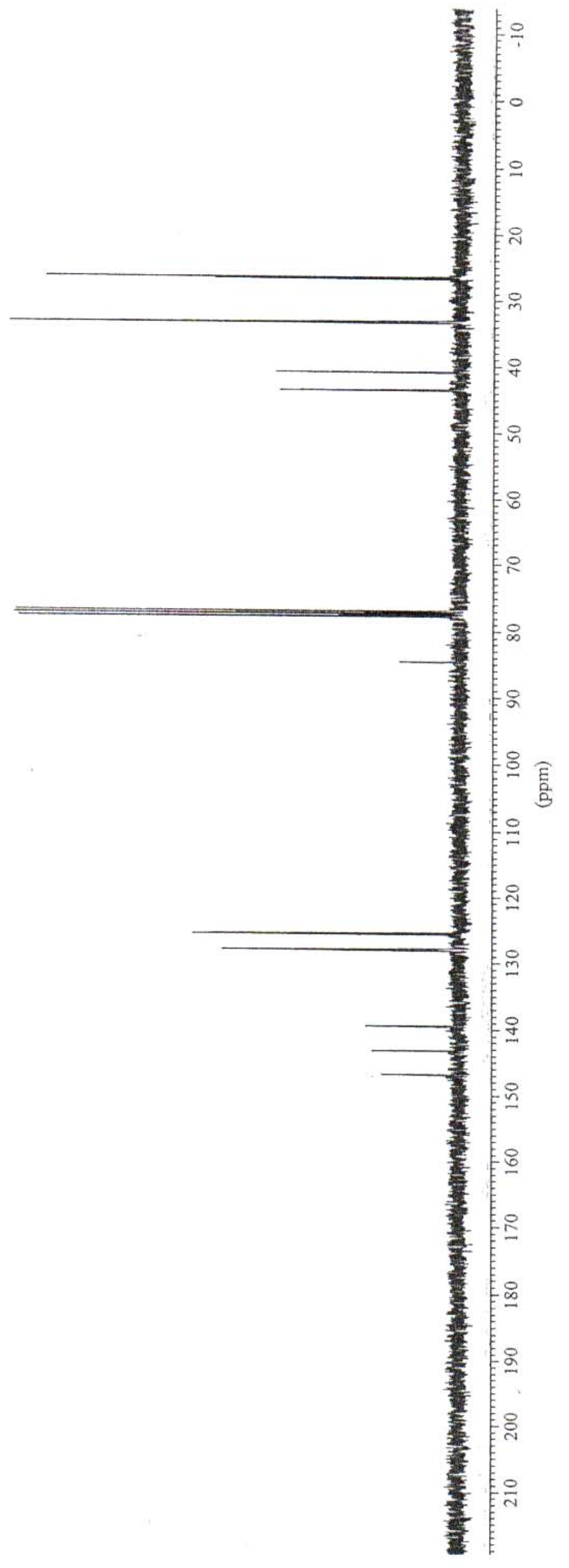




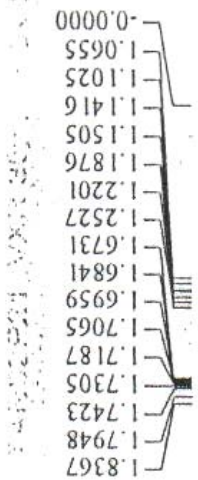

$95162-$

$\therefore$

$\therefore$

$\because$

$98 \mathrm{I}^{\circ} \mathrm{L}$

$\angle D 0 Z L$

E892 L $\mathrm{L}-$

$\angle E L E L-$

$\angle 66 \varepsilon^{\circ} L-$

$1 \mathrm{DC} \mathrm{V}^{\circ} \mathrm{L}-$

OSTLL

$\because \because 2 L L L$

$\angle 78 L^{\circ} \mathrm{L}-$

$0218^{\circ} \mathrm{L}$

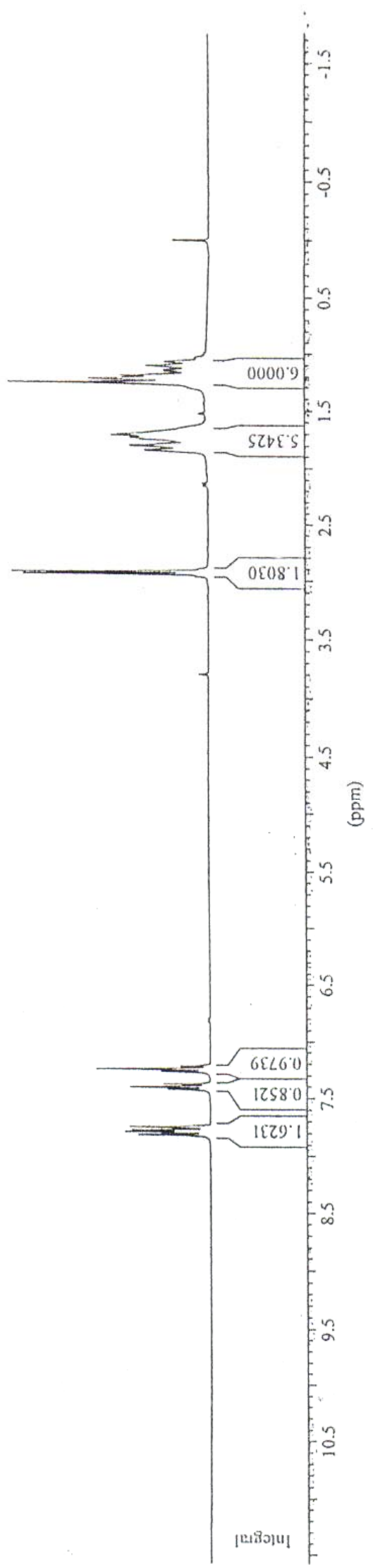



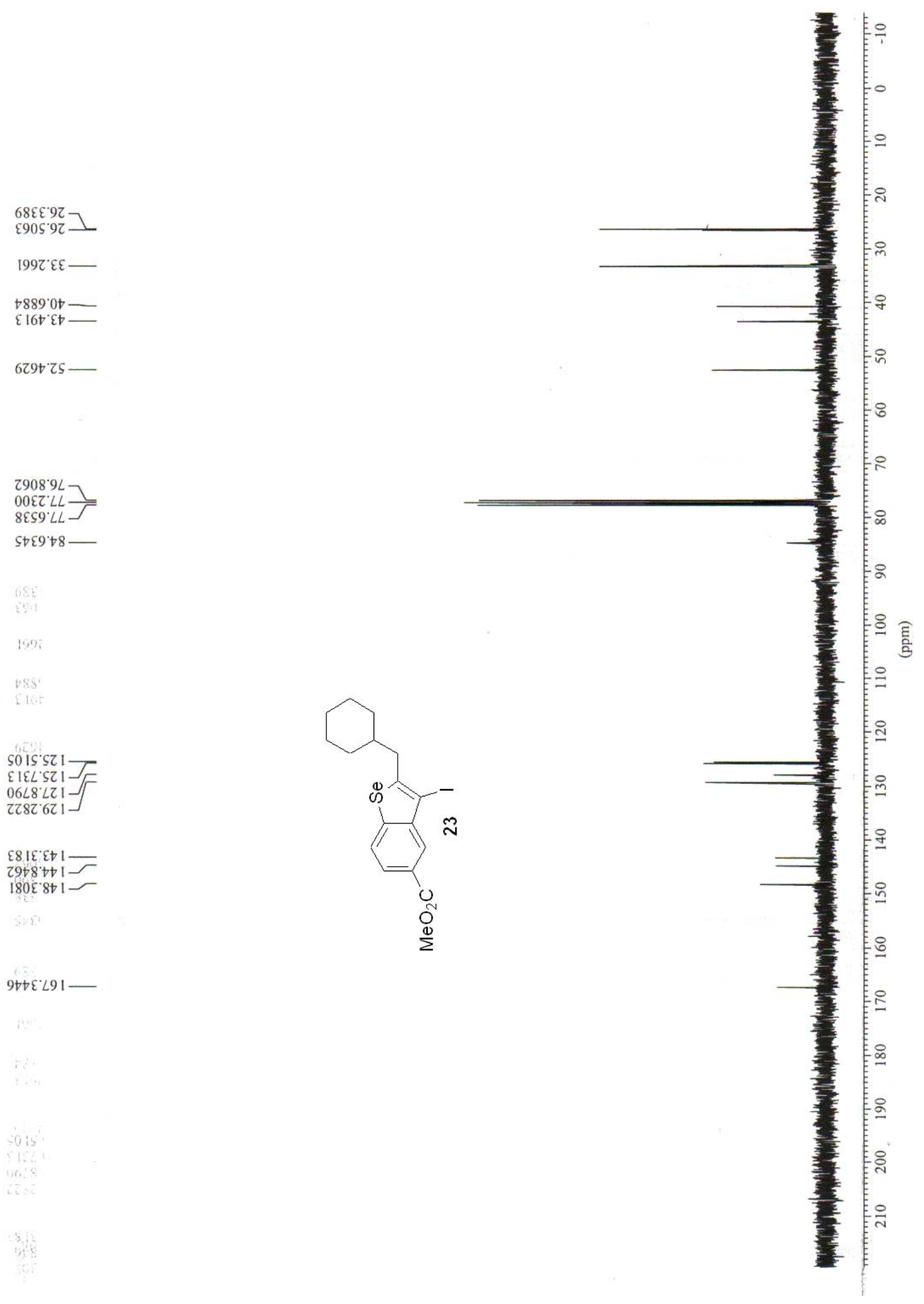

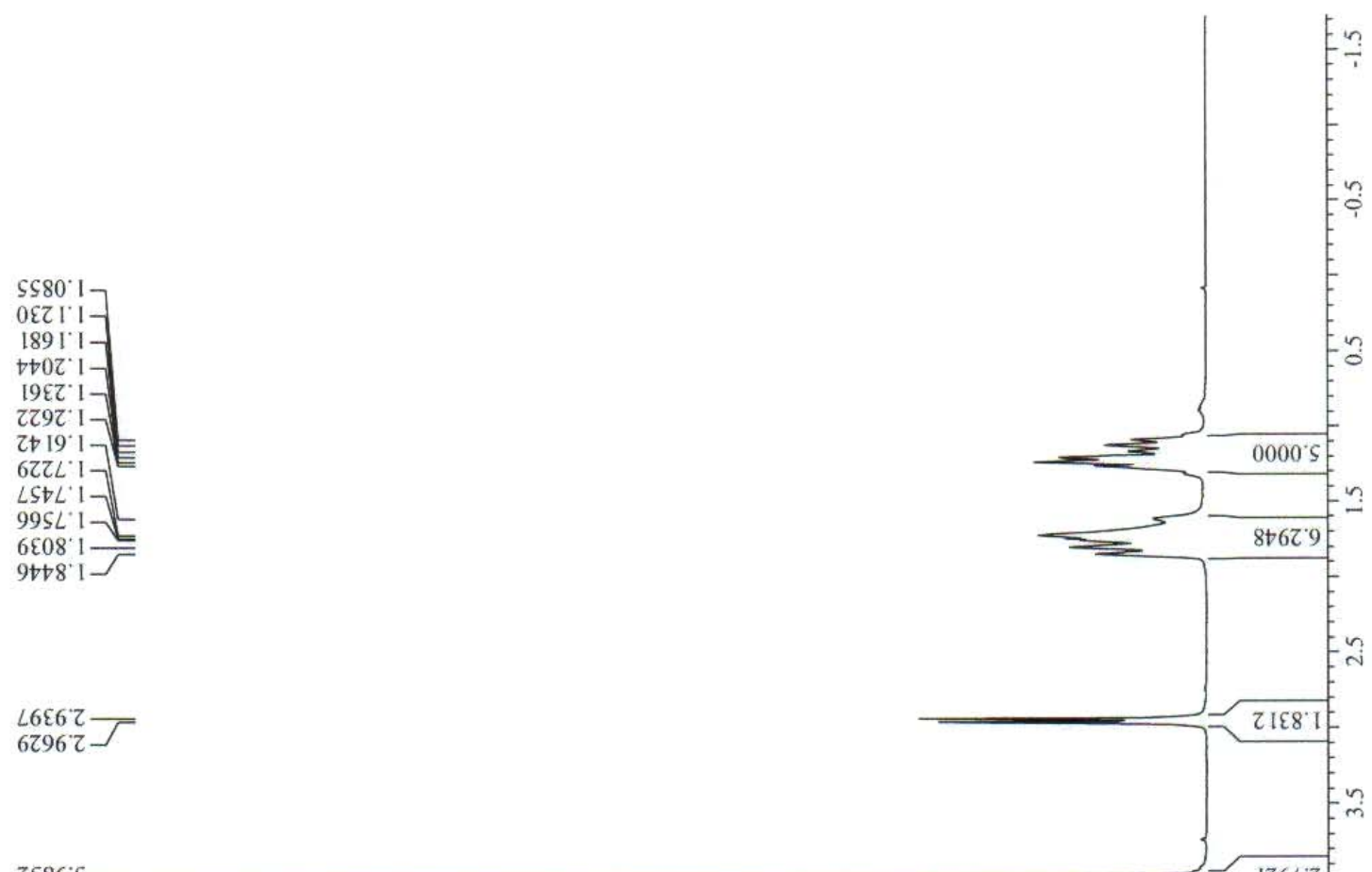

L6E67

$00 \angle Z: L$

$\angle 928^{\circ} \mathrm{L}-\frac{}{\sqrt{F}}$

$S+06^{\circ} \mathrm{L}-$

$9806^{\circ} \mathrm{L}-$

$\angle I E 6^{\circ} \angle-1-$

$869 t^{\circ} 8$
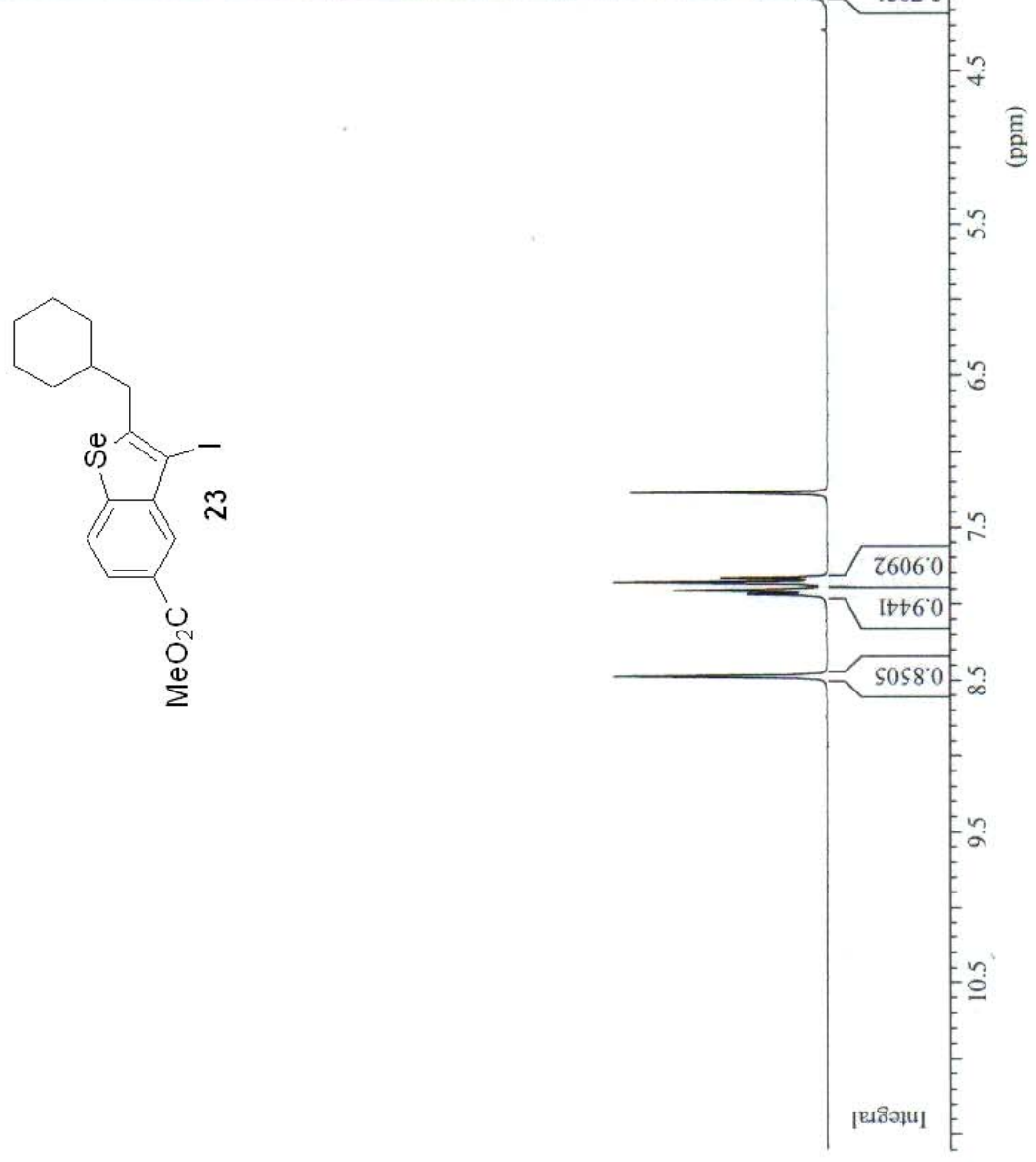

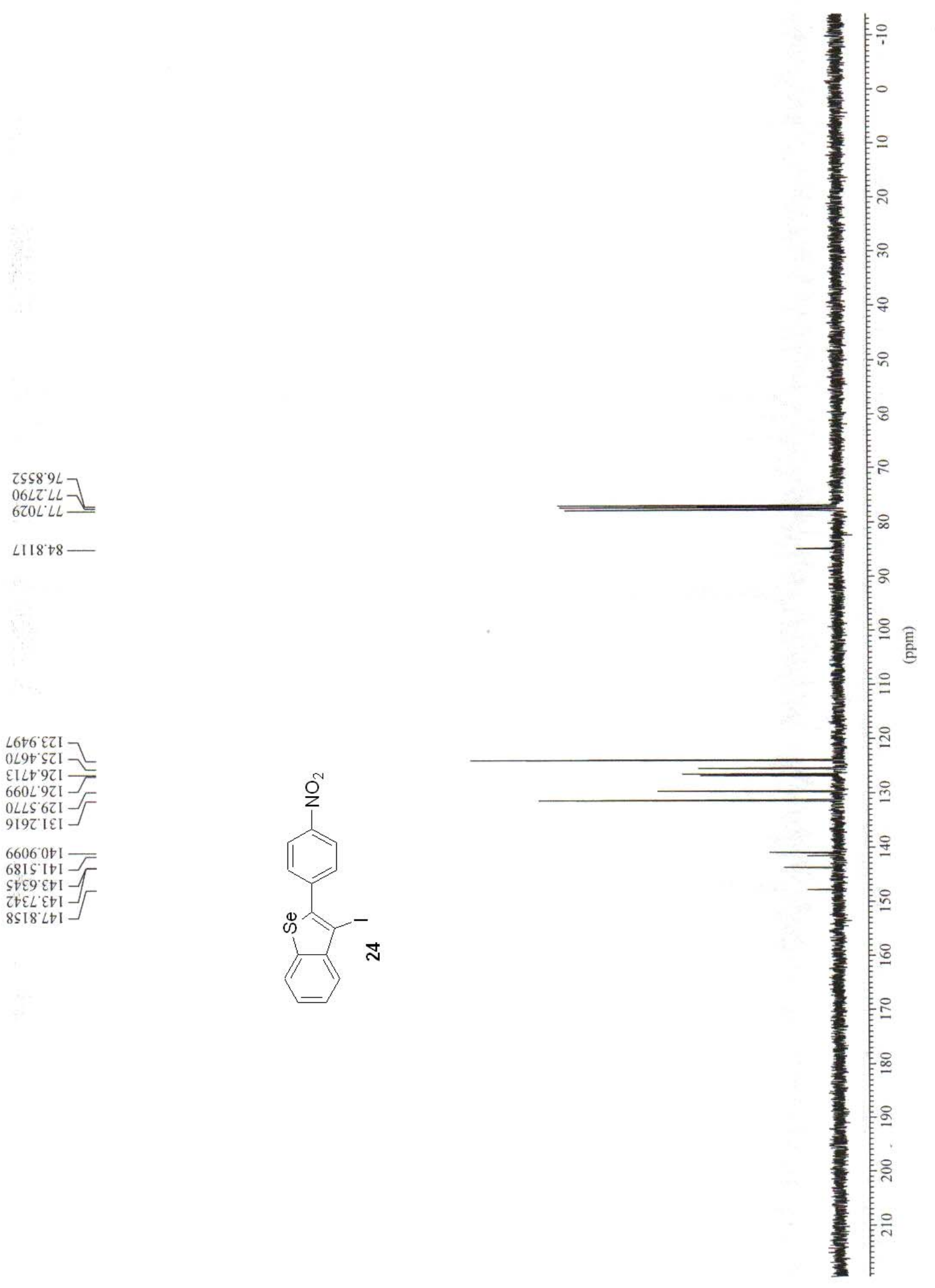
$0000^{\circ} 0^{-}-$
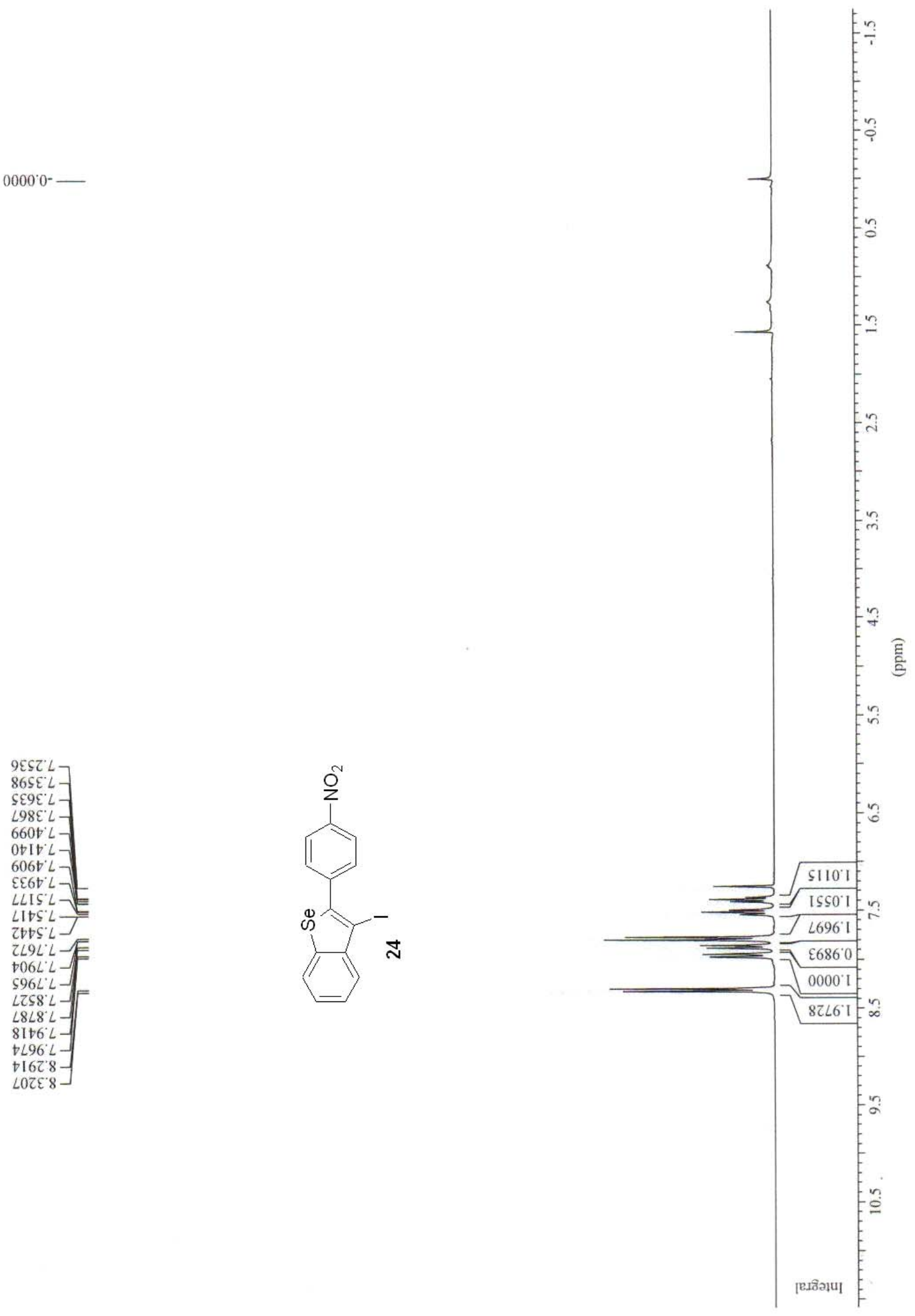


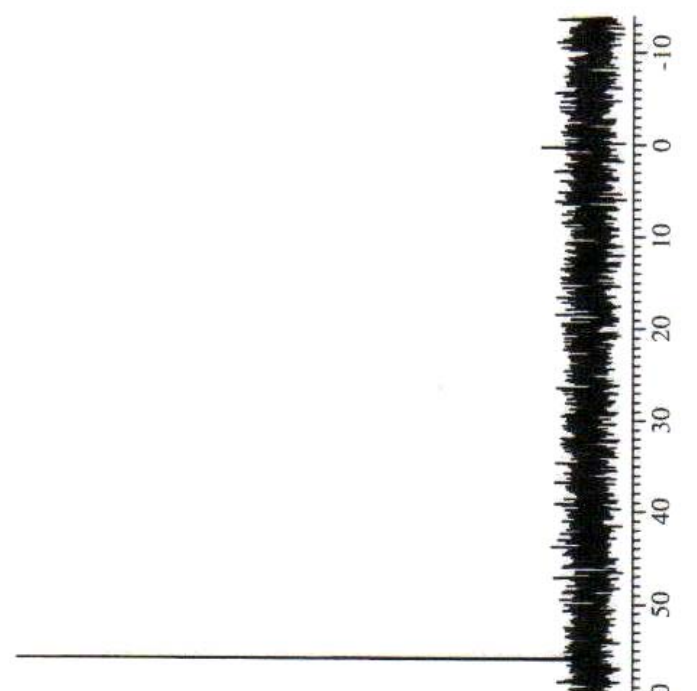

St78 $9 L$

$2269 \mathrm{LL} L$

9400.98

zE29.111-

†509.0ZI -

LS6E'SZI

SOELSCI

LOSL8Z1 $]$ F

ZLLL'OEI

OSऽE $Z E I-$

9Z1Z It I

LLOL'ItI]

0L88.9SI -

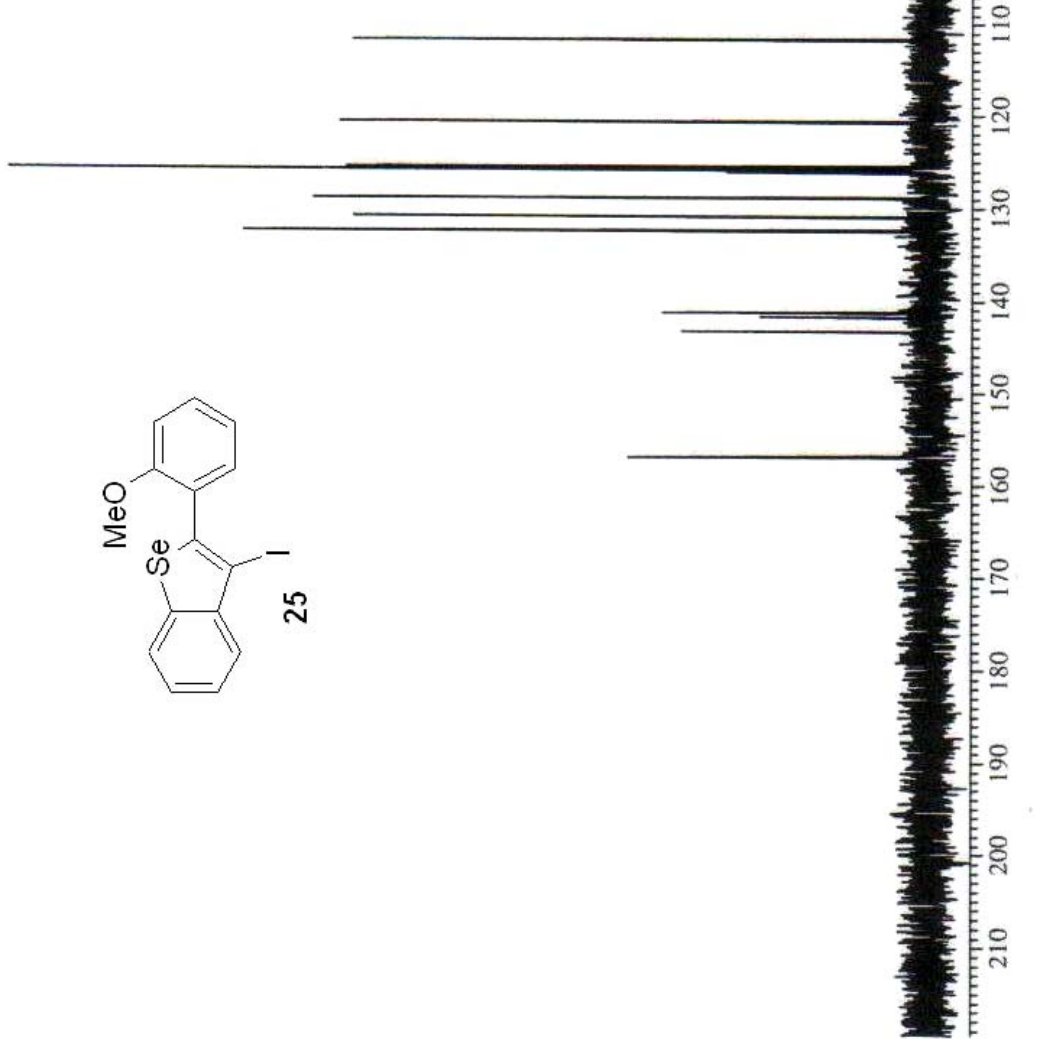



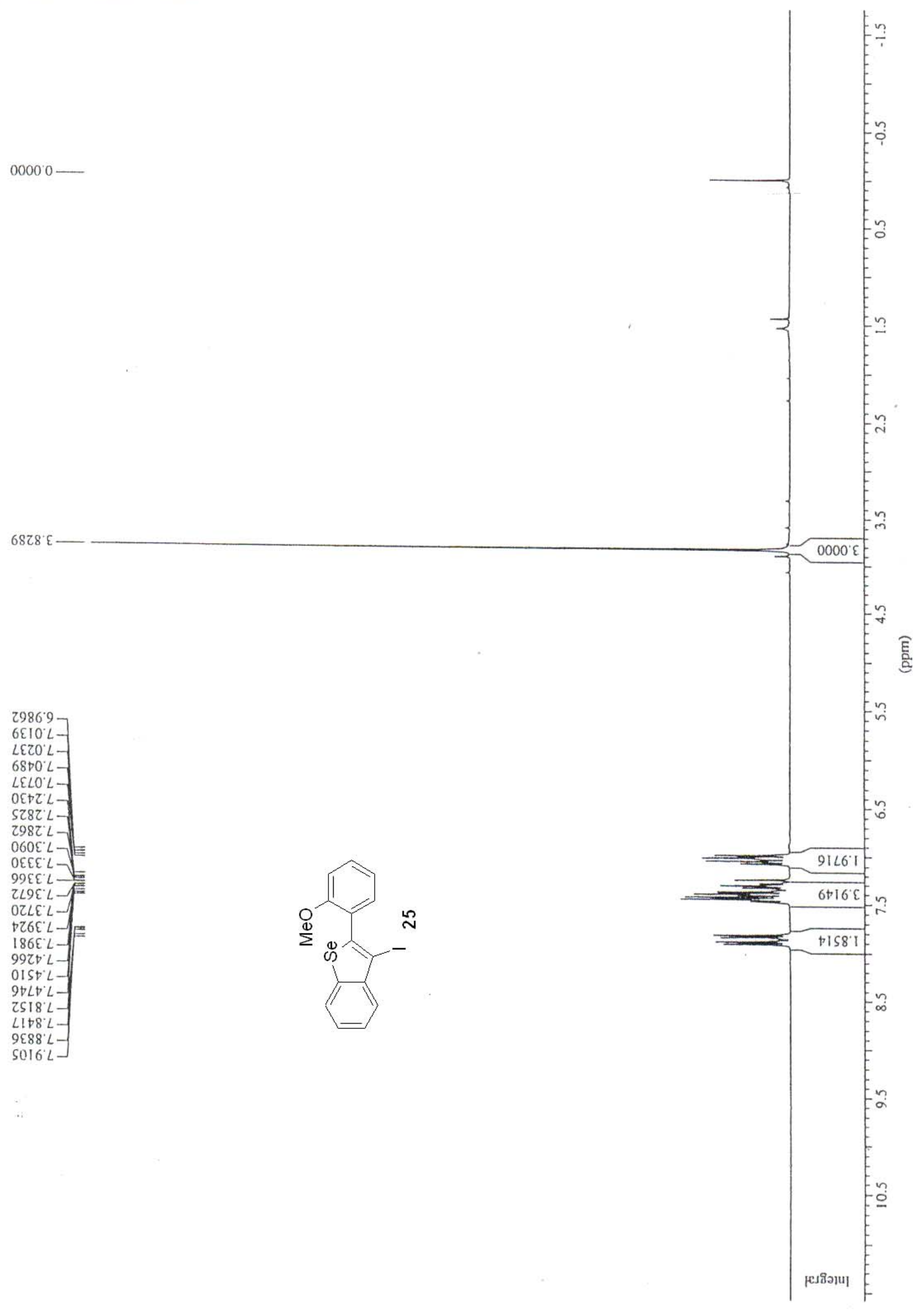


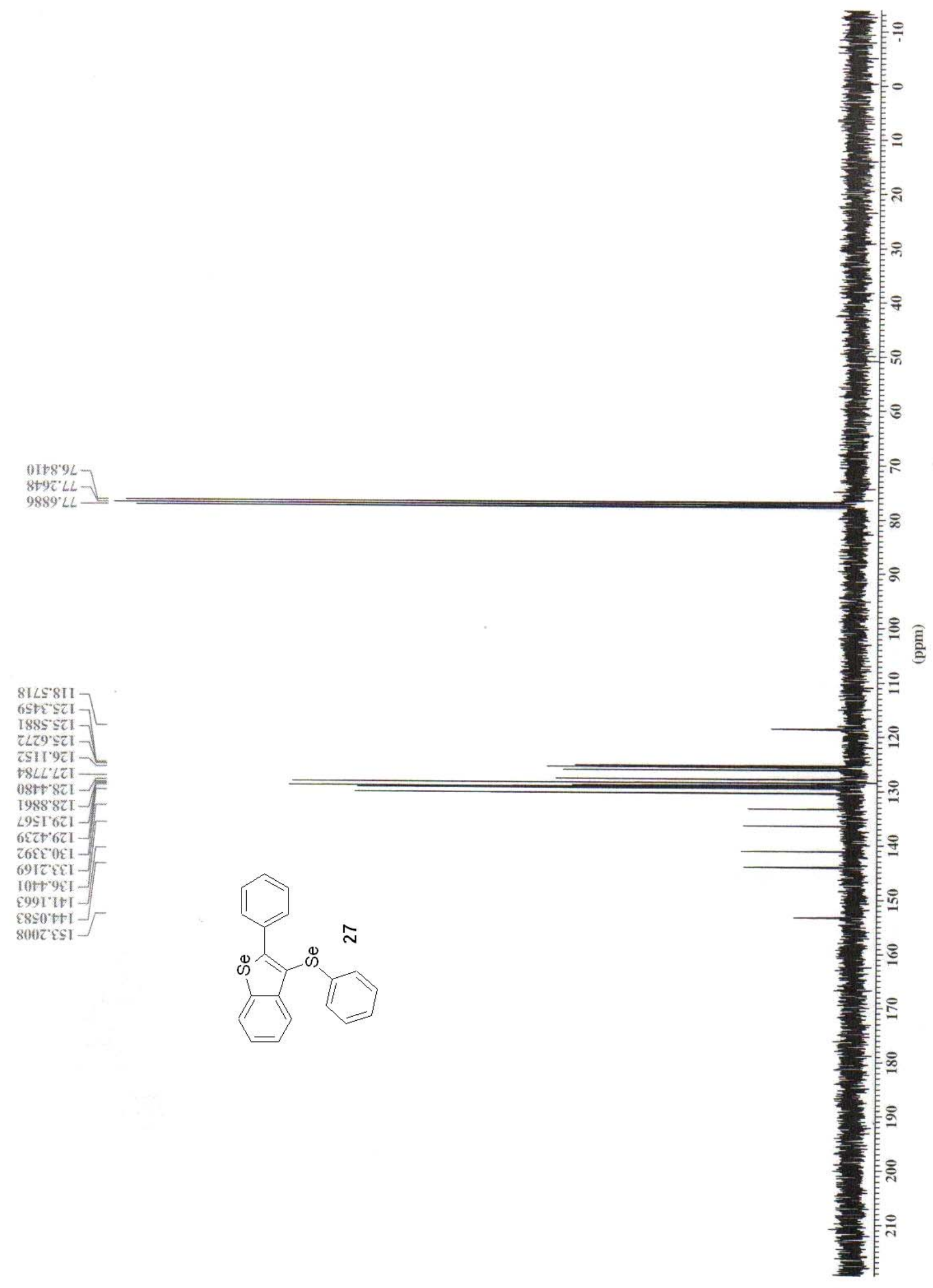


$9000^{\circ} 0-$

รIZS I-

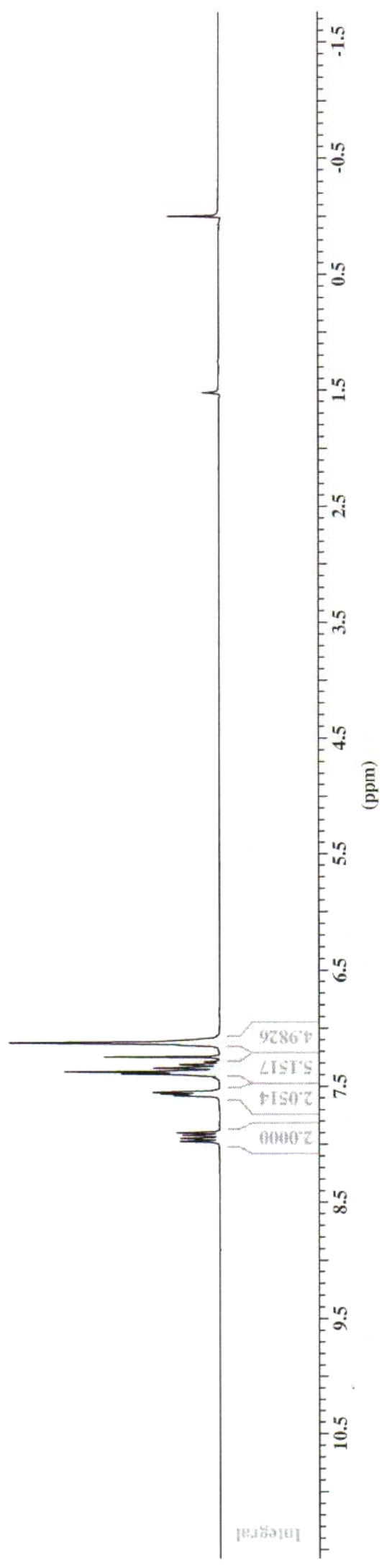




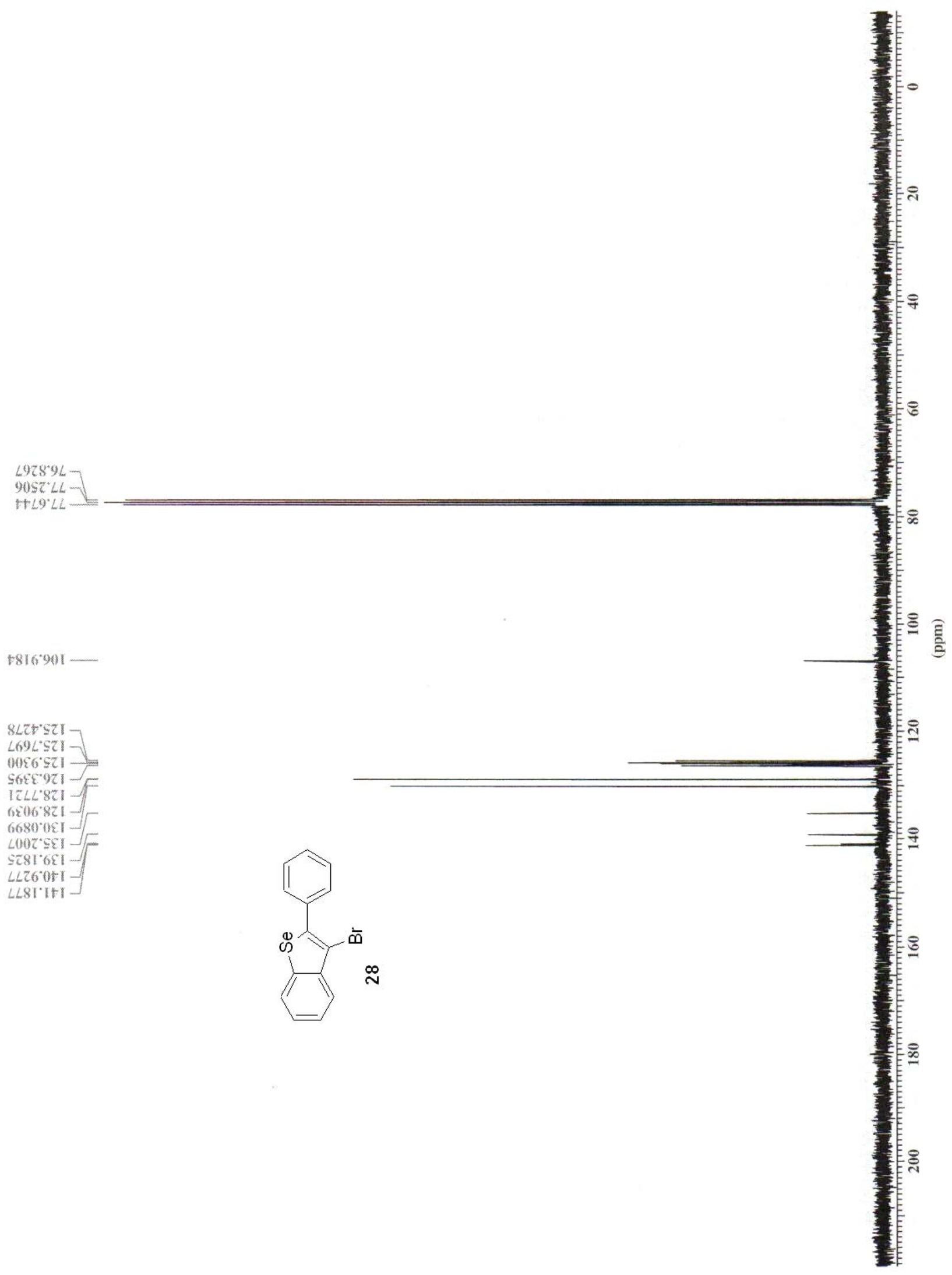




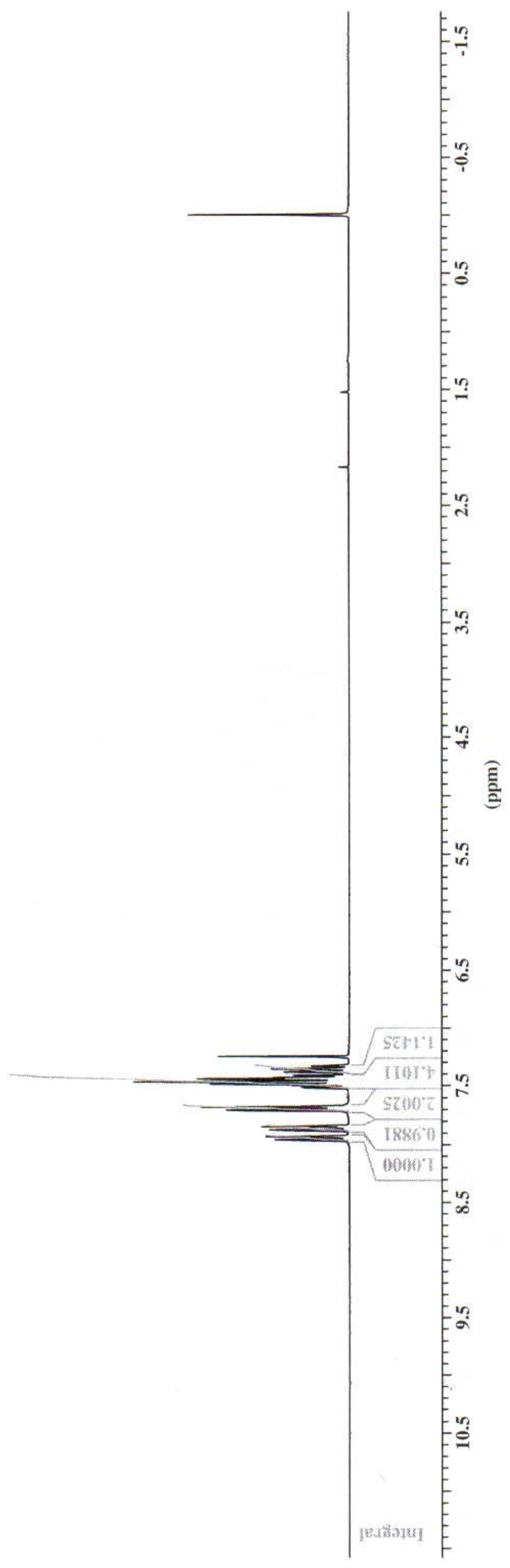



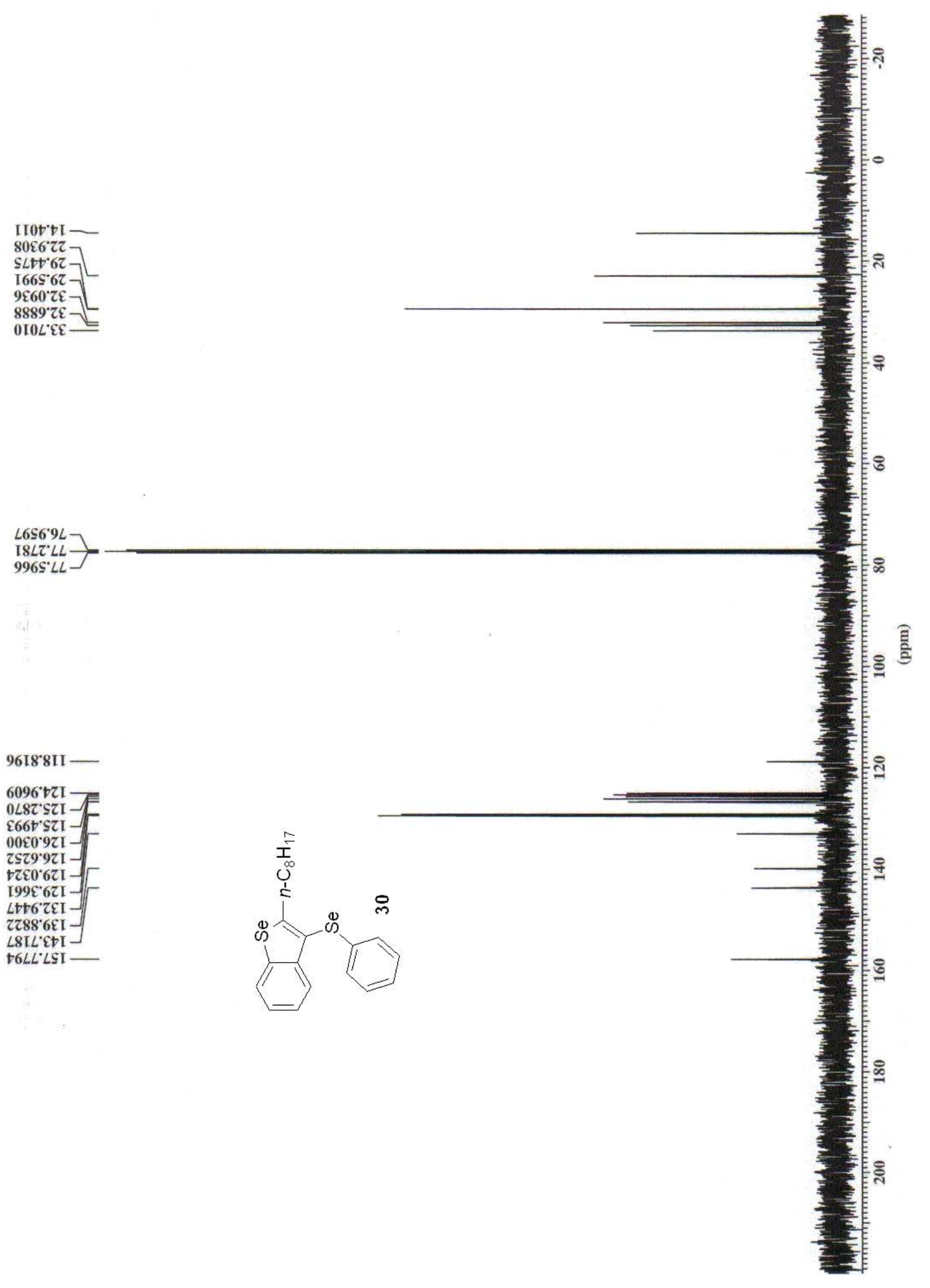

96I8.8II -

$6096^{\circ}+2 \mathrm{I}$

\& $286 t^{\circ} \varsigma 2 I-F$

$00 \varepsilon 0^{\circ} 9 \mathrm{ZI}-$

$+z \varepsilon 0^{\circ} 6 \mathrm{ZI}-$

[998: $62 \mathrm{I}-$

Ltt6 $6 \& \mathrm{I}$ -

$z z 8^{\circ} 6 \varepsilon \mathrm{I}-$

$\angle 8\left[L^{\circ} E t[-\right.$

$\$ 6 L L ' L S I$ 


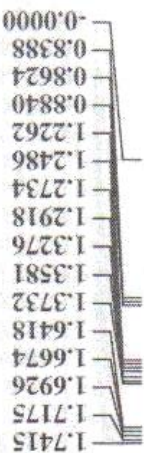

8I8T'

$8 \angle 0{ }^{\circ} \mathcal{E}$

ตัยน'

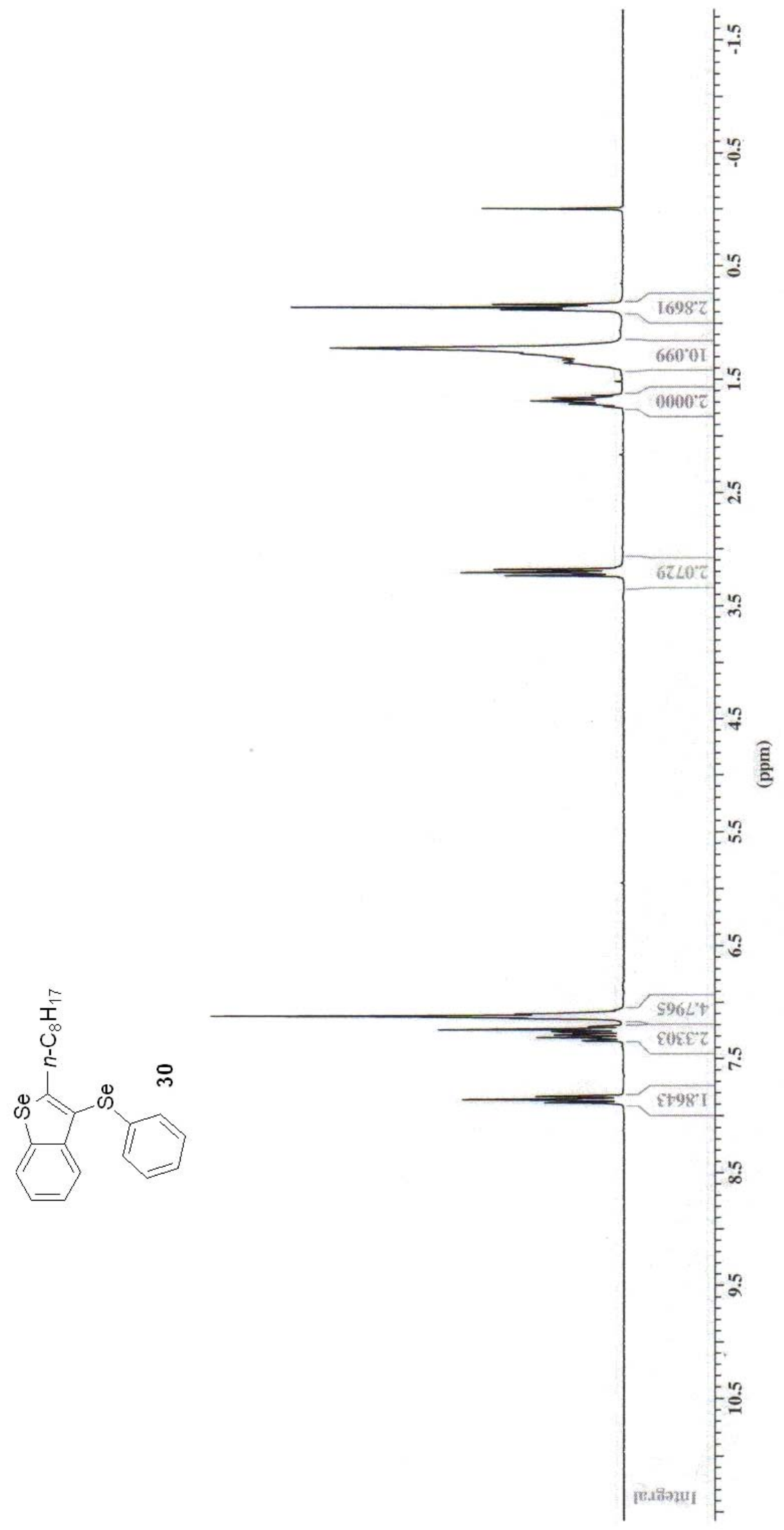


S09E.t -

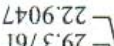

zott 62$]$

$6 \mathrm{I} \angle \mathrm{S} 6 \mathrm{Z}$

SLZt IE

$\varsigma \varepsilon 60 Z \mathcal{Z} Z$

$\& 29 \circ 201-$

9S8 ['SZI-

$00 \mathrm{It} \ \mathrm{I} \mathrm{I}$

$8 \varepsilon \angle \varsigma$ ऽZ I

S IZ0 $8 \mathrm{EI}$

$\rightarrow Z \varepsilon S^{\circ} 0 t 1-$

I6ES $t+1$
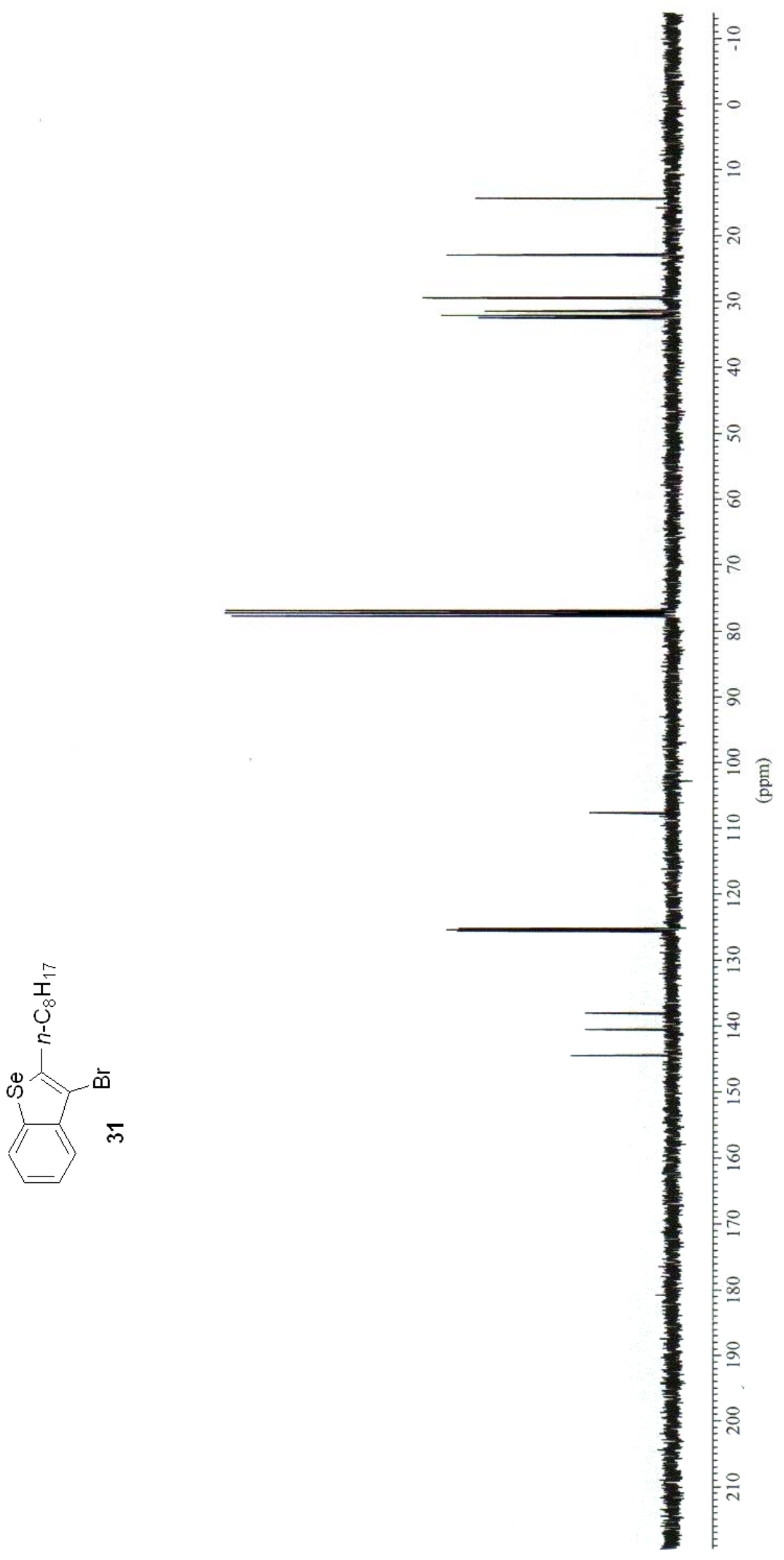


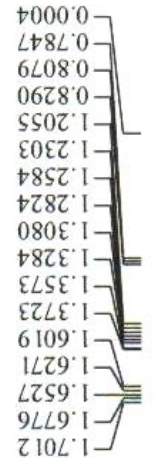

$6 \angle 88^{\circ} Z-$
$9 \mathcal{E} 16^{\circ} Z-7$

$88 \mathcal{E} 6 \mathrm{Z}$

$9+\angle I^{\circ}$

$8 L 6 I L-$

Z0Z2: L

$\varepsilon+z z^{\circ} L-$

SSIE $L-$

I6 IE $L$

$\varepsilon Z \downarrow \varepsilon^{\prime} L-$

$\angle 99 \varepsilon^{\circ} L$

Z69E $L$

$0 L O L L$

$8 \varepsilon E L^{\prime} L$

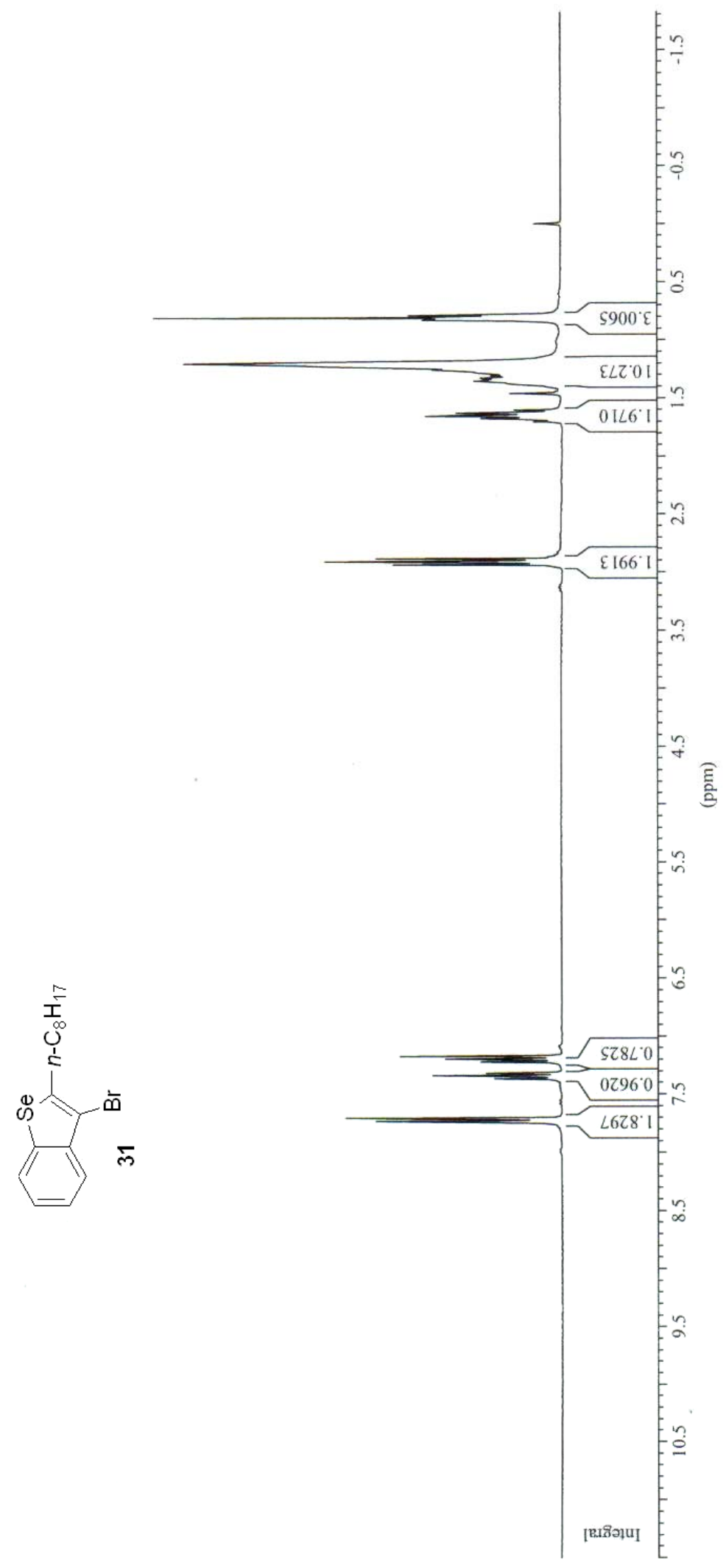


$8856 \cdot 17$

LSLO

$\$ 826$.

OLOT'TE-

†LE⿰冫) $L$

ZI9Z: $\angle L$

$1589^{\circ} \angle L L$

$\angle H+L^{\circ} 9 \mathrm{HI}$

$9960^{\circ} \mathrm{SI}$

$1828 \mathrm{sI}$
$1696 \mathrm{~g} 1$

$\angle L 0[-\angle z I] F$

$0 \$ 8 \pi^{\circ} 6 \tau 1$

$8200^{\circ} 621$

8zร8. IEI -

ऽostegl -

$89+0$ t $E I$ -

tt+0.0t I

IS $\angle 8^{\circ} C T I$

tIEะ') I

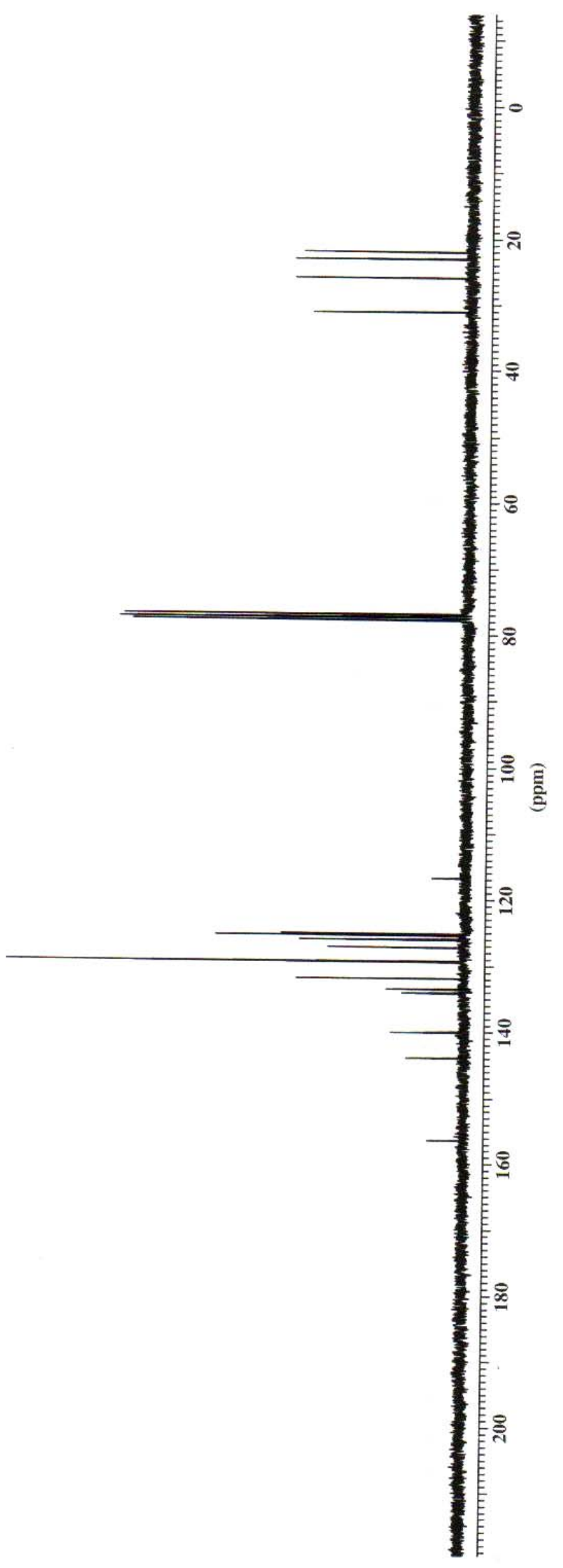



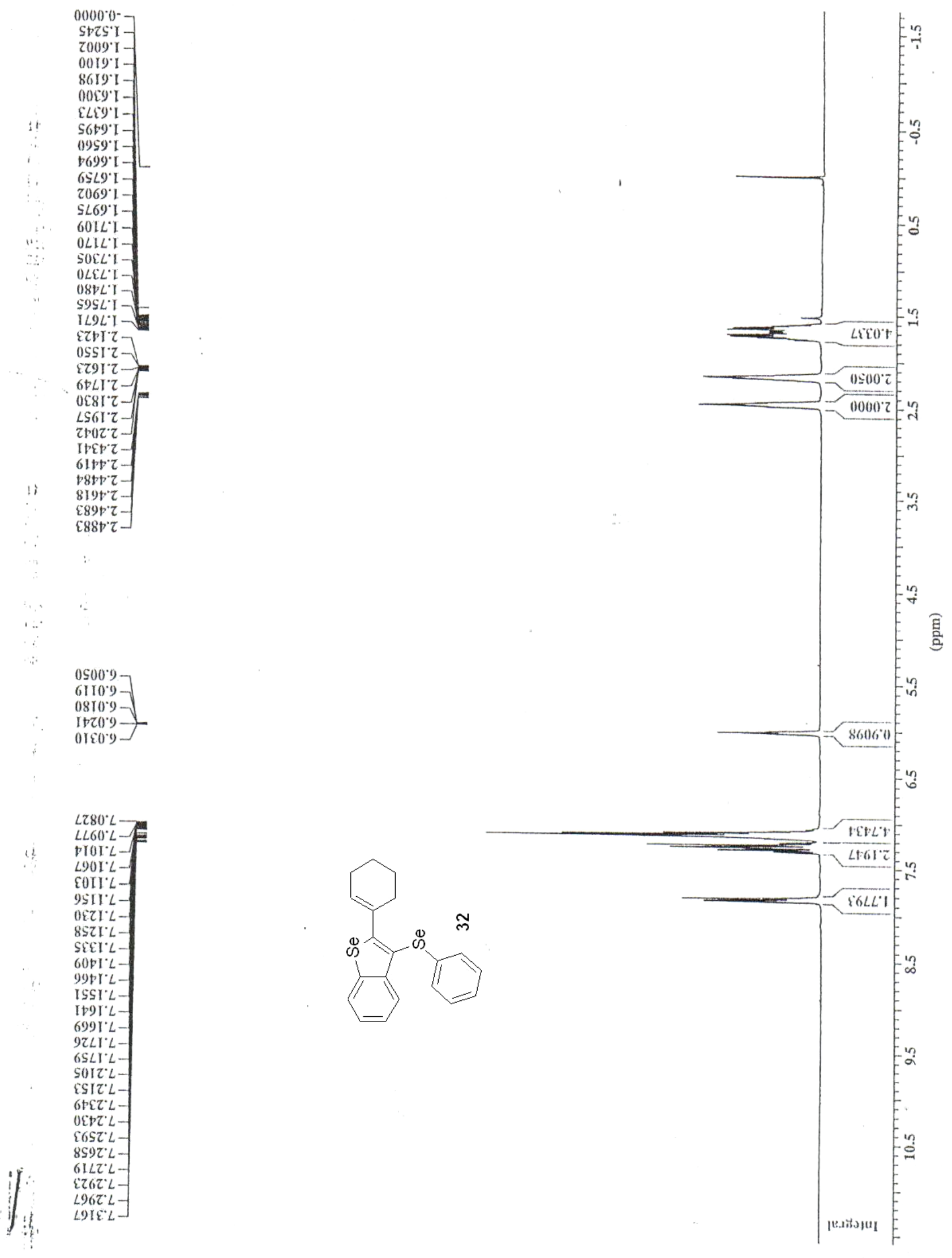
$89 \varepsilon 6^{\circ} \mathrm{Iz}$

$9 \mathrm{IS0} 0^{\circ} \mathrm{E}$

$\$ 896^{\circ} \mathrm{Z} Z$

†00E $0 \mathcal{E}-$

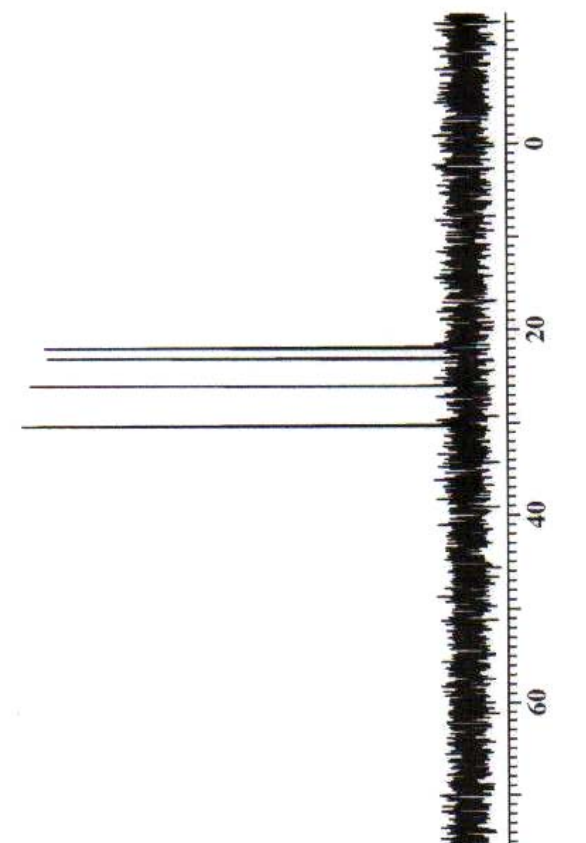

2908.92

$8599^{\circ} \angle L$

$9900^{\circ} \mathrm{s}_{0} \mathrm{I}$
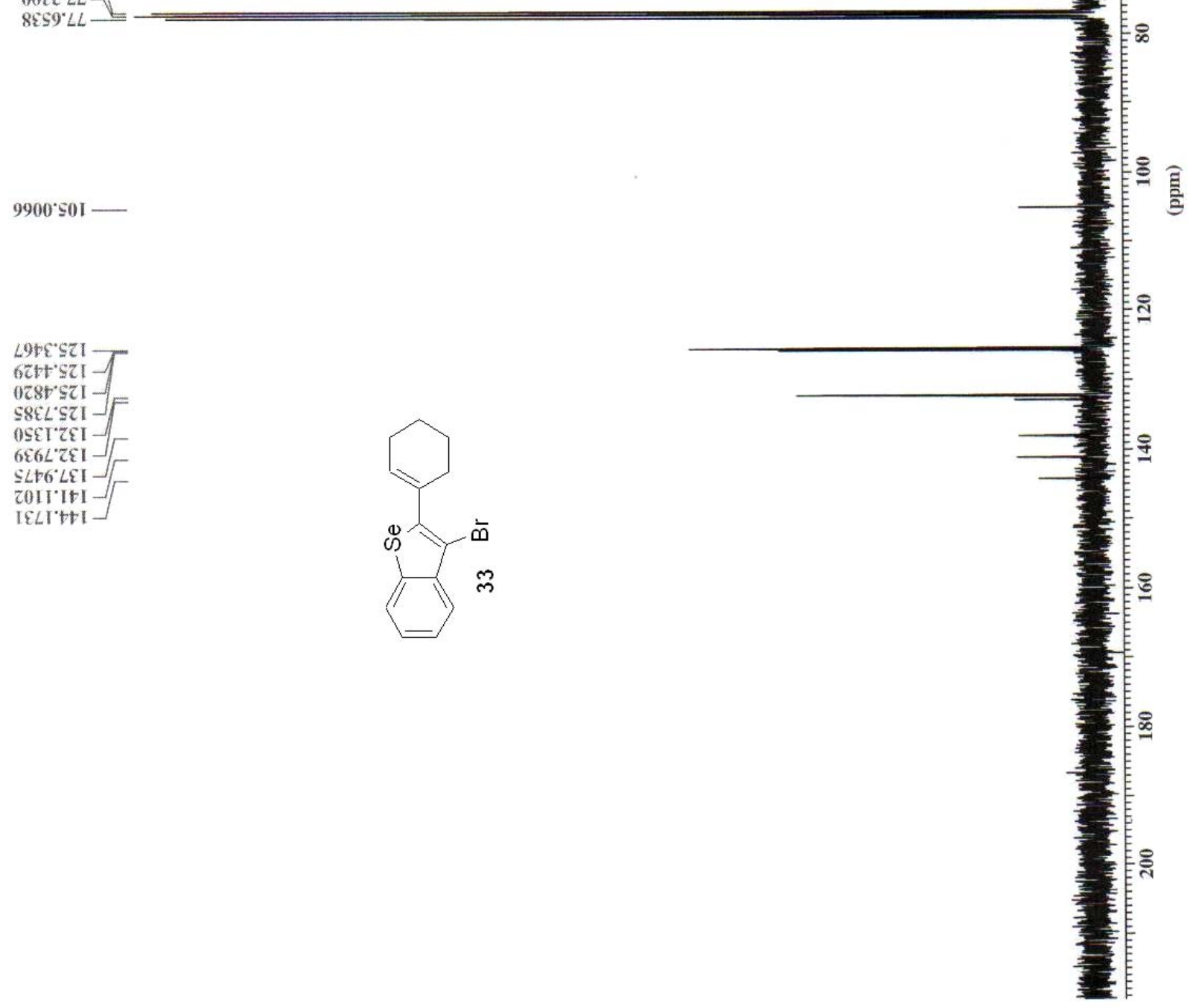

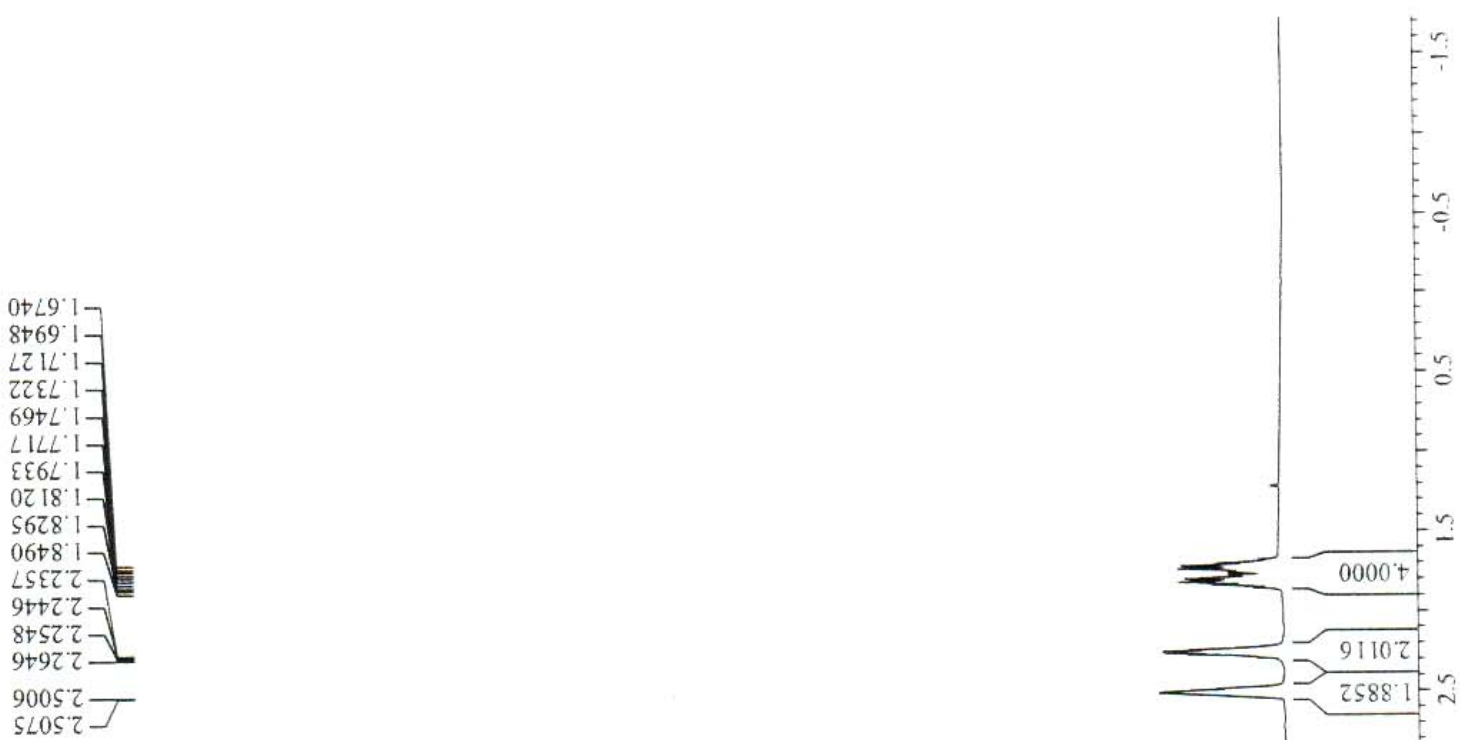

sLos'

60529

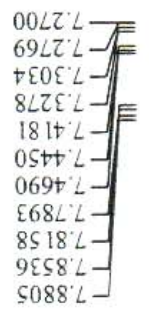

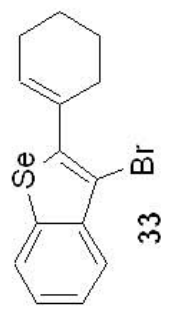

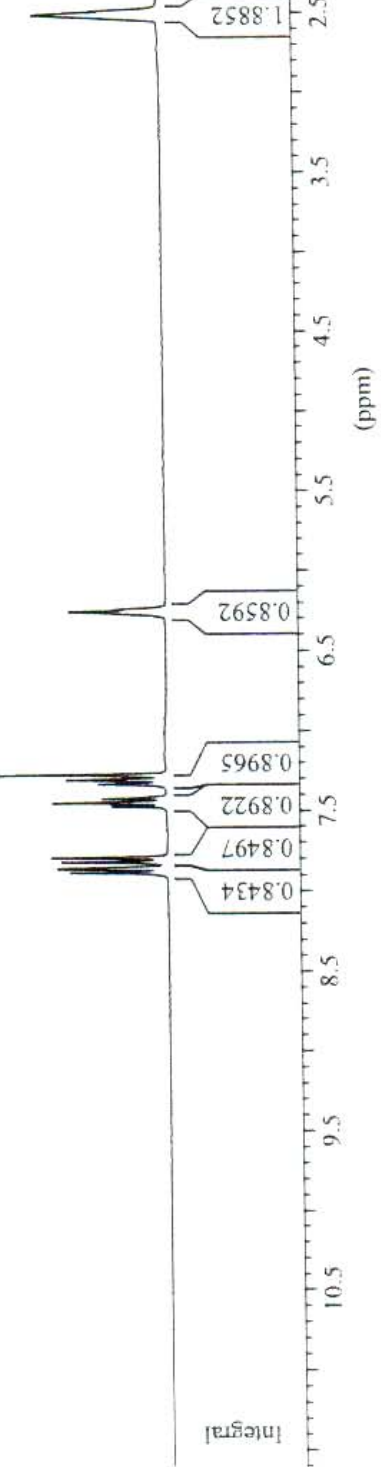


$86 t \varepsilon+1$

EE88 ZZ -

$9 \angle \forall E 6 Z$

E06E $6 \mathrm{Z}$

$t$ I IS. 62 -

$8181^{\circ} 1 \varepsilon$

$98907 \varepsilon$

2866 $2 \varepsilon$

E0E8 $9 L$

It $S Z^{\circ} L L$

6LL9LL

$0 \succ z z \circ z^{\circ}$

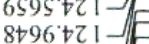

Z8ESSZI

$\angle \angle O S^{\circ} \angle Z I-$

$\forall+0<L^{\circ} 8 \mathrm{Cl}$

$\angle L 9 E \mathcal{E}^{\circ} 0 \mathcal{1} 1-$

SL98 $9 E \mathrm{E} I-[-$

$\angle \angle Z 9^{\circ} 6 E \mathrm{I}$ I-

8zStict

$66\left[9^{\circ} \mathrm{LDI}-\right.$

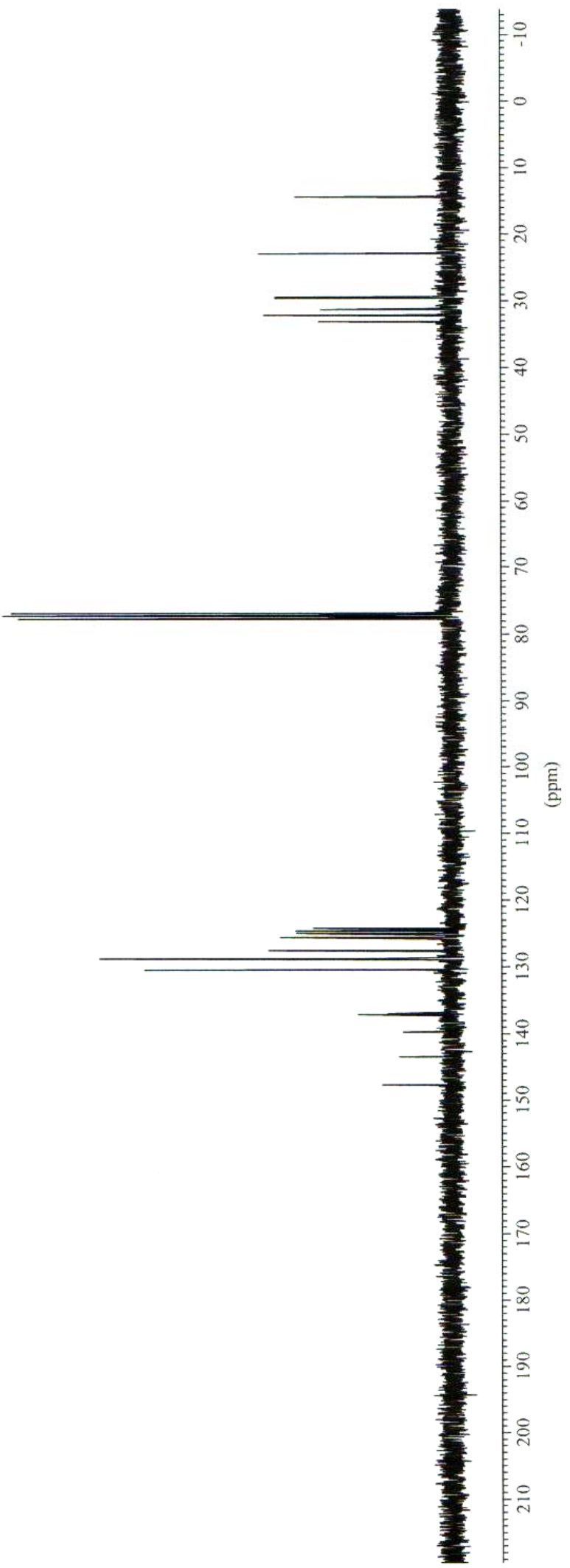



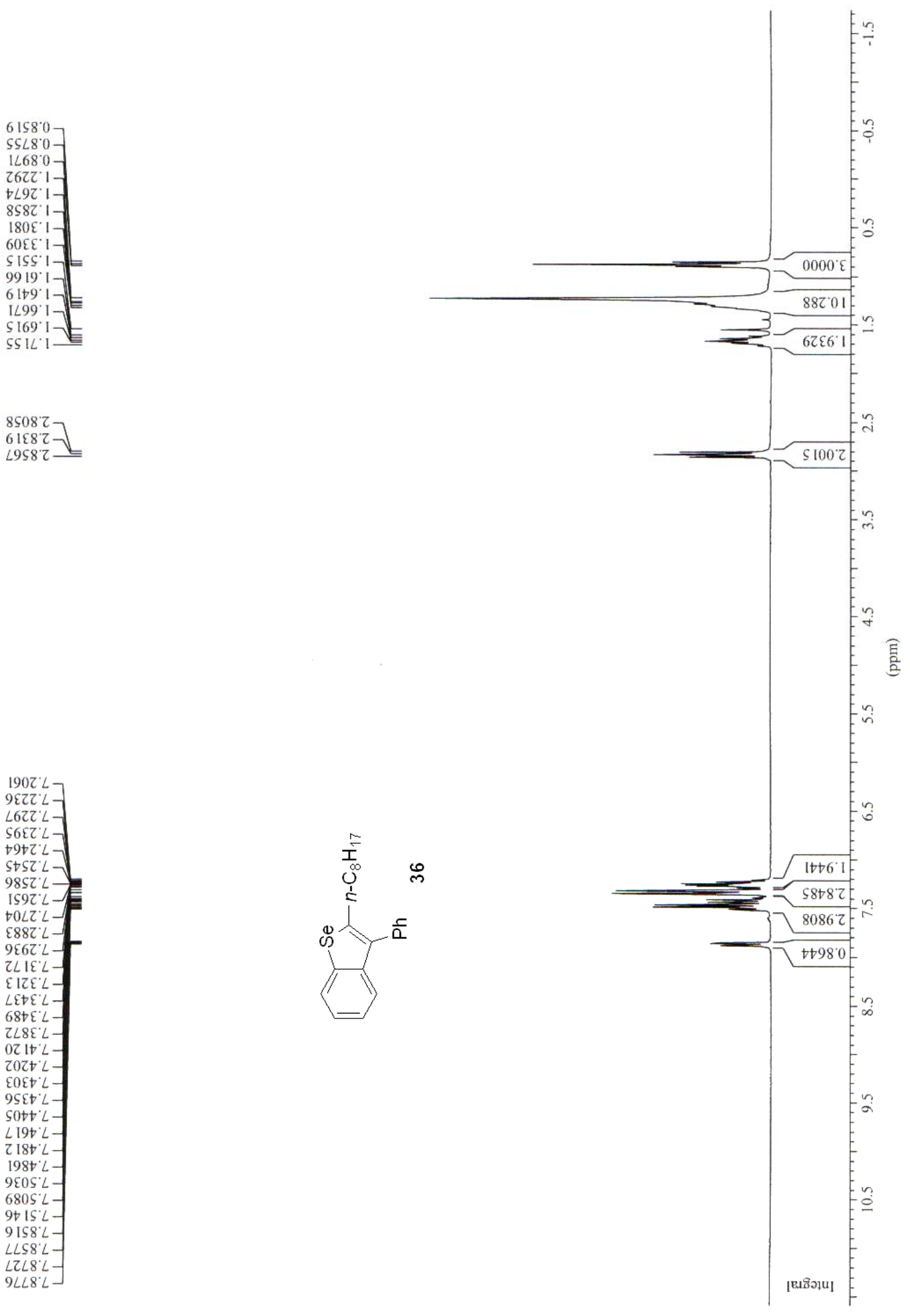

$8508^{\circ} \mathrm{Z}$

$6188^{\circ} 7-$

$1902 \mathrm{~L}$

$9822 L$

S6EZ L

$\nabla 9+2: L$

$S D S Z L$

$9852 \mathrm{~L}$

IS92 $L-$

$8882^{\circ} \mathrm{L}-$

ZLIE'L

¿IZE'L

Let

0210

zozt

EOEt $L$

9SE ${ }^{\circ}$

$198 t^{\circ} \mathrm{L}$

$9 \varepsilon 0 S^{\circ} L$

60s

$9 t I S L$

9 IS $8^{\circ} \mathrm{L}$

$\angle L S 8^{\circ} L$

$\angle Z \angle 8^{\circ} L$

$9 L \angle 8^{\circ} L$ 


$$
i=\neq
$$




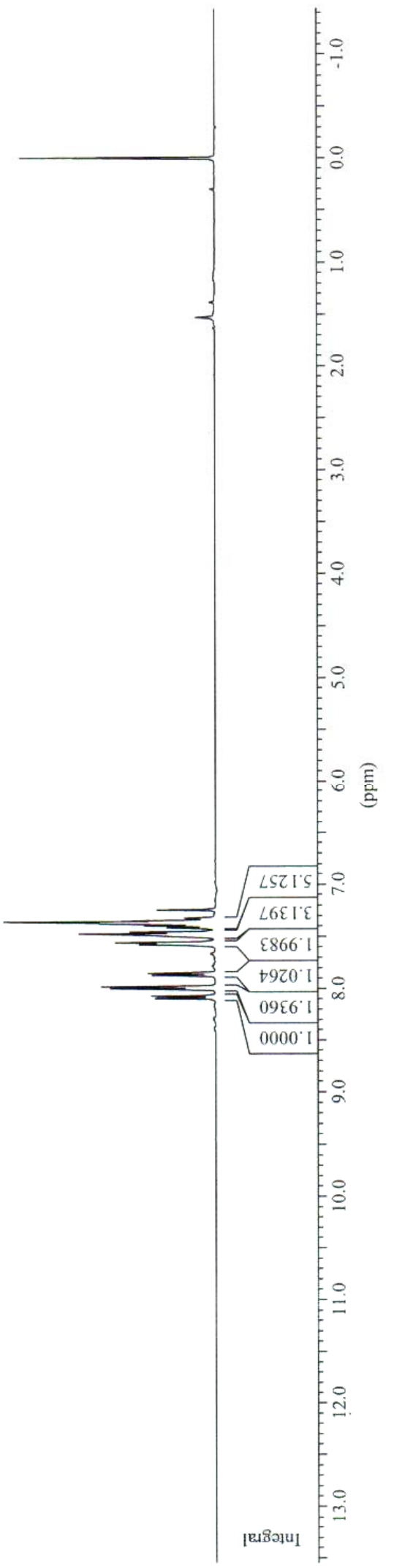

